Supporting Information

\title{
Concise Synthesis of Isocoumarins through Rh-Catalyzed Direct Vinylene Annulation: Scope and Mechanistic Insight
}

\author{
Gen Mihara, ${ }^{\dagger, \S}$ Koushik Ghosh, ${ }^{\dagger, \S}$ Yuji Nishii, ${ }^{* \dagger}$ and Masahiro Miura*,† \\ $\dagger$ Department of Applied Chemistry, Graduate School of Engineering, Osaka University, Suita, Osaka 565-0871, Japan \\ $\$$ Frontier Research Base for Global Young Researchers, Graduate School of Engineering, Osaka University, Suita, Osaka 565 - \\ 0871, Japan \\ $\S$ These authors contributed equally to this work. \\ y-nishii@chem.eng.osaka-u.ac.jp \\ miura@chem.eng.osaka-u.ac.jp
}

\section{Contents}

1. General Information $\quad$ S2

2. Experimental Procedure and Identification Data S3

3. X-ray Crystallography $\quad \mathrm{S} 16$

$\begin{array}{ll}\text { 4. DFT Calculation } & \text { S17 }\end{array}$

4. Copy of NMR Spectra $\quad$ S21

5. References $\quad$ S55 


\section{General Information}

All manipulations were performed under $\mathrm{N}_{2}$ using standard Schlenk techniques unless otherwise noted. Toluene, DMF, and 1,4-dioxane were dried and deoxygenated by a Glass Counter Solvent Dispending System (Nikko Hansen \& Co., Ltd.). 1,2-Dichloroethane (DCE), chlorobenzene, trifluoromethylbenzene, and tert-AmOH were distilled from $\mathrm{CaH}_{2}$ and stored with molecular sieves 4A. THF and $\mathrm{MeOH}$ were purchased as dehydrated solvent and used as received. Silica gel column chromatography was performed using Wakosil ${ }^{\circledR}$ C-200 (64-210 $\left.\mu \mathrm{m}\right)$.

Nuclear magnetic resonance spectra were measured at $400 \mathrm{MHz}\left({ }^{1} \mathrm{H} \mathrm{NMR}\right)$, at $100 \mathrm{MHz}\left({ }^{13} \mathrm{C}\right.$ NMR), and at $376 \mathrm{MHz}\left({ }^{19} \mathrm{~F}\right.$ NMR) in $5 \mathrm{~mm}$ NMR tubes. ${ }^{1} \mathrm{H}$ NMR chemical shifts were reported in ppm relative to the resonance of TMS $(\delta 0.00)$ or the residual solvent signals at $\delta 7.26$ for $\mathrm{CDCl}_{3}$, $\delta 2.50$ for DMSO- $d_{6}$, and at $\delta 2.05$ for acetone- $d_{6} .{ }^{13} \mathrm{C}$ NMR chemical shifts were reported in ppm relative to the residual solvent signals at $\delta 77.2$ for $\mathrm{CDCl}_{3}$ and at $\delta 39.5$ for DMSO- $d_{6}$. Melting points were measured with Mettler Toledo MP90. High resolution mass spectra (HRMS) were recorded by APCI-TOF. Preparative gel permeation chromatography (GPC) was conducted with Showa Denko H-2001, H-2002 column eluting with $\mathrm{CHCl}_{3}$. 


\section{Experimental Procedures and Identification Data}

\section{2-1. General Procedure for the Oxidative Annulation of 1 with $\mathbf{2}$ (Scheme 2)}

To an oven-dried screw-top tube were added acid 1 (0.2 mmol), [Cp $\left.{ }^{\mathrm{E}} \mathrm{RhCl}_{2}\right]_{2}(4.3 \mathrm{mg}, 2.5 \mathrm{~mol} \%)$, and $\mathrm{AgSbF}_{6}$ (10.2 mg, $15 \mathrm{~mol} \%$ ). Vinylene carbonate (2) (17.2 mg, $\left.0.2 \mathrm{mmol}\right)$ and DCE (0.5 mL) were added via syringe. The mixture was stirred at $120^{\circ} \mathrm{C}$ for $15 \mathrm{~h}$ under an $\mathrm{N}_{2}$ atmosphere with an oil bath. The resulting solution was diluted with EtOAc and filtered through a pad of silica gel eluting with EtOAc. The filtrate was concentrated in vacuo, and the crude material was purified by silica gel chromatography and, if indicated, GPC to give the corresponding product $\mathbf{3}$.

$1 H$-Isochromen-1-one (3a) ${ }^{1}$<smiles>O=c1occc2ccccc12</smiles>

Purified by silica gel chromatography (hexane/EtOAc $=3 / 1), 21.5 \mathrm{mg}(74 \%$ yield), pale yellow oil; ${ }^{1} \mathrm{H}$ NMR (400 MHz, $\left.\mathrm{CDCl}_{3}\right) \delta 6.51(\mathrm{~d}, J=5.6 \mathrm{~Hz}, 1 \mathrm{H}), 7.28(\mathrm{~d}, J=5.6 \mathrm{~Hz}, 1 \mathrm{H}), 7.44(\mathrm{~d}, J=$ $8.0 \mathrm{~Hz}, 1 \mathrm{H}), 7.51-7.56(\mathrm{~m}, 1 \mathrm{H}), 7.71-7.75(\mathrm{~m}, 1 \mathrm{H}), 8.31(\mathrm{dd}, J=0.5,8.0 \mathrm{~Hz}, 1 \mathrm{H}) ;{ }^{13} \mathrm{C} \mathrm{NMR}(100$ $\left.\mathrm{MHz}, \mathrm{CDCl}_{3}\right) \delta 107.0,121.9,125.5,128.6,129.7,134.8,136.5,144.7,162.2$.

6-Methyl-1H-isochromen-1-one (3b) ${ }^{2}$<smiles>Cc1ccc2c(=O)occc2c1</smiles>

Purified by silica gel chromatography (hexane/EtOAc =10/1), $22.2 \mathrm{mg}(69 \%$ yield), white solid; ${ }^{1} \mathrm{H}$ NMR $\left(400 \mathrm{MHz}\right.$, acetone- $\left.d_{6}\right) \delta 8.09(\mathrm{~d}, J=8.0 \mathrm{~Hz}, 1 \mathrm{H}), 7.42-7.45(\mathrm{~m}, 3 \mathrm{H}), 6.65(\mathrm{~d}, J=5.5 \mathrm{~Hz}$, $1 \mathrm{H}), 2.48$ (s, 3H); ${ }^{13} \mathrm{C} \mathrm{NMR}\left(100 \mathrm{MHz}, \mathrm{CDCl}_{3}\right) \delta 162.4,146.0,144.9,136.7,130.0,129.7,125.7$, $119.5,107.1,22.1$.

6-Methoxy-1H-isochromen-1-one (3c)<smiles>COc1ccc2c(=O)occc2c1</smiles>

Purified by silica gel chromatography (hexane/EtOAc =3/1), $22.5 \mathrm{mg}(64 \%$ yield), white solid, mp 89.8-91.2 ${ }^{\circ} \mathrm{C} ;{ }^{1} \mathrm{H}$ NMR $\left(400 \mathrm{MHz}\right.$, acetone- $\left.d_{6}\right) \delta 8.12(\mathrm{~d}, J=8.8 \mathrm{~Hz}, 1 \mathrm{H}), 7.43(\mathrm{~d}, J=5.6 \mathrm{~Hz}$, $1 \mathrm{H}), 7.15(\mathrm{dd}, J=2.5,8.8 \mathrm{~Hz}, 1 \mathrm{H}), 7.11(\mathrm{~d}, J=2.5 \mathrm{~Hz}, 1 \mathrm{H}), 6.66(\mathrm{~d}, J=5.6 \mathrm{~Hz}, 1 \mathrm{H}), 3.96(\mathrm{~s}, 3 \mathrm{H})$; 
${ }^{13} \mathrm{C}$ NMR $\left(100 \mathrm{MHz}, \mathrm{CDCl}_{3}\right) \delta$ 164.7, 162.0, 145.3, 138.8, 131.9, 116.8, 115.0, 107.7, 107.0, 55.7; HRMS (APCI) $m / z\left([\mathrm{M}+\mathrm{H}]^{+}\right)$calcd for $\mathrm{C}_{10} \mathrm{H}_{9} \mathrm{O}_{3}$ 177.0546, found: 177.0541 .

6-Hydroxy-1H-isochromen-1-one (3d)<smiles>O=c1occc2cc(O)ccc12</smiles>

Purified by silica gel chromatography (hexane/EtOAc $=2 / 1), 19.7 \mathrm{mg}(61 \%$ yield $)$, white solid, mp 213.1-215.1 ${ }^{\circ} \mathrm{C} ;{ }^{1} \mathrm{H}$ NMR (400 MHz, DMSO-d $\left.d_{6}\right) \delta 7.98(\mathrm{~d}, J=8.7 \mathrm{~Hz}, 1 \mathrm{H}), 7.45(\mathrm{~d}, J=5.6$ $\mathrm{Hz}, 1 \mathrm{H}), 6.97(\mathrm{dd}, J=2.2,8.7 \mathrm{~Hz}, 1 \mathrm{H}), 6.87(\mathrm{~d}, J=2.2 \mathrm{~Hz}, 1 \mathrm{H}), 6.64(\mathrm{~d}, J=5.6 \mathrm{~Hz}, 1 \mathrm{H}) ;{ }^{13} \mathrm{C}$ NMR (100 MHz, DMSO- $\left.d_{6}\right) \delta 163.9,161.7,146.2,139.2,132.0,118.1,113.0,110.6,107.1$; HRMS (APCI) $m / z\left([\mathrm{M}+\mathrm{H}]^{+}\right)$calcd for $\mathrm{C}_{9} \mathrm{H}_{7} \mathrm{O}_{3}$ 163.0390, found: 163.0389 .

6-Chloro-1H-isochromen-1-one (3e) ${ }^{2}$<smiles>O=c1occc2cc(Cl)ccc12</smiles>

Purified by silica gel chromatography (hexane/EtOAc $=3 / 1), 23.7 \mathrm{mg}(65 \%$ yield), pale yellow solid; ${ }^{1} \mathrm{H}$ NMR (400 MHz, $\left.\mathrm{CDCl}_{3}\right) \delta 8.23(\mathrm{~d}, J=8.5 \mathrm{~Hz}, 1 \mathrm{H}), 7.48(\mathrm{dd}, J=2.0,8.5 \mathrm{~Hz}, 1 \mathrm{H}), 7.43$ $(\mathrm{d}, J=2.0 \mathrm{~Hz}, 1 \mathrm{H}), 7.31(\mathrm{~d}, J=5.6 \mathrm{~Hz}, 1 \mathrm{H}), 6.44(\mathrm{~d}, J=5.6 \mathrm{~Hz}, 1 \mathrm{H}) ;{ }^{13} \mathrm{C} \mathrm{NMR}\left(100 \mathrm{MHz}, \mathrm{CDCl}_{3}\right)$ $\delta 161.4,146.0,141.5,137.8,131.4,129.1,125.1,120.2,106.1$.

6-Bromo-1H-isochromen-1-one (3f)<smiles>O=c1occc2cc(Br)ccc12</smiles>

Purified by silica gel chromatography (hexane/EtOAc $=5 / 1), 32.9 \mathrm{mg}(73 \%$ yield), white solid, sublimation at $207{ }^{\circ} \mathrm{C} ;{ }^{1} \mathrm{H}$ NMR $\left(400 \mathrm{MHz}, \mathrm{CDCl}_{3}\right) \delta 8.15(\mathrm{~d}, J=8.4 \mathrm{~Hz}, 1 \mathrm{H}), 7.65(\mathrm{dd}, J=1.9$, $8.4 \mathrm{~Hz}, 1 \mathrm{H}), 7.61(\mathrm{~d}, J=1.8 \mathrm{~Hz}, 1 \mathrm{H}), 7.30(\mathrm{~d}, J=5.7 \mathrm{~Hz}, 1 \mathrm{H}), 6.44(\mathrm{~d}, J=5.6 \mathrm{~Hz}, 1 \mathrm{H}) ;{ }^{13} \mathrm{C} \mathrm{NMR}$ $\left(100 \mathrm{MHz}, \mathrm{CDCl}_{3}\right) \delta 161.7,146.1,138.0,132.1,131.5,130.4,128.4,120.7,106.1$; HRMS (APCI) $m / z\left([\mathrm{M}+\mathrm{H}]^{+}\right)$calcd for $\mathrm{C}_{9} \mathrm{H}_{5} \mathrm{BrO}_{2} 224.9546$, found: 224.9545 . 
6-(Trifluoromethyl)-1 $H$-isochromen-1-one (3g)<smiles>O=c1occc2cc(C(F)(F)F)ccc12</smiles>

Purified by silica gel chromatography (hexane/EtOAc $=5 / 1), 23.1 \mathrm{mg}(54 \%$ yield), white solid, mp 149.4-151.4 ${ }^{\circ} \mathrm{C} ;{ }^{1} \mathrm{H}$ NMR $\left(400 \mathrm{MHz}, \mathrm{CDCl}_{3}\right) \delta 8.43(\mathrm{~d}, J=8.3 \mathrm{~Hz}, 1 \mathrm{H}), 7.76(\mathrm{~d}, J=8.3 \mathrm{~Hz}$, $1 \mathrm{H}), 7.72(\mathrm{~s}, 1 \mathrm{H}), 7.37(\mathrm{~d}, J=5.6 \mathrm{~Hz}, 1 \mathrm{H}), 6.58(\mathrm{~d}, J=5.6 \mathrm{~Hz}, 1 \mathrm{H}) ;{ }^{13} \mathrm{C} \mathrm{NMR}\left(100 \mathrm{MHz}, \mathrm{CDCl}_{3}\right)$ $\delta 161.0,146.1,136.8,136.3$ (q, $J=32.8 \mathrm{~Hz}), 130.7,124.9$ (q, $J=3.4 \mathrm{~Hz}), 124.3,123.2$ (q, $J=$ $271.5 \mathrm{~Hz}), 122.8$ (q, $J=3.9 \mathrm{~Hz}$ ); ${ }^{19} \mathrm{~F}$ NMR (376 MHz, $\left.\mathrm{CDCl}_{3}\right) \delta-63.4$; HRMS (APCI) $\mathrm{m} / \mathrm{z}$ $\left([\mathrm{M}+\mathrm{H}]^{+}\right)$calcd for $\mathrm{C}_{10} \mathrm{H}_{6} \mathrm{~F}_{3} \mathrm{O}_{2} 215.0314$, found: 215.0321 .

1-Oxo-1H-isochromene-6-carbaldehyde (3h)<smiles>O=Cc1ccc2c(=O)occc2c1</smiles>

Purified by silica gel chromatography (hexane/EtOAc $=2 / 1), 7.8 \mathrm{mg}(22 \%$ yield), pale yellow solid, mp 178.2-179.2 ${ }^{\circ} \mathrm{C} ;{ }^{1} \mathrm{H}$ NMR $\left(400 \mathrm{MHz}, \mathrm{CDCl}_{3}\right) \delta 10.17(\mathrm{~s}, 1 \mathrm{H}), 8.47(\mathrm{~d}, J=8.1 \mathrm{~Hz}, 1 \mathrm{H})$, $8.00(\mathrm{~d}, J=8.1 \mathrm{~Hz}, 1 \mathrm{H}), 7.95(\mathrm{~d}, J=1.3 \mathrm{~Hz}, 1 \mathrm{H}), 7.36(\mathrm{~d}, J=5.6 \mathrm{~Hz}, 1 \mathrm{H}), 6.62(\mathrm{~d}, J=5.6 \mathrm{~Hz}$, $1 \mathrm{H}) ;{ }^{13} \mathrm{C}$ NMR $\left(100 \mathrm{MHz}, \mathrm{CDCl}_{3}\right) \delta 191.1,161.2,145.8,140.3,137.0,130.8,128.2,127.2,125.8$, 106.7; HRMS (APCI) $m / z\left([\mathrm{M}+\mathrm{H}]^{+}\right)$calcd for $\mathrm{C}_{10} \mathrm{H}_{7} \mathrm{O}_{3}$ 175.0390, found: 175.0384 .

Methyl 1-oxo-1H-isochromene-6-carboxylate (3i)<smiles>CC(=O)c1ccc2c(=O)occc2c1</smiles>

Purified by silica gel chromatography (hexane/EtOAc $=5 / 1)$ and $\mathrm{GPC}\left(\mathrm{CHCl}_{3}\right), 12.8 \mathrm{mg}(31 \%$ yield), white solid, mp 167.0-169.0 ${ }^{\circ} \mathrm{C} ;{ }^{1} \mathrm{H}$ NMR (400 MHz, $\left.\mathrm{CDCl}_{3}\right) \delta 8.37$ (d, J=8.0 Hz, 1H), 8.13-8.15 (m, 2H), $7.33(\mathrm{~d}, J=5.6 \mathrm{~Hz}, 1 \mathrm{H}), 6.58(\mathrm{~d}, J=5.6 \mathrm{~Hz}, 1 \mathrm{H}), 3.99(\mathrm{~s}, 3 \mathrm{H}) ;{ }^{13} \mathrm{C}$ NMR $(100$ $\left.\mathrm{MHz}, \mathrm{CDCl}_{3}\right) \delta 165.9,161.6,145.5,136.6,135.8,130.2,129.0,127.2,124.9,107.0,52.9$; HRMS (APCI) $m / z\left([\mathrm{M}+\mathrm{H}]^{+}\right)$calcd for $\mathrm{C}_{11} \mathrm{H}_{9} \mathrm{O}_{4} 205.0495$, found: 205.0494 . 
7-Bromo-1 $H$-isochromen-1-one (3j)<smiles>O=c1occc2ccc(Br)cc12</smiles>

Purified by silica gel chromatography (hexane/EtOAc $=5 / 1), 24.1 \mathrm{mg}(54 \%$ yield), pale yellow solid, mp 107.8-108.5 ${ }^{\circ} \mathrm{C} ;{ }^{1} \mathrm{H} \mathrm{NMR}\left(400 \mathrm{MHz}, \mathrm{CDCl}_{3}\right) \delta 8.44(\mathrm{~d}, J=2.0 \mathrm{~Hz}, 1 \mathrm{H}), 7.82(\mathrm{dd}, J=$ $2.1,8.4 \mathrm{~Hz}, 1 \mathrm{H}), 7.33(\mathrm{~d}, J=8.4 \mathrm{~Hz}, 1 \mathrm{H}), 7.31(\mathrm{~d}, J=5.6 \mathrm{~Hz}, 1 \mathrm{H}), 6.49(\mathrm{~d}, J=5.6 \mathrm{~Hz}, 1 \mathrm{H}) ;{ }^{13} \mathrm{C}$ NMR (100 MHz, $\left.\mathrm{CDCl}_{3}\right) \delta 161.0,145.2,138.1,135.3,132.4,127.3,123.4,122.2,106.5$; HRMS (APCI) $m / z\left([\mathrm{M}+\mathrm{H}]^{+}\right)$calcd for $\mathrm{C}_{9} \mathrm{H}_{6} \mathrm{BrO}_{2} 224.9546$, found: 224.9539.

8-Methyl-1H-isochromen-1-one (3k)<smiles>Cc1cccc2ccoc(=O)c12</smiles>

Purified by silica gel chromatography (hexane/EtOAc =10/1), $29.3 \mathrm{mg}(91 \%$ yield), white solid, mp 113.2-113.9 ${ }^{\circ} \mathrm{C} ;{ }^{1} \mathrm{H}$ NMR (400 MHz, $\left.\mathrm{CDCl}_{3}\right) \delta 7.55(\mathrm{t}, J=7.5 \mathrm{~Hz}, 1 \mathrm{H}), 7.30(\mathrm{~d}, J=7.4 \mathrm{~Hz}$, $1 \mathrm{H}), 7.21-7.25(\mathrm{~m}, 2 \mathrm{H}), 6.43(\mathrm{~d}, J=5.5 \mathrm{~Hz}, 1 \mathrm{H}), 2.83(\mathrm{~s}, 3 \mathrm{H}) ;{ }^{13} \mathrm{C} \mathrm{NMR}\left(100 \mathrm{MHz}, \mathrm{CDCl}_{3}\right) \delta$ $161.8,144.5,143.8,138.2,134.1,131.6,123.8,120.4,107.6,23.5$; HRMS (APCI) $m / z\left([\mathrm{M}+\mathrm{H}]^{+}\right)$ calcd for $\mathrm{C}_{10} \mathrm{H}_{9} \mathrm{O}_{2}$ 161.0597, found: 161.0593 .

8-Phenyl-1H-isochromen-1-one (3I)<smiles>O=c1occc2cccc(-c3ccccc3)c12</smiles>

Purified by silica gel chromatography (hexane/EtOAc $=10 / 1)$ and GPC $\left(\mathrm{CHCl}_{3}\right), 30.1 \mathrm{mg}(68 \%$ yield), pale yellow solid, mp 127.2-127.9 ${ }^{\circ} \mathrm{C} ;{ }^{1} \mathrm{H} \mathrm{NMR}\left(400 \mathrm{MHz}, \mathrm{CDCl}_{3}\right) \delta 7.70(\mathrm{t}, J=7.7 \mathrm{~Hz}$, 1H), 7.39-7.44 (m, 4H), 7.36 (dd, $J=1.2,9.1 \mathrm{~Hz}, 1 \mathrm{H}), 7.31-7.33(\mathrm{~m}, 2 \mathrm{H}), 7.29$ (d, $J=5.5 \mathrm{~Hz}, 1 \mathrm{H})$, $6.52(\mathrm{~d}, J=5.6 \mathrm{~Hz}, 1 \mathrm{H}) ;{ }^{13} \mathrm{C} \mathrm{NMR}\left(100 \mathrm{MHz}, \mathrm{CDCl}_{3}\right) \delta 160.4,146.2,145.0,141.5,138.1,133.7$, $131.9,128.5,127.8,127.4,125.4,119.1,107.3$; HRMS (APCI) $m / z\left([\mathrm{M}+\mathrm{H}]^{+}\right)$calcd for $\mathrm{C}_{15} \mathrm{H}_{11} \mathrm{O}_{2}$ 223.0754, found: 223.0754 . 
8-Hydroxy-1H-isochromen-1-one (3m) ${ }^{3}$<smiles>O=c1occc2cccc(O)c12</smiles>

Purified by silica gel chromatography (hexane/EtOAc $=5 / 1), 17.8 \mathrm{mg}(55 \%$ yield), white solid; ${ }^{1} \mathrm{H}$ NMR (400 MHz, $\left.\mathrm{CDCl}_{3}\right) \delta 11.02(\mathrm{~s}, 1 \mathrm{H}), 7.62(\mathrm{t}, J=8.1 \mathrm{~Hz}, 1 \mathrm{H}), 7.22(\mathrm{~d}, J=5.6 \mathrm{~Hz}, 1 \mathrm{H})$, $7.01(\mathrm{dd}, J=0.8,8.4 \mathrm{~Hz}, 1 \mathrm{H}), 6.90(\mathrm{dd}, J=0.9,7.6 \mathrm{~Hz}, 1 \mathrm{H}), 6.52(\mathrm{~d}, J=5.6 \mathrm{~Hz}, 1 \mathrm{H}) ;{ }^{13} \mathrm{C}$ NMR $\left(100 \mathrm{MHz}, \mathrm{CDCl}_{3}\right) \delta 166.3,161.8,144.0,137.5,136.8,116.1,115.9,108.5,107.4$.

8-Bromo-1H-isochromen-1-one (3n)<smiles>O=c1occc2cccc(Br)c12</smiles>

Purified by silica gel chromatography (hexane/EtOAc $=3 / 1), 27.5 \mathrm{mg}(61 \%$ yield), beige solid, mp 126.5-127.8 ${ }^{\circ} \mathrm{C} ;{ }^{1} \mathrm{H}$ NMR $\left(400 \mathrm{MHz}, \mathrm{CDCl}_{3}\right) \delta 7.80(\mathrm{dd}, J=1.2,7.8 \mathrm{~Hz}, 1 \mathrm{H}), 7.48(\mathrm{t}, J=7.8$ $\mathrm{Hz}, 1 \mathrm{H}), 7.36(\mathrm{dd}, J=1.2,7.8 \mathrm{~Hz}, 1 \mathrm{H}), 7.28(\mathrm{~d}, J=5.6 \mathrm{~Hz}, 1 \mathrm{H}), 6.47(\mathrm{~d}, J=5.5 \mathrm{~Hz}, 1 \mathrm{H}) ;{ }^{13} \mathrm{C}$ NMR $\left(100 \mathrm{MHz}, \mathrm{CDCl}_{3}\right) \delta 159.1,145.3,139.6,135.4,134.7,125.4,125.0,120.2,107.1$; HRMS (APCI) $m / z\left([\mathrm{M}+\mathrm{H}]^{+}\right)$calcd for $\mathrm{C}_{9} \mathrm{H}_{6} \mathrm{BrO}_{2} 224.9546$, found: 224.9543 .

8-(Trifluoromethyl)-1H-isochromen-1-one (3o)<smiles>O=c1occc2cccc(C(F)(F)F)c12</smiles>

Purified by silica gel chromatography (hexane/EtOAc $=3 / 1), 18.0 \mathrm{mg}(42 \%$ yield), white solid, mp 149.8-150.4 ${ }^{\circ} \mathrm{C} ;{ }^{1} \mathrm{H}$ NMR (400 MHz, $\left.\mathrm{CDCl}_{3}\right) \delta 7.94(\mathrm{~d}, J=7.8 \mathrm{~Hz}, 1 \mathrm{H}), 7.80(\mathrm{dt}, J=0.7,7.9$ $\mathrm{Hz}, 1 \mathrm{H}), 7.64(\mathrm{~d}, J=7.8 \mathrm{~Hz}, 1 \mathrm{H}), 7.36(\mathrm{~d}, J=5.5 \mathrm{~Hz}, 1 \mathrm{H}), 6.53(\mathrm{~d}, J=5.5 \mathrm{~Hz}, 1 \mathrm{H}) ;{ }^{13} \mathrm{C} \mathrm{NMR}$ $\left(100 \mathrm{MHz}, \mathrm{CDCl}_{3}\right) \delta 157.7,145.9,139.5,134.0,131.2(\mathrm{q}, J=32.8 \mathrm{~Hz}), 130.1,127.8(\mathrm{q}, J=7.2$ $\mathrm{Hz}), 122.9$ (q, $J=272.0 \mathrm{~Hz}), 119.0,106.5 ;{ }^{19} \mathrm{~F}$ NMR $(376 \mathrm{MHz}, \mathrm{CDCl} 3) \delta-60.2$; HRMS (APCI) $m / z\left([\mathrm{M}+\mathrm{H}]^{+}\right)$calcd for $\mathrm{C}_{10} \mathrm{H}_{6} \mathrm{~F}_{3} \mathrm{O}_{2} 215.0314$, found: 215.0315 .

6,7-Dimethoxy-1H-isochromen-1-one $(\mathbf{3} \mathbf{p})^{4}$<smiles>COc1cc2ccoc(=O)c2cc1OC</smiles> 
Purified by silica gel chromatography (hexane/EtOAc $=3 / 1), 30.1 \mathrm{mg}(73 \%$ yield), white solid; ${ }^{1} \mathrm{H}$ NMR $\left(400 \mathrm{MHz}, \mathrm{CDCl}_{3}\right) \delta 7.67(\mathrm{~s}, 1 \mathrm{H}), 7.25(\mathrm{~d}, J=5.6 \mathrm{~Hz}, 1 \mathrm{H}), 6.81(\mathrm{~s}, 1 \mathrm{H}), 6.44(\mathrm{~d}, J=5,6$ $\mathrm{Hz}, 1 \mathrm{H}), 4.00(\mathrm{~s}, 3 \mathrm{H}), 3.99$ (s, 3H); ${ }^{13} \mathrm{C} \mathrm{NMR}\left(100 \mathrm{MHz}, \mathrm{CDCl}_{3}\right) \delta 162.4,155.2,150.0,143.9$, 132.2, 115.2, 109.7, 106.8, 106.3, 56.4 (two OMe groups).

9H-[1,3]Dioxolo[4,5-h] isochromen-9-one (3q)<smiles>O=c1occc2c3c(ccc12)OCO3</smiles>

Purified by silica gel chromatography (hexane/EtOAc $=3 / 1), 30.2 \mathrm{mg}(79 \%$ yield), white solid, mp 200.0-201.0 ${ }^{\circ} \mathrm{C} ;{ }^{1} \mathrm{H}$ NMR $\left(400 \mathrm{MHz}, \mathrm{CDCl}_{3}\right) \delta 7.93$ (dd, $\left.J=0.7,8.4 \mathrm{~Hz}, 1 \mathrm{H}\right), 7.23(\mathrm{~d}, J=5,6$ $\mathrm{Hz}, 1 \mathrm{H}), 7.01(\mathrm{~d}, J=8.4 \mathrm{~Hz}, 1 \mathrm{H}), 6.51(\mathrm{dd}, J=0.7,5.6 \mathrm{~Hz}, 1 \mathrm{H}), 6.17(\mathrm{~s}, 2 \mathrm{H}) ;{ }^{13} \mathrm{C} \mathrm{NMR}(100 \mathrm{MHz}$, $\left.\mathrm{CDCl}_{3}\right) \delta 161.4,152.3,145.0,141.7,126.0,119.5,116.0,109.6,102.9,100.4 ;$ HRMS (APCI) $\mathrm{m} / \mathrm{z}$ $\left([\mathrm{M}+\mathrm{H}]^{+}\right)$calcd for $\mathrm{C}_{11} \mathrm{H}_{7} \mathrm{O}_{2} \mathrm{~S} 203.0161$, found: 203.0156 .

5,7-Dimethoxy-1H-isochromen-1-one (3r)<smiles>COc1cc(OC)c2ccoc(=O)c2c1</smiles>

Purified by silica gel chromatography (hexane/EtOAc $=3 / 1), 16.5 \mathrm{mg}(40 \%$ yield), white solid, mp 133.9-135.9 ${ }^{\circ} \mathrm{C} ;{ }^{1} \mathrm{H}$ NMR (400 MHz, $\left.\mathrm{CDCl}_{3}\right) \delta 7.27(\mathrm{~d}, J=2.3 \mathrm{~Hz}, 1 \mathrm{H}), 7.19(\mathrm{~d}, J=5.6 \mathrm{~Hz}$, $1 \mathrm{H}), 6.80(\mathrm{~d}, J=5.6 \mathrm{~Hz}, 1 \mathrm{H}), 6.75(\mathrm{~d}, J=2.3 \mathrm{~Hz}, 1 \mathrm{H}), 3.90(\mathrm{~s}, 6 \mathrm{H}) ;{ }^{13} \mathrm{C} \mathrm{NMR}\left(100 \mathrm{MHz}, \mathrm{CDCl}_{3}\right)$ $\delta 162.5,160.6,155.5,142.1,123.4,121.6,105.2,101.6,101.1,56.1,56.0$; HRMS (APCI) $\mathrm{m} / \mathrm{z}$ $\left([\mathrm{M}+\mathrm{H}]^{+}\right)$calcd for $\mathrm{C}_{11} \mathrm{H}_{11} \mathrm{O}_{4}$ 207.0652, found: 207.0660 .

5,7-Dibromo-1H-isochromen-1-one (3s)<smiles>O=c1occc2c(Br)cc(Br)cc12</smiles>

Purified by silica gel chromatography (hexane/EtOAc $=10 / 1), 19.1 \mathrm{mg}(31 \%$ yield $)$, white solid, mp 158.2-159.2 ${ }^{\circ} \mathrm{C} ;{ }^{1} \mathrm{H}$ NMR $\left(400 \mathrm{MHz}, \mathrm{CDCl}_{3}\right) \delta 8.40-8.41(\mathrm{~m}, 1 \mathrm{H}), 8.08(\mathrm{~d}, J=1.9 \mathrm{~Hz}, 1 \mathrm{H})$, 
$7.37(\mathrm{~d}, J=5.9 \mathrm{~Hz}, 1 \mathrm{H}), 6.82(\mathrm{~d}, J=5.8 \mathrm{~Hz}, 1 \mathrm{H}) ;{ }^{13} \mathrm{C} \mathrm{NMR}\left(100 \mathrm{MHz}, \mathrm{CDCl}_{3}\right) \delta 160.1,146.0$, $140.9,135.1,131.9,124.4,122.1,121.1,105.5$; HRMS (APCI) $m / z\left([\mathrm{M}+\mathrm{H}]^{+}\right)$calcd for $\mathrm{C}_{9} \mathrm{H}_{5} \mathrm{Br}_{2} \mathrm{O}_{2}$ 302.8656, found: 302.8650 .

6-Chloro-8-methyl-1H-isochromen-1-one (3t)<smiles>Cc1cc(Cl)cc2ccoc(=O)c12</smiles>

Purified by silica gel chromatography (hexane/EtOAc $=10 / 1), 32.1 \mathrm{mg}(82 \%$ yield), white solid, mp 127.9-128.5 ${ }^{\circ} \mathrm{C} ;{ }^{1} \mathrm{H}$ NMR (400 MHz, $\left.\mathrm{CDCl}_{3}\right) \delta$ 7.29-7.29 (m, 1H), 7.24-7.25 (m, 2H), 6.37 (d, $J=5.4 \mathrm{~Hz}, 1 \mathrm{H}), 2.80(\mathrm{~s}, 3 \mathrm{H}) ;{ }^{13} \mathrm{C} \mathrm{NMR}\left(100 \mathrm{MHz}, \mathrm{CDCl}_{3}\right) \delta 161.0,146.0,145.7,140.4,139.5$, 131.5, 123.4, 118.8, 106.7, 23.4; HRMS (APCI) $m / z\left([\mathrm{M}+\mathrm{H}]^{+}\right)$calcd for $\mathrm{C}_{10} \mathrm{H}_{8} \mathrm{ClO}_{2}$ 195.0207, found: 195.0205 .

6,8-Dichloro-1H-isochromen-1-one (3u)<smiles>O=c1occc2cc(Cl)cc(Cl)c12</smiles>

Purified by silica gel chromatography (hexane/EtOAc $=5 / 1), 18.1 \mathrm{mg}(42 \%$ yield), white solid, mp 160.1-162.1 ${ }^{\circ} \mathrm{C} ;{ }^{1} \mathrm{H}$ NMR (400 MHz, $\left.\mathrm{CDCl}_{3}\right) \delta 7.54(\mathrm{~d}, J=2.0 \mathrm{~Hz}, 1 \mathrm{H}), 7.32(\mathrm{~d}, J=2.0 \mathrm{~Hz}$, $1 \mathrm{H}), 7.30(\mathrm{~d}, J=5.6 \mathrm{~Hz}, 1 \mathrm{H}), 6.39(\mathrm{~d}, J=5.6 \mathrm{~Hz}, 1 \mathrm{H}) ;{ }^{13} \mathrm{C} \mathrm{NMR}\left(100 \mathrm{MHz}, \mathrm{CDCl}_{3}\right) \delta 158.0$, 146.6, 140.8, 140.3, 138.5, 131.4, 124.3, 117.4, 106.2; HRMS (APCI) $m / z\left([\mathrm{M}+\mathrm{H}]^{+}\right)$calcd for $\mathrm{C}_{9} \mathrm{H}_{5} \mathrm{Cl}_{2} \mathrm{O}_{2} 214.9661$, found: 214.9661 .

$1 H$-Benzo $[g]$ isochromen-1-one $(\mathbf{3 v})^{5}$<smiles>O=c1occc2cc3ccccc3cc12</smiles>

Purified by silica gel chromatography (hexane/EtOAc $=5 / 1), 36.8 \mathrm{mg}(94 \%$ yield), white solid; ${ }^{1} \mathrm{H}$ NMR (400 MHz, $\left.\mathrm{CDCl}_{3}\right) \delta 8.94(\mathrm{~s}, 1 \mathrm{H}), 8.03(\mathrm{~d}, J=8.3 \mathrm{~Hz}, 1 \mathrm{H}), 7.93(\mathrm{~d}, J=8.0 \mathrm{~Hz}, 1 \mathrm{H})$, $7.87(\mathrm{~s}, 1 \mathrm{H}), 7.64-7.68(\mathrm{~m}, 1 \mathrm{H}), 7.55-7.59(\mathrm{~m}, 1 \mathrm{H}), 7.23(\mathrm{~d}, J=5.7 \mathrm{~Hz}, 1 \mathrm{H}), 6.61(\mathrm{~d}, J=5.7 \mathrm{~Hz}$, $1 \mathrm{H}) ;{ }^{13} \mathrm{C} \mathrm{NMR}\left(100 \mathrm{MHz}, \mathrm{CDCl}_{3}\right) \delta 162.5,143.2,136.4,132.5,132.1,131.2,129.7,129.5,127.7$, $126.8,124.0,120.1,107.1$. 
$1 H$-Benzo $[h]$ isochromen-1-one $(\mathbf{3 w})$<smiles>O=c1occc2ccc3ccccc3c12</smiles>

Purified by silica gel chromatography (hexane/EtOAc $=5 / 1), 37.4 \mathrm{mg}(95 \%$ yield), white solid, mp 145.2-145.9 ${ }^{\circ} \mathrm{C} ;{ }^{1} \mathrm{H}$ NMR (400 MHz, $\left.\mathrm{CDCl}_{3}\right) \delta 9.74(\mathrm{~d}, J=8.7 \mathrm{~Hz}, 1 \mathrm{H}), 8.15(\mathrm{~d}, J=8.5 \mathrm{~Hz}$, 1H), 7.92-7.94 (m, 1H), 7.76-7.81 (m, 1H), 7.63-7.67(m, 1H), $7.51(\mathrm{~d}, J=5.4 \mathrm{~Hz}, 1 \mathrm{H}), 7.47(\mathrm{~d}, J$ $=8.5 \mathrm{~Hz}, 1 \mathrm{H}), 6.66(\mathrm{~d}, J=5.3 \mathrm{~Hz}, 1 \mathrm{H}) ;{ }^{13} \mathrm{C} \mathrm{NMR}\left(100 \mathrm{MHz}, \mathrm{CDCl}_{3}\right) \delta 161.7,146.3,139.2,136.6$, 133.2, 131.7, 129.6, 128.8, 127.1, 126.8, 123.5, 115.6, 107.9; HRMS (APCI) $m / z\left([\mathrm{M}+\mathrm{H}]^{+}\right)$calcd for $\mathrm{C}_{13} \mathrm{H}_{9} \mathrm{O}_{2}, 197.0597$, found: 197.0597.

$1 H$-Benzo[4,5]thieno[2,3-c]pyran-1-one (3x)<smiles>O=c1occc2c1sc1ccccc12</smiles>

Purified by silica gel chromatography (hexane/EtOAc $=3 / 1), 19.2 \mathrm{mg}(47 \%$ yield), pale yellow solid, mp 159.9-161.9 ${ }^{\circ} \mathrm{C} ;{ }^{1} \mathrm{H}$ NMR (400 MHz, $\left.\mathrm{CDCl}_{3}\right) \delta$ 8.03-8.06 (m, 1H), 7.97-8.00 (m, 1H), 7.60-7.65 (m, 2H), 7.53-7.57 (m, 1H), 7.01 (d, $J=5.4 \mathrm{~Hz}, 1 \mathrm{H}) ;{ }^{13} \mathrm{C}$ NMR (100 MHz, DMSO- $\left.d_{6}\right)$ $\delta 158.5,149.2,143.0,142.2,134.0,129.6,125.8,124.8,123.9,123.0,102.4$; HRMS (APCI) $m / z$ $\left([\mathrm{M}+\mathrm{H}]^{+}\right)$calcd for $\mathrm{C}_{11} \mathrm{H}_{7} \mathrm{O}_{2} \mathrm{~S} 203.0161$, found: 203.0169 .

$1 H$-Benzo[4,5]thieno[3,2-c]pyran-1-one (3y)<smiles>O=c1occc2sc3ccccc3c12</smiles>

Purified by silica gel chromatography (hexane/EtOAc $=5 / 1), 19.5 \mathrm{mg}(48 \%$ yield), yellow solid, mp 164.8-166.8 ${ }^{\circ} \mathrm{C} ;{ }^{1} \mathrm{H}$ NMR $\left(400 \mathrm{MHz}, \mathrm{CDCl}_{3}\right) \delta 8.65(\mathrm{~d}, J=8.0 \mathrm{~Hz}, 1 \mathrm{H}), 7.89(\mathrm{~d}, J=8.0 \mathrm{~Hz}$, $1 \mathrm{H}), 7.55-7.60(\mathrm{~m}, 2 \mathrm{H}), 7.48-7.52(\mathrm{~m}, 1 \mathrm{H}), 6.84(\mathrm{~d}, J=5.5 \mathrm{~Hz}, 1 \mathrm{H}) ;{ }^{13} \mathrm{C} \mathrm{NMR}\left(100 \mathrm{MHz}, \mathrm{CDCl}_{3}\right)$ $\delta 157.9,151.4,147.9,137.8,135.5,126.6,126.4,124.9,122.3,118.8,103.6 ; \mathrm{HRMS}($ APCI) m/z $([\mathrm{M}+\mathrm{H}]+)$ calcd for $\mathrm{C}_{11} \mathrm{H}_{7} \mathrm{O}_{2} \mathrm{~S} 203.0161$, found: 203.0156 . 


\section{2-2. Oxidative Annulation of $\mathbf{1} \mathbf{w}$ with $\mathbf{2}$ in $1.0 \mathrm{mmol}$ scale (Scheme 2)}

To an oven-dried screw-top tube were added acid $\mathbf{1 w}(172.2 \mathrm{mg}, 1.0 \mathrm{mmol}),\left[\mathrm{Cp}^{\mathrm{E}} \mathrm{RhCl}_{2}\right]_{2}(21.3$ $\mathrm{mg}, 2.5 \mathrm{~mol} \%$ ), and $\mathrm{AgSbF}_{6}$ (51.5 mg, $15 \mathrm{~mol} \%$ ). Vinylene carbonate (2) (86.0 mg, $1.0 \mathrm{mmol}$ ) and DCE $(2.5 \mathrm{~mL})$ were added via syringe. The mixture was stirred at $120^{\circ} \mathrm{C}$ for $15 \mathrm{~h}$ under an $\mathrm{N}_{2}$ atmosphere with an oil bath. The resulting solution was diluted with EtOAc and filtered through a pad of silica gel eluting with EtOAc. The filtrate was concentrated in vacuo, and the crude material was purified by silica gel chromatography (hexane/EtOAc $=5 / 1)$ to give $\mathbf{3 w}$ in $79 \%$ yield $(155.4$ $\mathrm{mg}$ ) as white solid.

\section{2-3. Hydrogenation of $\mathbf{3 w}$ (Scheme 3 )}<smiles>O=c1occc2ccc3ccccc3c12</smiles>

$3 w$

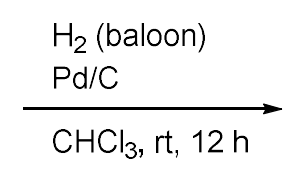<smiles>O=C1OCCc2ccc3ccccc3c21</smiles>

4, 96\% yield

To a round-bottom flask equipped with an $\mathrm{H}_{2}$ balloon were added $\mathbf{3 w}$ (19.6 mg, $\left.0.1 \mathrm{mmol}\right), \mathrm{Pd} / \mathrm{C}$ $(10 \% \mathrm{Pd}, 4.2 \mathrm{mg})$, and $\mathrm{CHCl}_{3}(2.0 \mathrm{~mL})$. The resulting suspension was stirred at room temperature for $12 \mathrm{~h}$ under $\mathrm{H}_{2}$. The mixture was filtered through a pad of Celite eluting with $\mathrm{CHCl}_{3}$, and the filtrate was concentrated in vacuo. The crude material was purified by silica gel chromatography (hexane/EtOAc $=5 / 1)$ to give 4 in $96 \%$ yield $(19.0 \mathrm{mg}$ ) as white solid.

\section{3,4-dihydro-1 $H$-benzo $[h]$ isochromen-1-one (4) ${ }^{6}$}

${ }^{1} \mathrm{H}$ NMR $\left(400 \mathrm{MHz}, \mathrm{CDCl}_{3}\right) \delta 9.11(\mathrm{~d}, J=8.8 \mathrm{~Hz}, 1 \mathrm{H}), 8.02(\mathrm{~d}, J=8.3 \mathrm{~Hz}, 1 \mathrm{H}), 7.87(\mathrm{~d}, J=8.2$ $\mathrm{Hz}, 1 \mathrm{H}), 7.65-7.69(\mathrm{~m}, 1 \mathrm{H}), 7.53-7.57(\mathrm{~m}, 1 \mathrm{H}), 7.35(\mathrm{~d}, J=8.4 \mathrm{~Hz}, 1 \mathrm{H}), 4.55$ (t, $J=5.9 \mathrm{~Hz}, 2 \mathrm{H})$, $3.20(\mathrm{t}, J=5.9 \mathrm{~Hz}, 2 \mathrm{H}) ;{ }^{13} \mathrm{C} \mathrm{NMR}\left(100 \mathrm{MHz}, \mathrm{CDCl}_{3}\right) \delta 164.4,141.5,134.7,133.3,132.1,128.9$, $128.7,126.4$ (2C), 124.9, 120.4, 66.3, 29.7.

\section{2-4. Epoxidation of 3w (Scheme 3)}

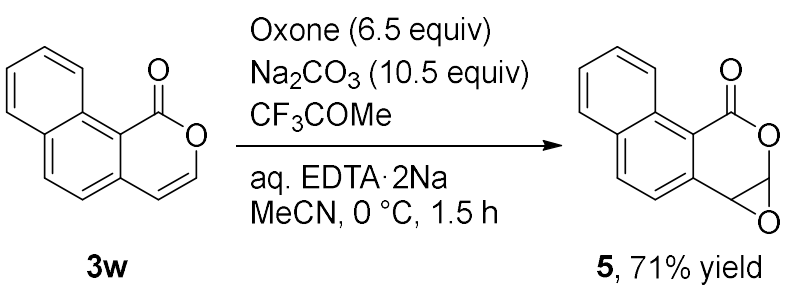

To a solution of $\mathbf{3 w}(39.2 \mathrm{mg}, 0.2 \mathrm{mmol})$ in $\mathrm{MeCN}(1.5 \mathrm{~mL})$ was added an aqueous EDTA·2Na 
$\left(1.0 \mathrm{~mL}, 4 \times 10^{-4} \mathrm{~mol} / \mathrm{L}\right)$. The resulting solution was cooled to $0{ }^{\circ} \mathrm{C}$, followed by the addition of trifluoroacetone $(0.2 \mathrm{~mL})$ via a precooled syringe. $\mathrm{Na}_{2} \mathrm{CO}_{3}(176 \mathrm{mg}, 2.1 \mathrm{mmol})$ and Oxone (198 $\mathrm{mg}, 1.3 \mathrm{mmol}$ ) were added portionwise to the solution over a period of $1 \mathrm{~h}$. The progress of the reaction was monitored on TLC. After stirring $1.5 \mathrm{~h}$ at the temperature, the resulting mixture was poured into water $(20 \mathrm{~mL})$, extracted with EtOAc, dried over $\mathrm{Na}_{2} \mathrm{SO}_{4}$, and concentrated in vacuo. The residue was purified by silica gel chromatography (hexane/EtOAc $=3 / 1$ ) to give 5 in $71 \%$ yield $(30.0 \mathrm{mg})$ as white solid.

1a,9a-Dihydro- $8 H$-benzo[ $h]$ oxireno[2,3-c]isochromen-8-one (5)

m.p. $152-155^{\circ} \mathrm{C} ;{ }^{1} \mathrm{H}$ NMR $\left(400 \mathrm{MHz}, \mathrm{CDCl}_{3}\right) \delta 9.54(\mathrm{~d}, J=8.8 \mathrm{~Hz}, 1 \mathrm{H}), 8.16(\mathrm{~d}, J=8.4 \mathrm{~Hz}, 1 \mathrm{H})$, $7.92(\mathrm{~d}, J=8.4 \mathrm{~Hz}, 1 \mathrm{H}), 7.57-7.76(\mathrm{~m}, 3 \mathrm{H}), 5.83(\mathrm{~d}, J=2.4 \mathrm{~Hz}, 1 \mathrm{H}), 4.21(\mathrm{~d}, J=2.4 \mathrm{~Hz}, 1 \mathrm{H}) ;{ }^{13} \mathrm{C}$ NMR $\left(100 \mathrm{MHz}, \mathrm{CDCl}_{3}\right) \delta 160.4,138.4,135.9,134.6,132.4,129.8,129.0,127.5,126.9,125.9$, 118.1, 50.94, 50.93; HRMS (APCI) $m / z\left([\mathrm{M}+\mathrm{H}]^{+}\right)$calcd for $\mathrm{C}_{10} \mathrm{H}_{9} \mathrm{BrNO}_{2}$ 253.9811, found 253.9811 .

\section{2-5. Bromination of $3 w$ (Scheme 3)}

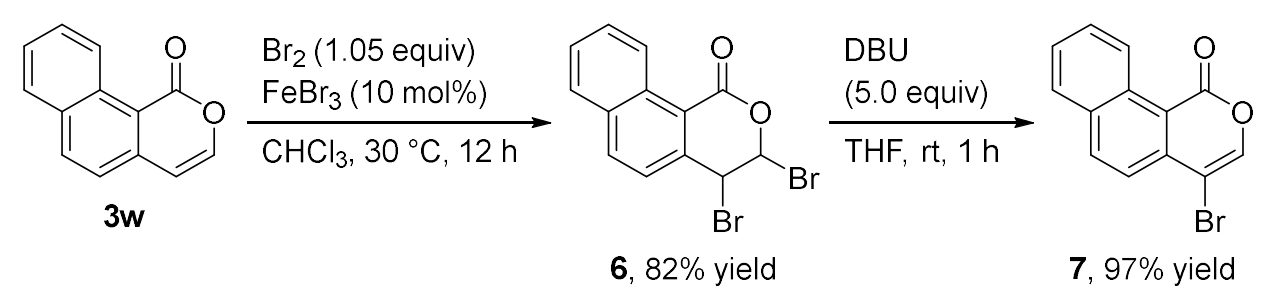

To a round-bottom flask equipped with an $\mathrm{N}_{2}$ balloon were added $\mathbf{3 w}$ (19.6 $\left.\mathrm{mg}, 0.1 \mathrm{mmol}\right)$ and $\mathrm{FeBr}_{3}(3.0 \mathrm{mg}, 0.01 \mathrm{mmol}) . \mathrm{CHCl}_{3}(1.0 \mathrm{~mL})$ was added via syringe. Bromine $(16.8 \mathrm{mg}, 0.105$ mmol) solution (in $0.5 \mathrm{~mL} \mathrm{CHCl}_{3}$ ) was added dropwise. After stirring at $30^{\circ} \mathrm{C}$ for $12 \mathrm{~h}$ with an oil bath, $\mathrm{Na}_{2} \mathrm{~S}_{2} \mathrm{O}_{3}$ aq was added. The resulting mixture was extracted with $\mathrm{CHCl}_{3}$ three times, and the combined organic layers was dried over $\mathrm{Na}_{2} \mathrm{SO}_{4}$, concentrated in vacuo, and purified by silica gel chromatography (hexane/EtOAc $=10 / 1)$ to give 6 in $82 \%$ yield $(29.3 \mathrm{mg}$ ) as white solid.

trans-3,4-Dibromo-3,4-dihydro-1 $H$-benzo[ $h]$ isochromen-1-one (6)

mp 154.8-156.8 ${ }^{\circ} \mathrm{C} ;{ }^{1} \mathrm{H}$ NMR $\left(400 \mathrm{MHz}, \mathrm{CDCl}_{3}\right) \delta 9.26(\mathrm{~d}, J=8.7 \mathrm{~Hz}, 1 \mathrm{H}), 8.20(\mathrm{~d}, J=8.4 \mathrm{~Hz}$, $1 \mathrm{H}), 7.93(\mathrm{~d}, J=8.1 \mathrm{~Hz}, 1 \mathrm{H}), 7.76(\mathrm{dt}, J=1.2,7.8 \mathrm{~Hz}, 1 \mathrm{H}), 7.66(\mathrm{t}, J=7.5 \mathrm{~Hz}, 1 \mathrm{H}), 7.49$ (d, $J=$ $8.4 \mathrm{~Hz}, 1 \mathrm{H}), 6.98(\mathrm{~d}, J=1.1 \mathrm{~Hz}, 1 \mathrm{H}), 5.62(\mathrm{~d}, J=1.2 \mathrm{~Hz}, 1 \mathrm{H}) ;{ }^{13} \mathrm{C}$ NMR $\left(100 \mathrm{MHz}, \mathrm{CDCl}_{3}\right) \delta$ 
$158.9,138.8,136.9,134.5,131.7,130.2,129.1,128.1,126.7,124.6,118.0,79.1,44.9$; HRMS (APCI) $m / z\left([\mathrm{M}+\mathrm{H}]^{+}\right)$calcd for $\mathrm{C}_{13} \mathrm{H}_{9} \mathrm{Br}_{2} \mathrm{O}_{4} 354.8964$, found: 354,8977 .

To a screw-top vial were added $6(8.9 \mathrm{mg}, 0.025 \mathrm{mmol})$ and THF $(1.0 \mathrm{~mL})$. The solution was cooled with ice-water bath, and DBU $(19.0 \mathrm{mg}, 0.125 \mathrm{mmol})$ was added. After stirring at room temperature for $1 \mathrm{~h}, \mathrm{NH}_{4} \mathrm{Claq}$ was added. The resulting mixture was extracted with EtOAc three times, and the combined organic layers was dried over $\mathrm{Na}_{2} \mathrm{SO}_{4}$, concentrated in vacuo, and purified by silica gel chromatography (hexane/EtOAc $=20 / 1)$ to give 7 in $97 \%$ yield $(6.7 \mathrm{mg}$ ) as white solid. Single crystals suitable for the diffraction analysis were obtained by slow evaporation of the $\mathrm{CHCl}_{3}$ solution.

4-Bromo-1 $H$-benzo[ $h]$ isochromen-1-one (7)

mp 159.9-161.2 ${ }^{\circ} \mathrm{C} ;{ }^{1} \mathrm{H}$ NMR (400 MHz, $\left.\mathrm{CDCl}_{3}\right) \delta 9.77(\mathrm{~d}, J=8.8 \mathrm{~Hz}, 1 \mathrm{H}), 8.27(\mathrm{~d}, J=8.8 \mathrm{~Hz}$, 1H), 7.98 (d, $J=8.0 \mathrm{~Hz}, 1 \mathrm{H}), 7.91$ (d, $J=8.4 \mathrm{~Hz}, 1 \mathrm{H}), 7.82$ (t, $J=7.8 \mathrm{~Hz}, 1 \mathrm{H}), 7.74(\mathrm{~s}, 1 \mathrm{H}), 7.70$ (t, $J=7.5 \mathrm{~Hz}, 1 \mathrm{H}) ;{ }^{13} \mathrm{C} \mathrm{NMR}\left(100 \mathrm{MHz}, \mathrm{CDCl}_{3}\right) \delta 160.3,144.7,137.3$ (2C overlapped), 133.6, $131.5,130.2,129.0,127.9,126.9,123.0,115.9,104.0$; HRMS (APCI) $m / z\left([\mathrm{M}+\mathrm{H}]^{+}\right)$calcd for $\mathrm{C}_{13} \mathrm{H}_{8} \mathrm{BrO}_{4}$ 274.9702, found: 274,9714.

\section{$\underline{2-6 .}$ Ring-opening reaction of $\mathbf{3}$ w using Grignard reagent (Scheme 3)}

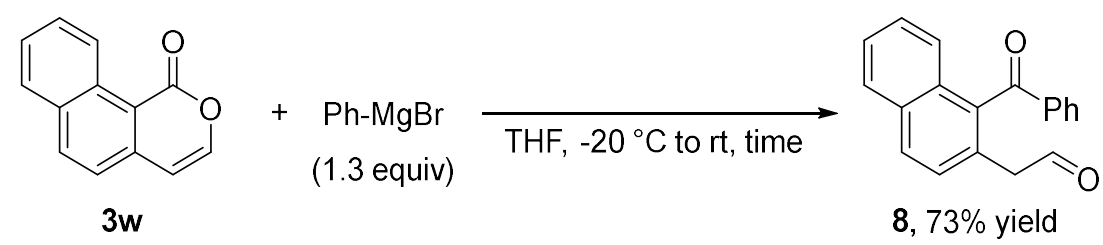

To a solution of $\mathbf{3 w}(39.2 \mathrm{mg}, 0.2 \mathrm{mmol})$ in THF $(2.0 \mathrm{~mL})$ was added $\mathrm{PhMgBr}(0.26 \mathrm{mmol}, 1.0$ $\mathrm{mol} / \mathrm{L}$ solution in THF) at $-20^{\circ} \mathrm{C}$. The mixture was allowed to warm to room temperature and sirried at this temperature for additional $1.5 \mathrm{~h}$. The reaction was quenched with $\mathrm{NH}_{4} \mathrm{Claq}$, and extracted with EtOAc. The combined organic layers was dried over $\mathrm{Na}_{2} \mathrm{SO}_{4}$, concentrated in vacuo, and purified by silica gel chromatography (hexane/EtOAc $=3 / 1)$ to give 8 in $73 \%$ yield $(40 \mathrm{mg}$ ) as yellow oil.

2-(1-Benzoylnaphthalen-2-yl)acetaldehyde (8)

${ }^{1} \mathrm{H}$ NMR $\left(400 \mathrm{MHz}, \mathrm{CDCl}_{3}\right) \delta{ }^{1} \mathrm{H} \mathrm{NMR}\left(400 \mathrm{MHz}, \mathrm{CDCl}_{3}\right) \delta 9.66(\mathrm{t}, J=2.0 \mathrm{~Hz}, 1 \mathrm{H}), 7.96(\mathrm{~d}, J$ $=8.4 \mathrm{~Hz}, 1 \mathrm{H}), 7.90(\mathrm{~d}, J=8.0 \mathrm{~Hz}, 1 \mathrm{H}), 7.80(\mathrm{~d}, J=7.2 \mathrm{~Hz}, 2 \mathrm{H}), 7.34-7.62(\mathrm{~m}, 7 \mathrm{H}), 3.71(\mathrm{~d}, J=$ 
$2.0 \mathrm{~Hz}, 2 \mathrm{H}) ;{ }^{13} \mathrm{C} \mathrm{NMR}\left(100 \mathrm{MHz}, \mathrm{CDCl}_{3}\right) \delta 199.4,198.4,137.6,137.5,134.3,132.4,130.8,130.0$, 129.9, 129.0, 128.3, 128.0, 127.13, 127.09, 126.5, 125.5, 48.3; HRMS (APCI) $m / z\left([\mathrm{M}+\mathrm{H}]^{+}\right)$calcd for $\mathrm{C}_{19} \mathrm{H}_{15} \mathrm{O}_{2} 275.1067$, found 275.1068 .

\section{2-7. Synthesis of 2-Bromonorketoyobyrine (10) (Scheme 4)}

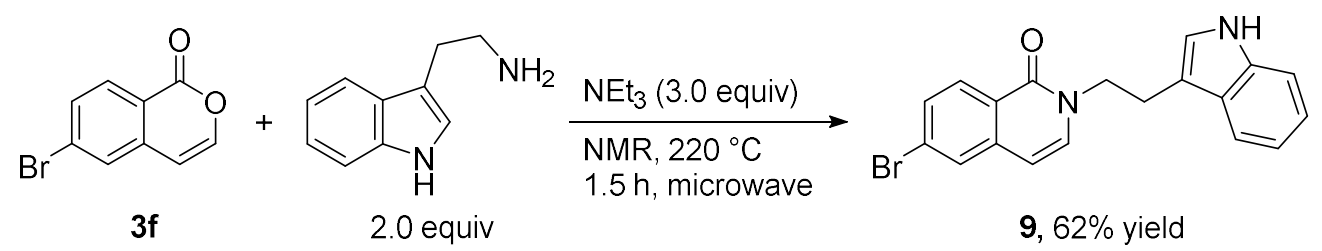

A solution of $\mathbf{3 f}$ ( $22 \mathrm{mg}, 0.1 \mathrm{mmol}$ ), tryptamine (32 $\mathrm{mg}, 0.2 \mathrm{mmol}), \mathrm{Et}_{3} \mathrm{~N}$ (42 mL, $0.3 \mathrm{mmol}$ ) and NMP $(0.5 \mathrm{~mL})$ in a sealed tube was heated at $220{ }^{\circ} \mathrm{C}$ under microwave irradiation (adopting Biotage ${ }^{\circledR}$ Initiator + ) for $1.5 \mathrm{~h}$. The resulting mixture was then poured into water and extracted with ethyl acetate. The combined organic layers was dried over $\mathrm{Na}_{2} \mathrm{SO}_{4}$, concentrated in vacuo, and purified by silica gel chromatography (hexane/EtOAc $=3 / 1)$ to give 9 in $62 \%$ yield $(23 \mathrm{mg})$ as yellow semisolid. This compound could be prepared under conventional heating (at $120{ }^{\circ} \mathrm{C}$ for 3 $\mathrm{h}$ in $\mathrm{MeOH}$ solvent with an oil bath) in $33 \%$ yield.

2-(2-(1H-indol-3-yl)ethyl)-6-bromoisoquinolin-1(2H)-one (9)

${ }^{1} \mathrm{H}$ NMR $\left(400 \mathrm{MHz}, \mathrm{CDCl}_{3}\right) \delta 8.32(\mathrm{~d}, J=8.8 \mathrm{~Hz}, 1 \mathrm{H}), 8.14(\mathrm{~s}, 1 \mathrm{H}), 7.67(\mathrm{~d}, J=8.0 \mathrm{~Hz}, 1 \mathrm{H}), 7.62$ $(\mathrm{d}, J=2.0 \mathrm{~Hz}, 1 \mathrm{H}), 7.56(\mathrm{dd}, J=1.8,8.8 \mathrm{~Hz}, 1 \mathrm{H}), 7.37(\mathrm{~d}, J=8.0 \mathrm{~Hz}, 1 \mathrm{H}), 7.09-7.24(\mathrm{~m}, 2 \mathrm{H})$, $6.91(\mathrm{~d}, J=2.4 \mathrm{~Hz}, 1 \mathrm{H}), 6.19(\mathrm{~d}, J=7.6 \mathrm{~Hz}, 1 \mathrm{H}), 4.27(\mathrm{t}, J=7.2 \mathrm{~Hz}, 2 \mathrm{H}), 3.24(\mathrm{t}, J=7.2 \mathrm{~Hz}, 2 \mathrm{H})$; ${ }^{13} \mathrm{C} \mathrm{NMR}\left(100 \mathrm{MHz}, \mathrm{CDCl}_{3}\right) \delta 161.7,138.6,136.4,133.6,129.9,129.7,128.3,127.2,127.1,124.9$, 122.6, 122.2 119.6, 118.6, 112.0, 111.3, 104.3, 50.5, 24.9; HRMS (APCI) $m / z\left([\mathrm{M}+\mathrm{H}]^{+}\right)$calcd for $\mathrm{C}_{19} \mathrm{H}_{16} \mathrm{BrN}_{2} \mathrm{O}^{+} 367.0441$, found: 367.0446 . 


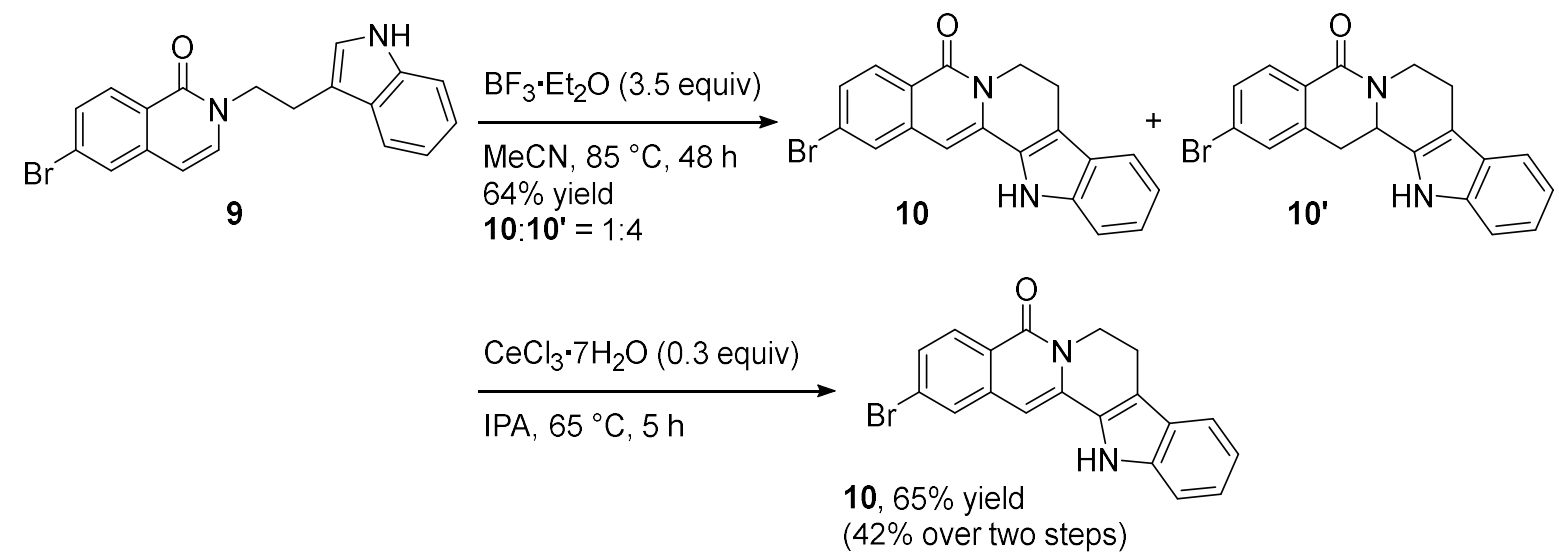

A screw-top tube was charged with the solution of 9 (37 mg, $0.1 \mathrm{mmol})$ in $\mathrm{MeCN}(2.0 \mathrm{~mL})$, and $\mathrm{BF}_{3} \cdot \mathrm{Et}_{2} \mathrm{O}(43 \mu \mathrm{L}, 0.35 \mathrm{mmol})$ was added via stirring. The tube was sealed with a PTFE screw stopper, and the mixture was hated at $85^{\circ} \mathrm{C}$ for $48 \mathrm{~h}$ with an oil bath. After cooling to room temperature, volatiles are removed under reduced pressure, and the residue was purified by silica gel chromatography $\left(\mathrm{CHCl}_{3}\right) .{ }^{1} \mathrm{H}$ NMR spectrum indicated that it constituted of the target product 10 and its saturated analogue 10' in a ratio of 1:4. The total yield was 64\% (23 mg).

The mixture $(37 \mathrm{mg}, \mathbf{1 0}: 10 '=1: 4,0.1 \mathrm{mmol})$ was dissolved isopropanol $(4.0 \mathrm{~mL})$ while heating at $65{ }^{\circ} \mathrm{C}$ with an oil bath. To this solution was added $\mathrm{CeCl}_{3} \cdot 7 \mathrm{H}_{2} \mathrm{O}(12 \mathrm{mg}, 0.03 \mathrm{mmol})$, and the mixture was stirred at this temperature for $5 \mathrm{~h}$. The resulting mixture was neutralized with $\mathrm{NaHCO}_{3} \mathrm{aq}$ and extracted with EtOAc. The combined organic layers was dried over $\mathrm{Na}_{2} \mathrm{SO}_{4}$, concentrated in vacuo, and purified by silica gel chromatography $\left(\mathrm{CHCl}_{3}\right)$ to give $\mathbf{1 0}$ in $65 \%$ yield as yellow solid (24 mg, 65\%).

2-Bromonorketoyobyrine (10)

mp $280{ }^{\circ} \mathrm{C}$ (decomp.); ${ }^{1} \mathrm{H}$ NMR (400 MHz, DMSO- $\left.d_{6}\right) \delta 11.68(\mathrm{~s}, 1 \mathrm{H}), 8.06$ (d, $\left.J=8.4 \mathrm{~Hz}, 1 \mathrm{H}\right)$, $7.77(\mathrm{~d}, J=2.0 \mathrm{~Hz}, 1 \mathrm{H}), 7.49-7.56(\mathrm{~m}, 2 \mathrm{H}), 7.38(\mathrm{~d}, J=8.0 \mathrm{~Hz}, 1 \mathrm{H}), 7.12-7.20(\mathrm{~m}, 1 \mathrm{H}), 6.96-7.04$ $(\mathrm{m}, 1 \mathrm{H}), 6.91(\mathrm{~s}, 1 \mathrm{H}), 4.31(\mathrm{t}, J=6.4 \mathrm{~Hz}, 2 \mathrm{H}), 3.03(\mathrm{t}, J=6.8 \mathrm{~Hz}, 2 \mathrm{H}) ;{ }^{13} \mathrm{C} \mathrm{NMR}(100 \mathrm{MHz}$, DMSO- $\left.d_{6}\right) \delta 161.3,138.6,138.4,134.2,130.3,129.4,128.31,128.27,127.2,125.9,124.3,123.7$, 120.1, 119.8, 113.7, 112.2, 98.3, 40.9, 19.7; HRMS (APCI) $m / z\left([\mathrm{M}+\mathrm{H}]^{+}\right)$calcd for $\mathrm{C}_{19} \mathrm{H}_{14} \mathrm{BrN}_{2} \mathrm{O}$ 365.0284 found 365.0294 . 


\section{X-ray Crystallography}

The structures were refined on $F^{2}$ by full-matrix least-squares method, using SHELXL-2016/6. ${ }^{7}$ Hydrogen atoms were included in the refinement on calculated positions riding on their carrier atoms. ORTEP- $3^{8}$ programs were used to draw the molecules. Single crystals of 7 suitable for the diffraction analysis were obtained by slow evaporation of the $\mathrm{CHCl}_{3}$ solution.

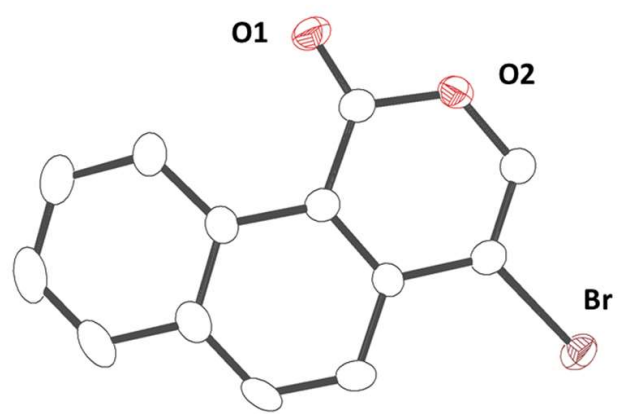

Figure S1. ORTEP drawing for 7 with 50\% thermal ellipsoid. Hydrogen atoms are omitted for clarity.

Table S1. Crystal data for 7

CCDC No.

Crystal system

Space group

Unit cell parameter $[\AA, \operatorname{deg}]$

Z

$\mathrm{R}$ factor $(I>2.0 \sigma(I))$

$\mathrm{R}$ factor (all data)

Goodness of fit

Rint
2008750

monoclinic

$P 2{ }_{1} / c$ (No. 14)

$\mathrm{a}=7.0722(3)$

$\mathrm{b}=8.7072(3), \beta=89.358(3)$

$\mathrm{c}=16.3646(5)$

4

$R 1=0.0452, w R 2=0.1216$

$R 1=0.0481, w R 2=0.1332$

1.064

0.0523 


\section{DFT Calculation}

All calculations were carried out using the Gaussian 16 program, ${ }^{9}$ and geometries of all optimized structures are summarized in an XYZ file. The long-range and dispersion corrected $\omega \mathrm{B} 97 \mathrm{X}-\mathrm{D}$ functional was employed for this study. ${ }^{10}$ Preliminary calculations were carried out with Def2SVP (for H, C, O) and Lanl2DZ (for Rh) basis sets without considering the solvation. For the further refinement, 6-31+G(d,p) basis set (for H, C, O) was used, and the solvent effect of 1,2dichloroethane was taken account by the integral equation formalism PCM (IEF-PCM). Enthalpies and free energies at the optimized structures were calculated with $6-311+G(d, p)$ (for $H, C, O$ atoms) and SDD (for Rh with an additional f polarization function $\left.\zeta_{\mathrm{f}(\mathrm{Rh})}=1.350\right)^{11}$ basis sets. The optimized molecular structures were verified by vibrational analysis; equilibrium structures did not have imaginary frequencies and transition state structures had only one imaginary frequency corresponding to the reaction coordinate. Additionally, the intrinsic reaction coordinate (IRC) calculations were carried out to check whether the transition state leading to the reactant and the product.

To simplify the calculation, $-\mathrm{CO}_{2} \mathrm{Et}$ groups on the $\mathrm{Cp}^{\mathrm{E}}$ ligand were replaced with $-\mathrm{CO}_{2} \mathrm{Me}$, and AcOH was employed as a supporting ligand instead of $\mathrm{PhCO}_{2} \mathrm{H}$ and $\mathrm{H}_{2} \mathrm{CO}_{3}$. The orientation of the substituents on the $\mathrm{Cp}^{\mathrm{E}}$ ligand exerted minimal impact on the calculation because ca. $0.5 \mathrm{kcal} / \mathrm{mol}$ total energy change observed by changing the positions. 


\section{Preliminary study 1}

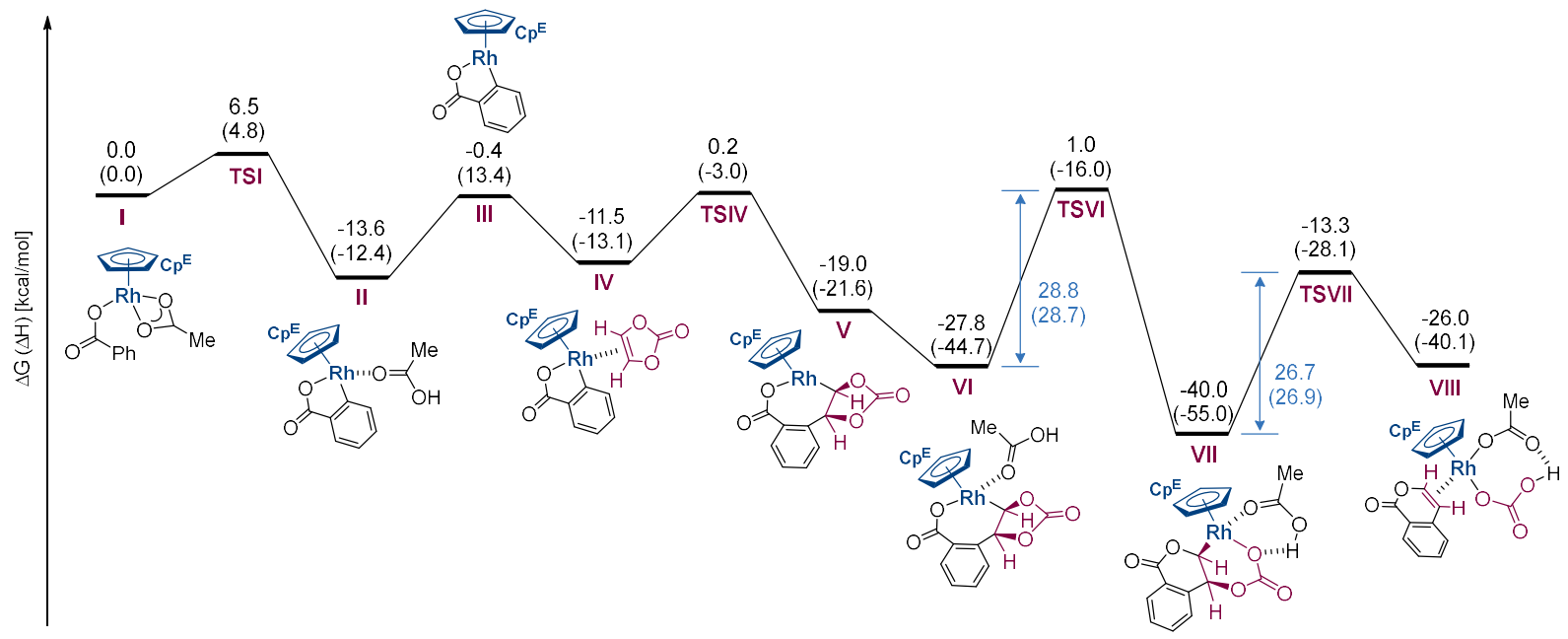

Figure S2. Gibbs energy profile of the reaction. Values in parentheses are relative enthalpies. The calculations were conducted at the $\omega$ B97X-D/Def2SVP\&Lanl2DZ level.

\section{Preliminary study 2}

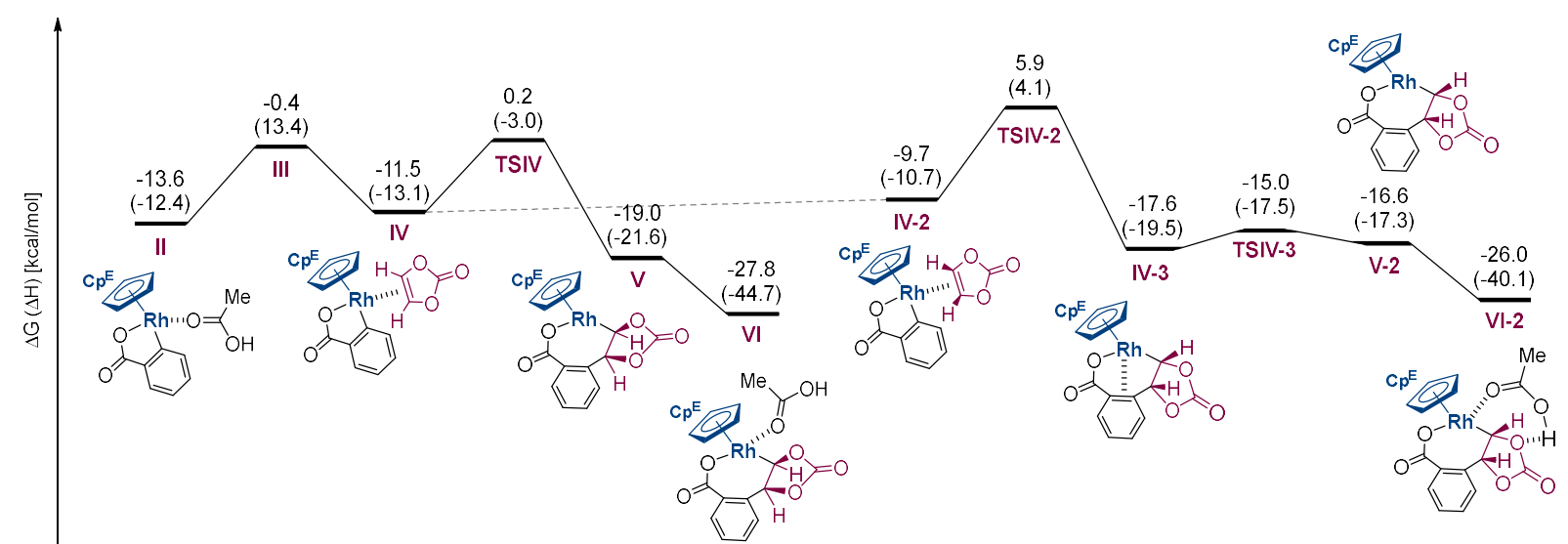

Figure S3. Gibbs energy profile of the reaction. Values in parentheses are relative enthalpies. The calculations were conducted at the $\omega$ B97X-D/Def2SVP\&Lanl2DZ level. 
Table S2. Summary of the calculation conducted at the $\omega$ B97X-D/Def2SVP\&Lanl2DZ level.

\begin{tabular}{|c|c|c|c|}
\hline & $\begin{array}{l}\text { EE + Thermal Enthalpy } \\
\text { Correction [Hartree] }\end{array}$ & $\begin{array}{l}\text { EE + Thermal Free Energy } \\
\text { Correction [Hartree] }\end{array}$ & $\begin{array}{l}\text { Number of Imaginary } \\
\text { Frequency }\end{array}$ \\
\hline $\mathrm{AcOH}$ & -228.77777 & -228.81046 & 0 \\
\hline carbonate & -340.75015 & -340.78284 & 0 \\
\hline 1 & -1523.5461 & -1523.6426 & 0 \\
\hline TSI & -1523.5384 & -1523.6323 & 1 \\
\hline II & -1523.5659 & -1523.6642 & 0 \\
\hline III & -1294.7470 & -1294.8327 & 0 \\
\hline IV & -1635.5394 & -1635.6333 & 0 \\
\hline TSIV & -1635.5232 & -1635.6146 & 1 \\
\hline v & -1635.5529 & -1635.6452 & 0 \\
\hline VI & -1864.3675 & -1864.4698 & 0 \\
\hline tsVI & -1864.3217 & -1864.4239 & 1 \\
\hline VII & -1864.3839 & -1864.4892 & 0 \\
\hline tsVII & -1864.3411 & -1864.4467 & 1 \\
\hline VIII & -1864.3706 & -1864.4774 & 0 \\
\hline IV-2 & -1635.5356 & -1635.6304 & 0 \\
\hline TSIV-2 & -1635.5119 & -1635.6056 & 1 \\
\hline IV-3 & -1635.5496 & -1635.6431 & 0 \\
\hline tsIV-3 & -1635.5463 & -1635.6389 & 1 \\
\hline$V-2$ & -1635.5460 & -1635.6414 & 0 \\
\hline VI-2 & -1864.3601 & -1864.4668 & 0 \\
\hline
\end{tabular}


Table S3. Summary of the calculation conducted at the $\omega B 97 X-D / 6-311+G(d, p) \& S D D / P C M(D C E) / / \omega B 97 X-D / 6-31 G+(d, p) \& L a n L 2 D Z / P C M(D C E)$ level.

\begin{tabular}{|l|l|l|l|}
\hline & $\begin{array}{l}\text { EE }+ \text { Thermal Enthalpy } \\
\text { Correction [Hartree] }\end{array}$ & $\begin{array}{l}\text { EE }+ \text { Thermal Free Energy } \\
\text { Correction [Hartree] }\end{array}$ & $\begin{array}{l}\text { Number of Imaginary } \\
\text { Frequency }\end{array}$ \\
\hline AcOH & -229.0259 & -229.0590 & 0 \\
\hline carbonate & -341.1075 & -341.1402 & 0 \\
\hline product & -496.8498 & -496.8909 & 0 \\
\hline PreA & -1526.0675 & -1526.1622 & 0 \\
\hline tsA & -1526.0565 & -1526.1479 & 1 \\
\hline intA & -1526.0797 & -1526.1737 & 0 \\
\hline intB & -1297.0283 & -1297.1108 & 0 \\
\hline intC & -1638.1614 & -1638.2536 & 0 \\
\hline tsCD & -1638.1440 & -1638.2340 & 1 \\
\hline intD & -1638.1787 & -1638.2691 & 0 \\
\hline intE & -1867.2267 & -1867.3287 & 0 \\
\hline tsEF & -1867.1742 & -1867.2745 & 1 \\
\hline intF & -1867.2365 & -1867.3393 & 0 \\
\hline tsFG & -1867.2022 & -1867.3053 & 1 \\
\hline intG & -1867.2343 & -1867.3391 & 0 \\
\hline intH & -1711.4877 & -1711.5844 & 0 \\
\hline & & & \\
\hline
\end{tabular}




\section{Copy of NMR Spectra}

${ }^{1} \mathrm{H}$ NMR of 3a $\left(400 \mathrm{MHz}, \mathrm{CDCl}_{3}\right)$

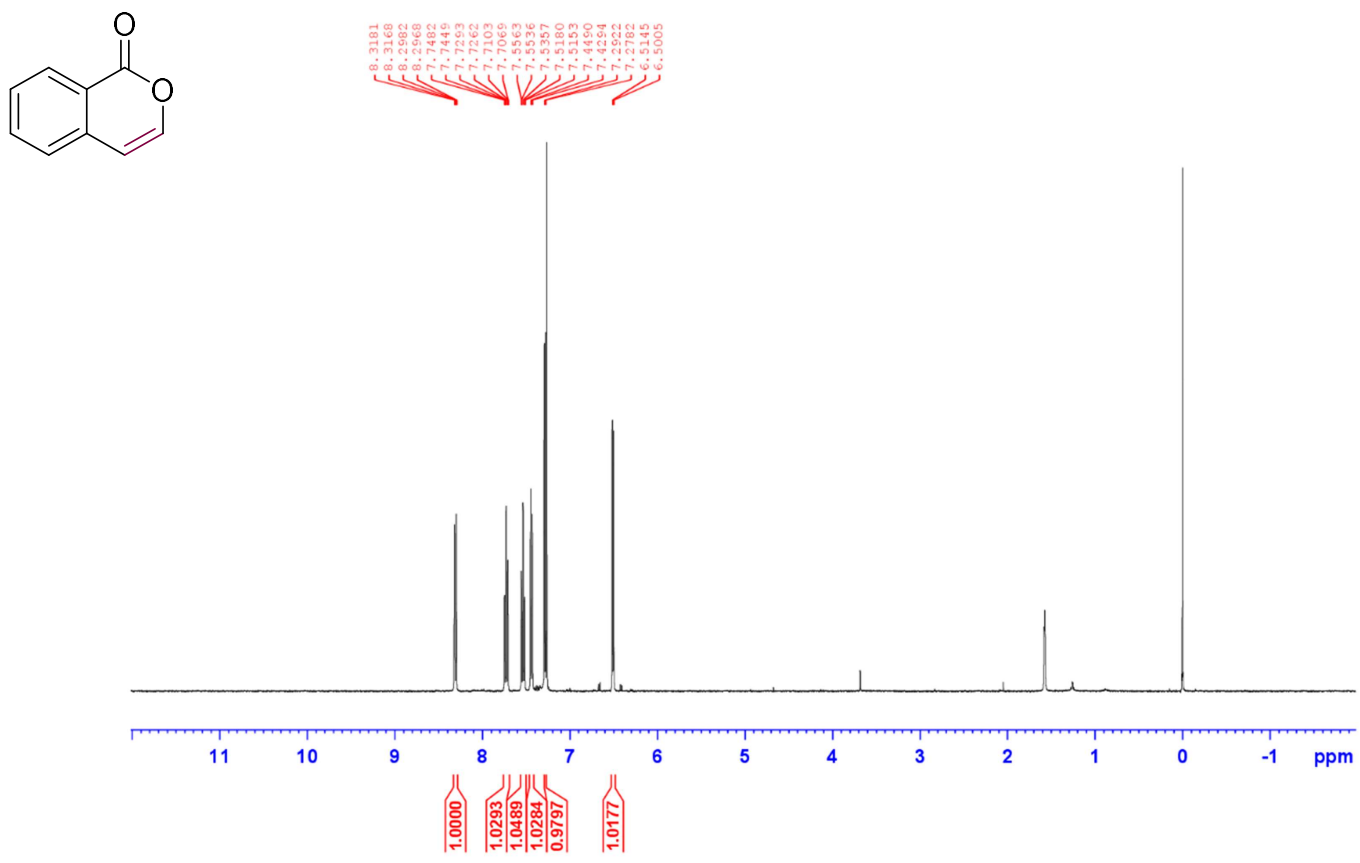

${ }^{13} \mathrm{C} \mathrm{NMR}$ of $\mathbf{3 a}\left(100 \mathrm{MHz}, \mathrm{CDCl}_{3}\right)$<smiles>O=c1occc2ccccc12</smiles>

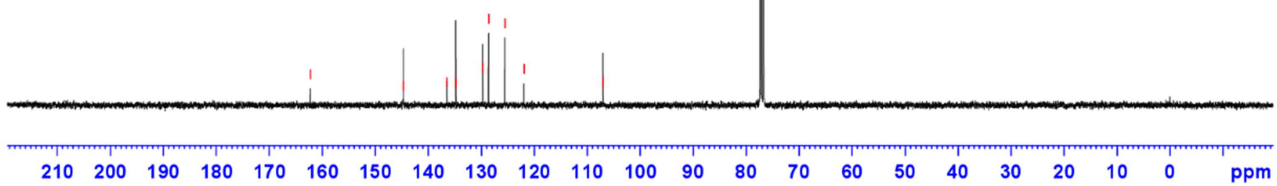


${ }^{1} \mathrm{H}$ NMR of $\mathbf{3 b}$ (400 MHz, acetone- $d_{6}$ )<smiles>Cc1ccc2c(=O)occc2c1</smiles>

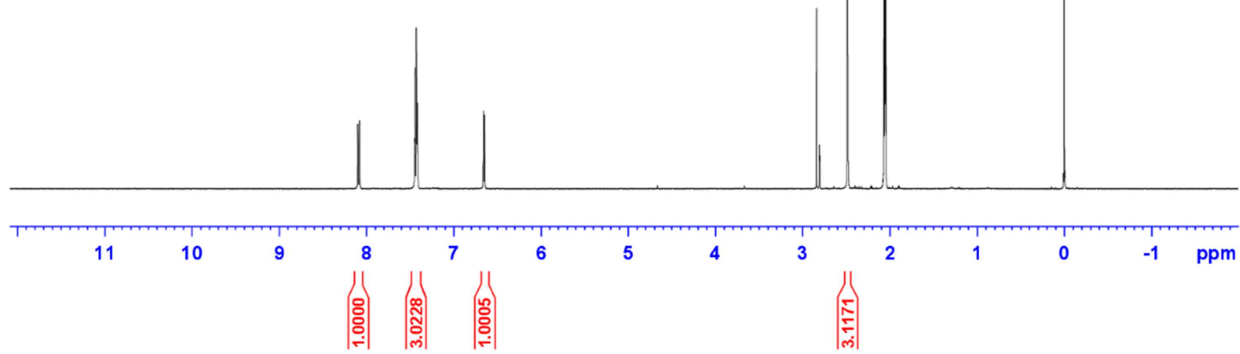

${ }^{13} \mathrm{C}$ NMR of $3 \mathbf{b}\left(100 \mathrm{MHz}\right.$, acetone- $\left.d_{6}\right)$<smiles>Cc1ccc2c(=O)occc2c1</smiles>

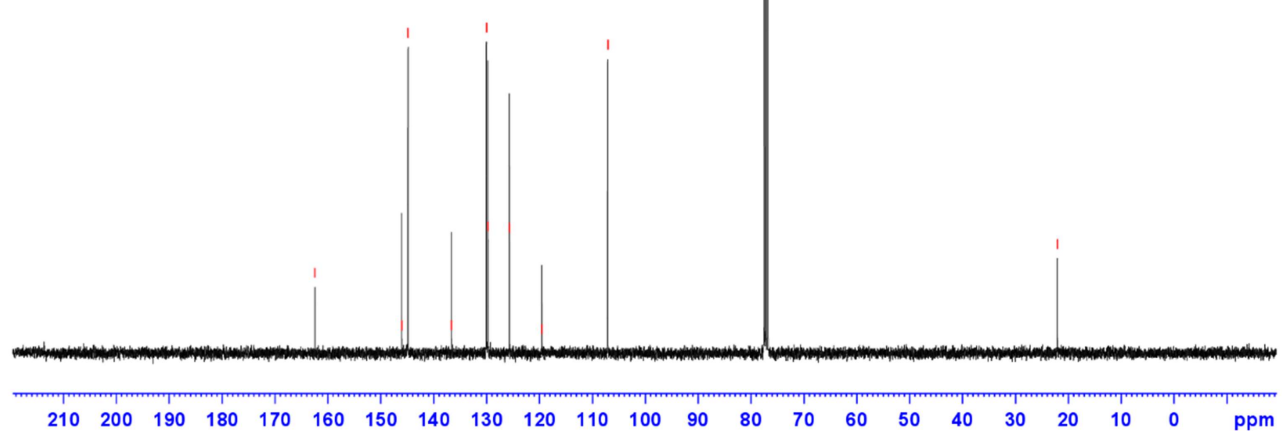


${ }^{1} \mathrm{H}$ NMR of $3 \mathbf{c}\left(400 \mathrm{MHz}\right.$, acetone- $d_{6}$ )<smiles>COc1ccc2c(c1)C=CCC2C=O</smiles>

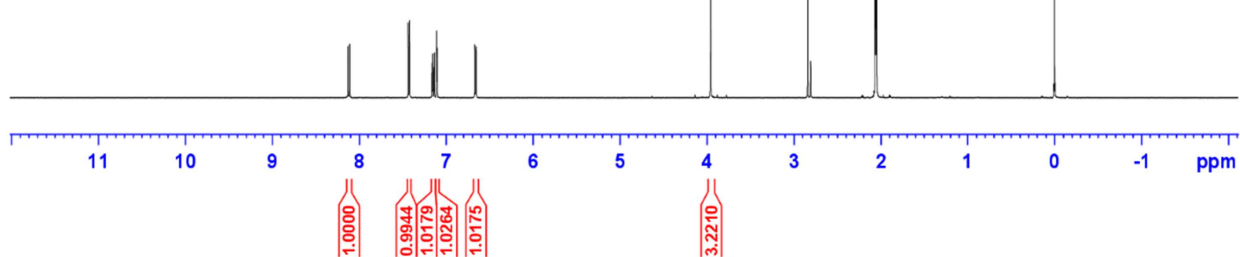

${ }^{13} \mathrm{C}$ NMR of $3 \mathbf{c}\left(100 \mathrm{MHz}\right.$, acetone- $\left.d_{6}\right)$<smiles>COc1ccc2c(=O)occc2c1</smiles>

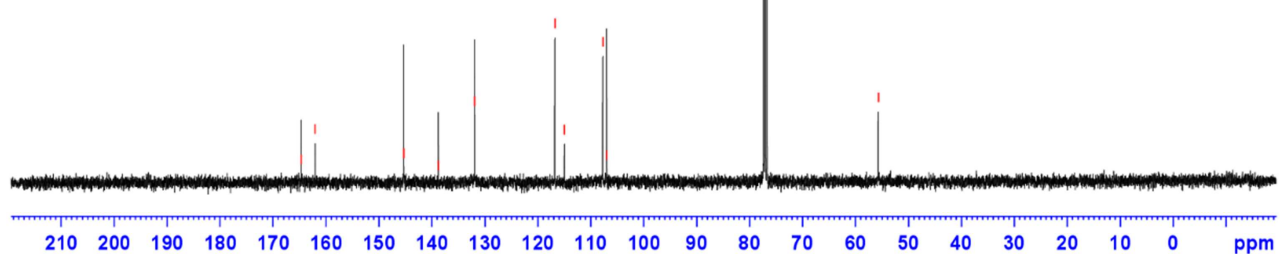


${ }^{1} \mathrm{H}$ NMR of 3d (400 MHz, DMSO- $d_{6}$ )

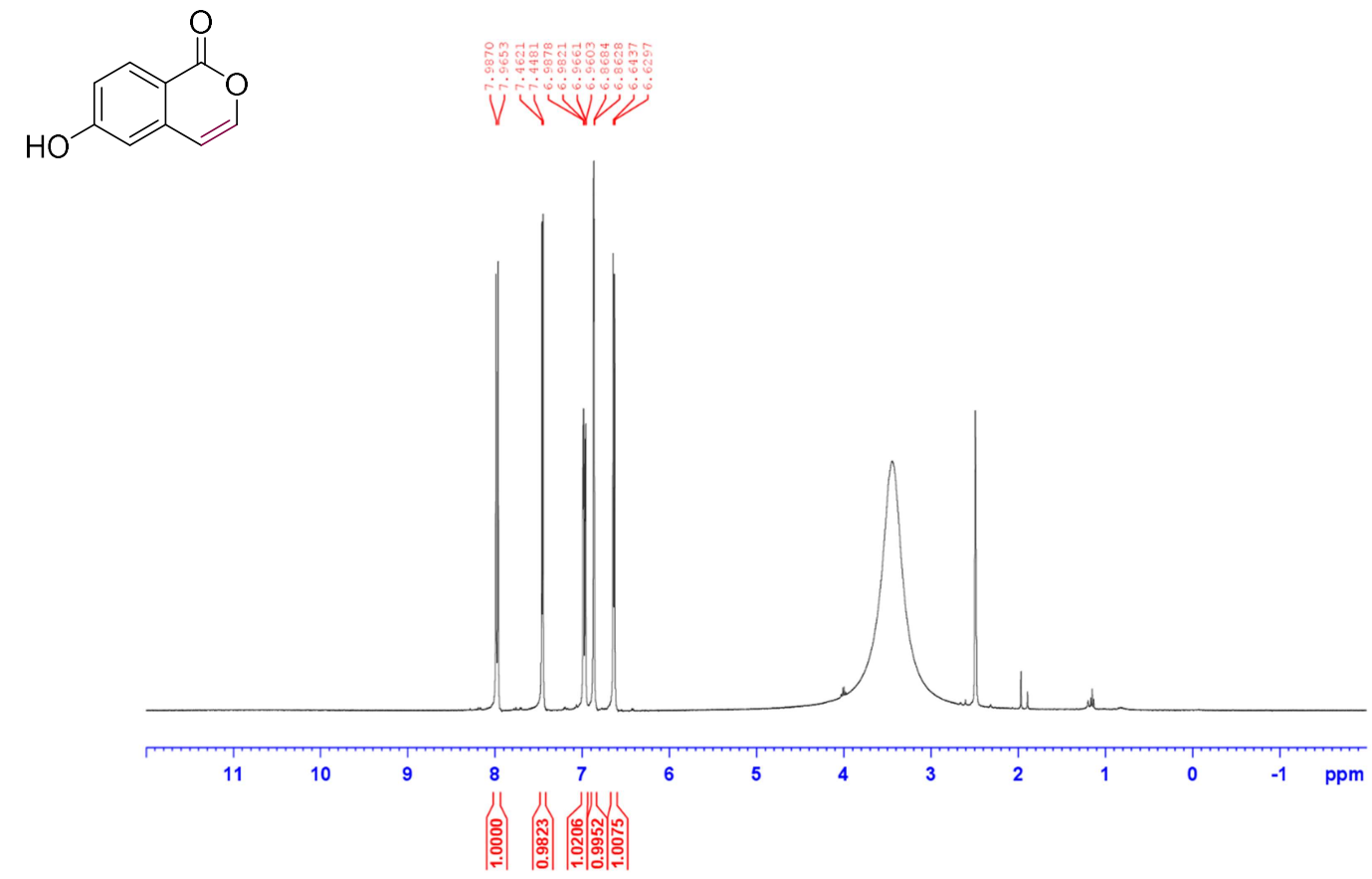

${ }^{13} \mathrm{C}$ NMR of 3d (100 MHz, DMSO- $d_{6}$ )<smiles>O=c1occc2cc(O)ccc12</smiles>

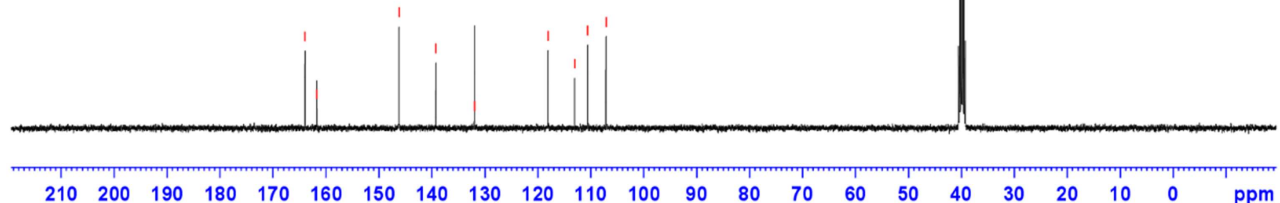


${ }^{1} \mathrm{H}$ NMR of $3 \mathbf{e}\left(400 \mathrm{MHz}, \mathrm{CDCl}_{3}\right)$<smiles>O=c1occc2cc(Cl)ccc12</smiles><smiles>CC(C)C</smiles>

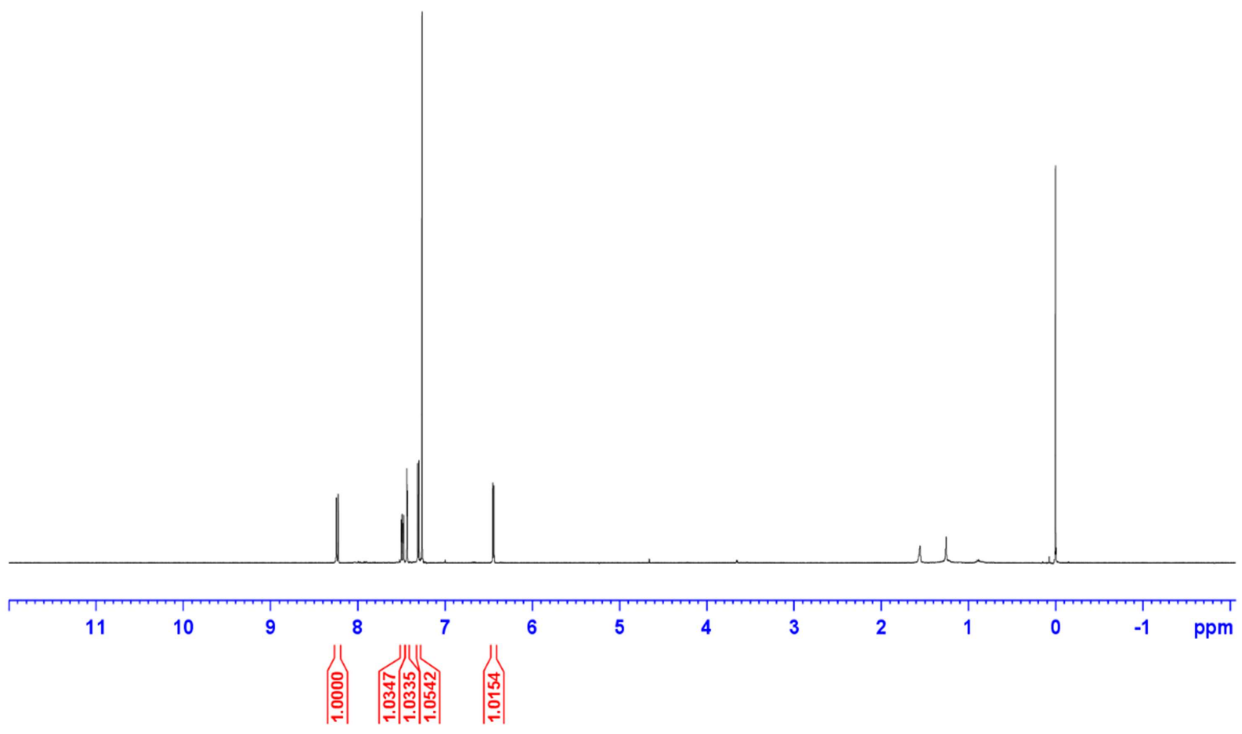

${ }^{13} \mathrm{C}$ NMR of $3 \mathbf{e}\left(100 \mathrm{MHz}, \mathrm{CDCl}_{3}\right)$<smiles>O=c1occc2cc(Cl)ccc12</smiles>

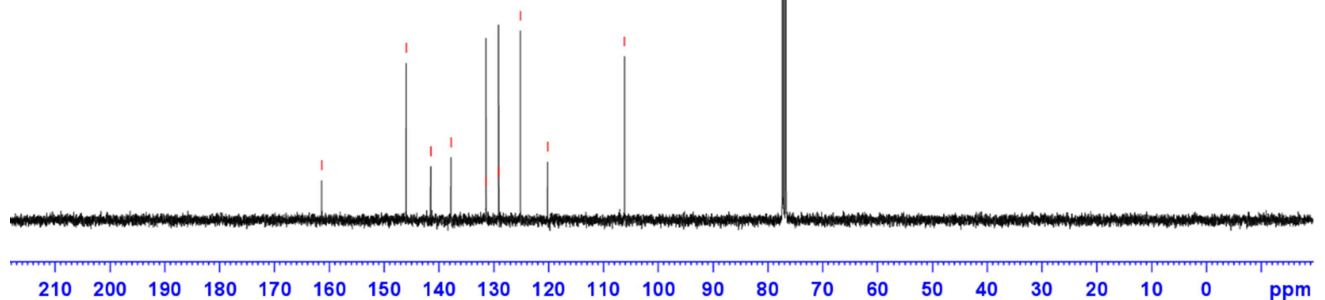


${ }^{1} \mathrm{H}$ NMR of $\mathbf{3 f}\left(400 \mathrm{MHz}, \mathrm{CDCl}_{3}\right)$<smiles>O=C1CC=Cc2cc(Br)ccc21</smiles><smiles>C=CC=CC</smiles>

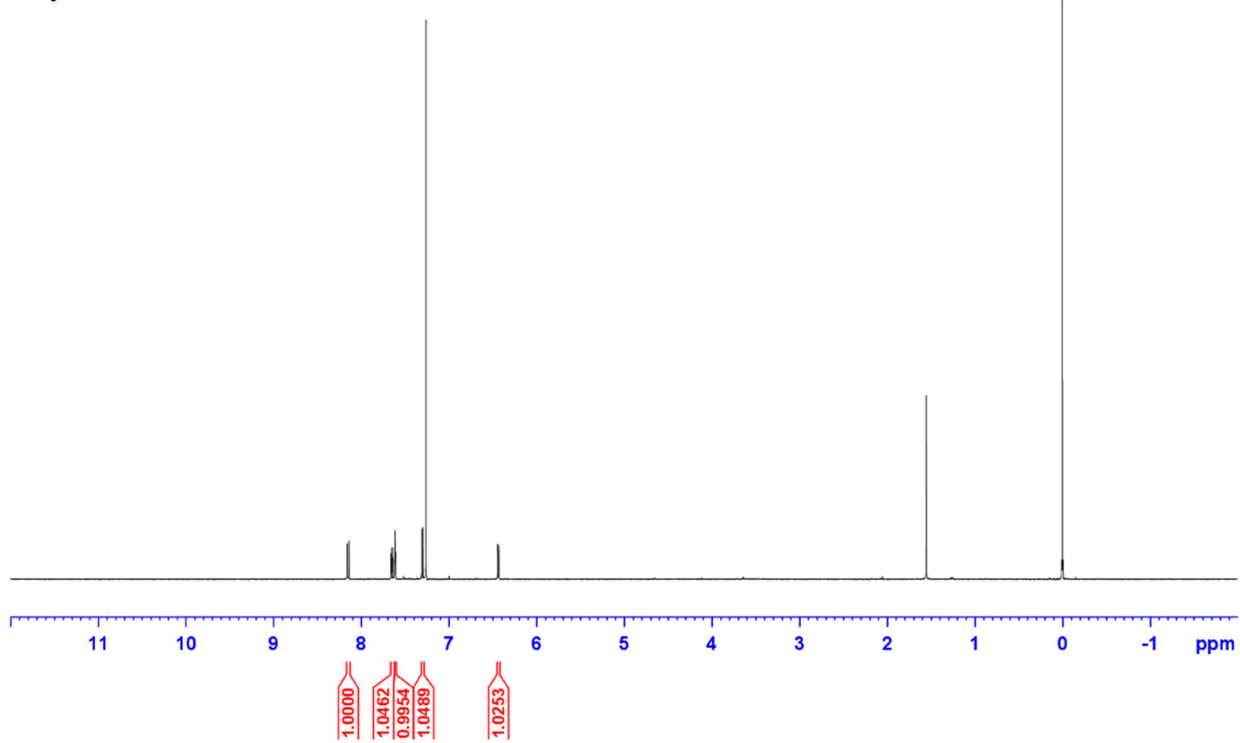

${ }^{13} \mathrm{C}$ NMR of $3 f\left(100 \mathrm{MHz}, \mathrm{CDCl}_{3}\right)$<smiles>O=c1occc2cc(Br)ccc12</smiles>

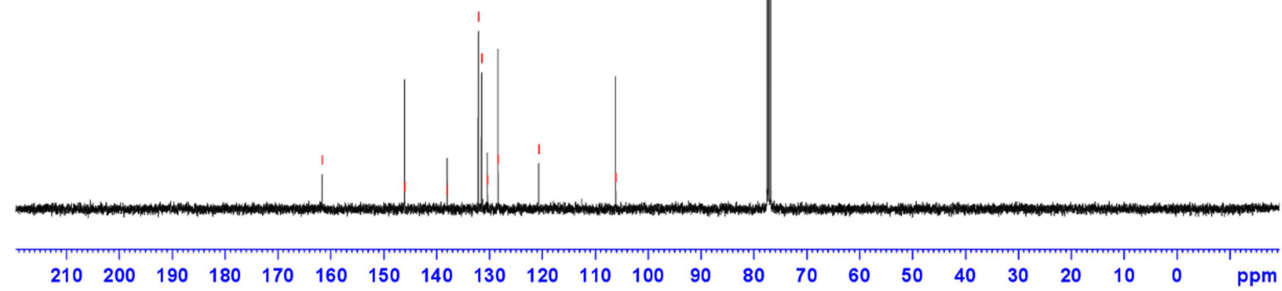


${ }^{1} \mathrm{H} \mathrm{NMR}$ of $\mathbf{3 g}\left(400 \mathrm{MHz}, \mathrm{CDCl}_{3}\right)$

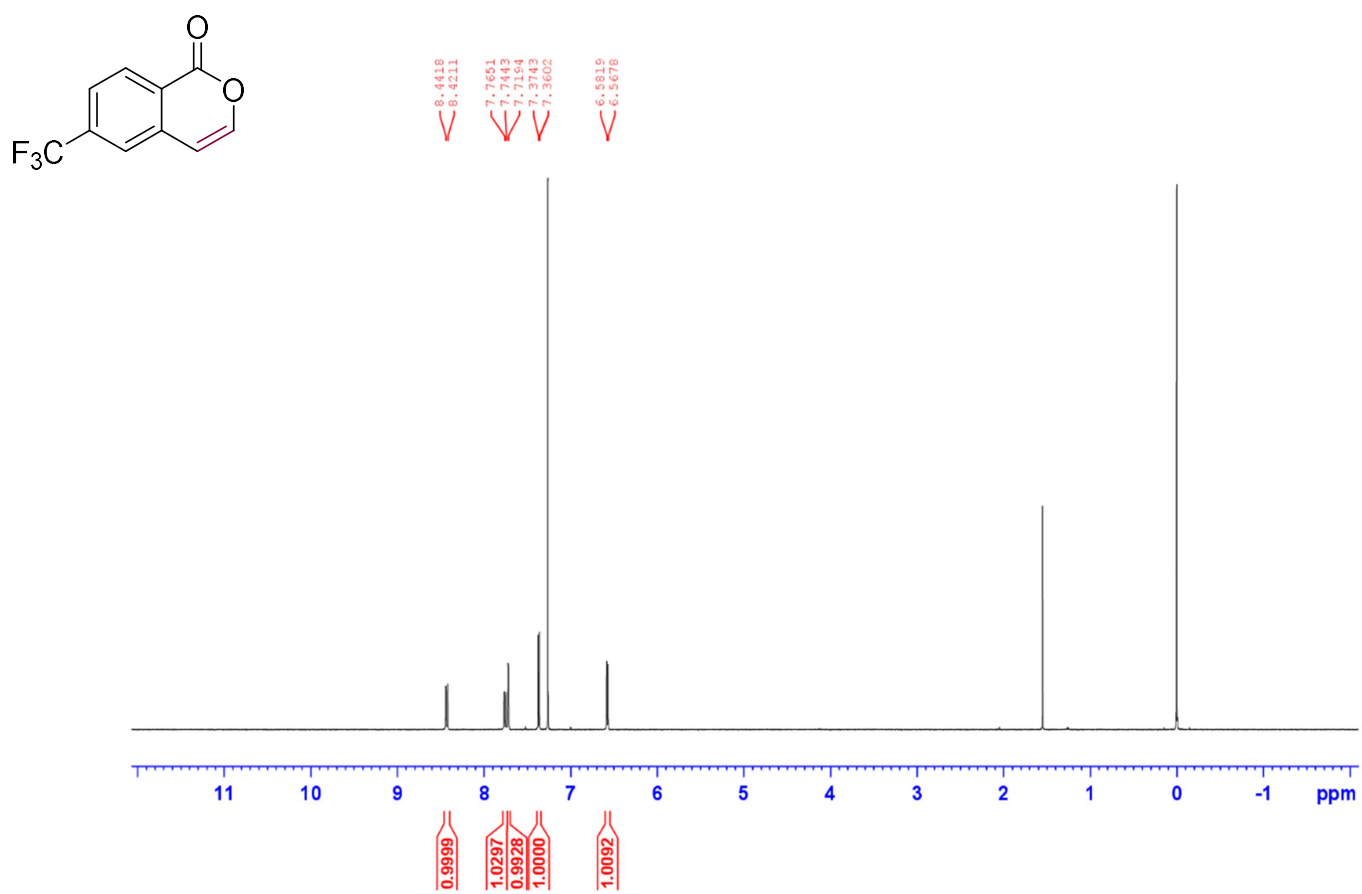

${ }^{13} \mathrm{C}$ NMR of $3 \mathbf{g}\left(100 \mathrm{MHz}, \mathrm{CDCl}_{3}\right)$<smiles>O=c1occc2cc(C(F)(F)F)ccc12</smiles>
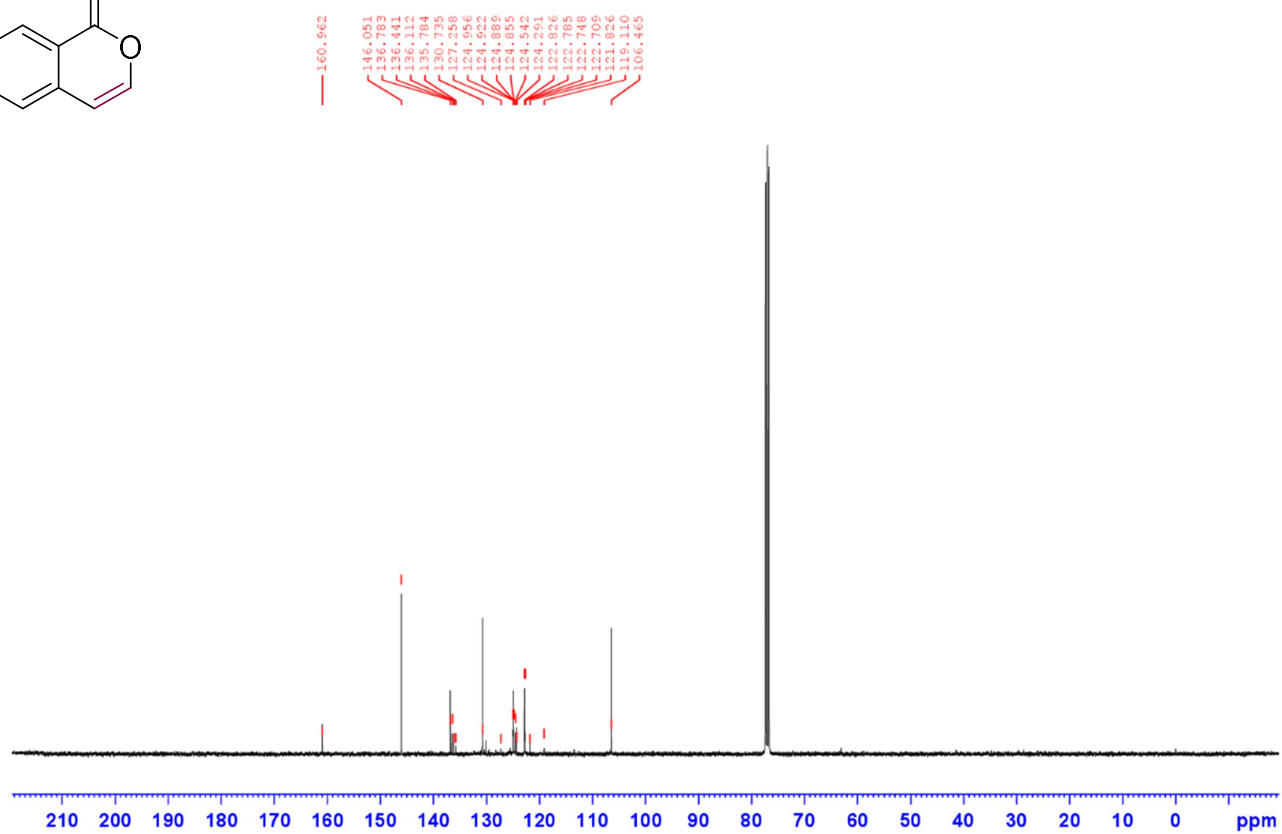
${ }^{19} \mathrm{~F}$ NMR of $\mathbf{3 g}\left(376 \mathrm{MHz}, \mathrm{CDCl}_{3}\right)$

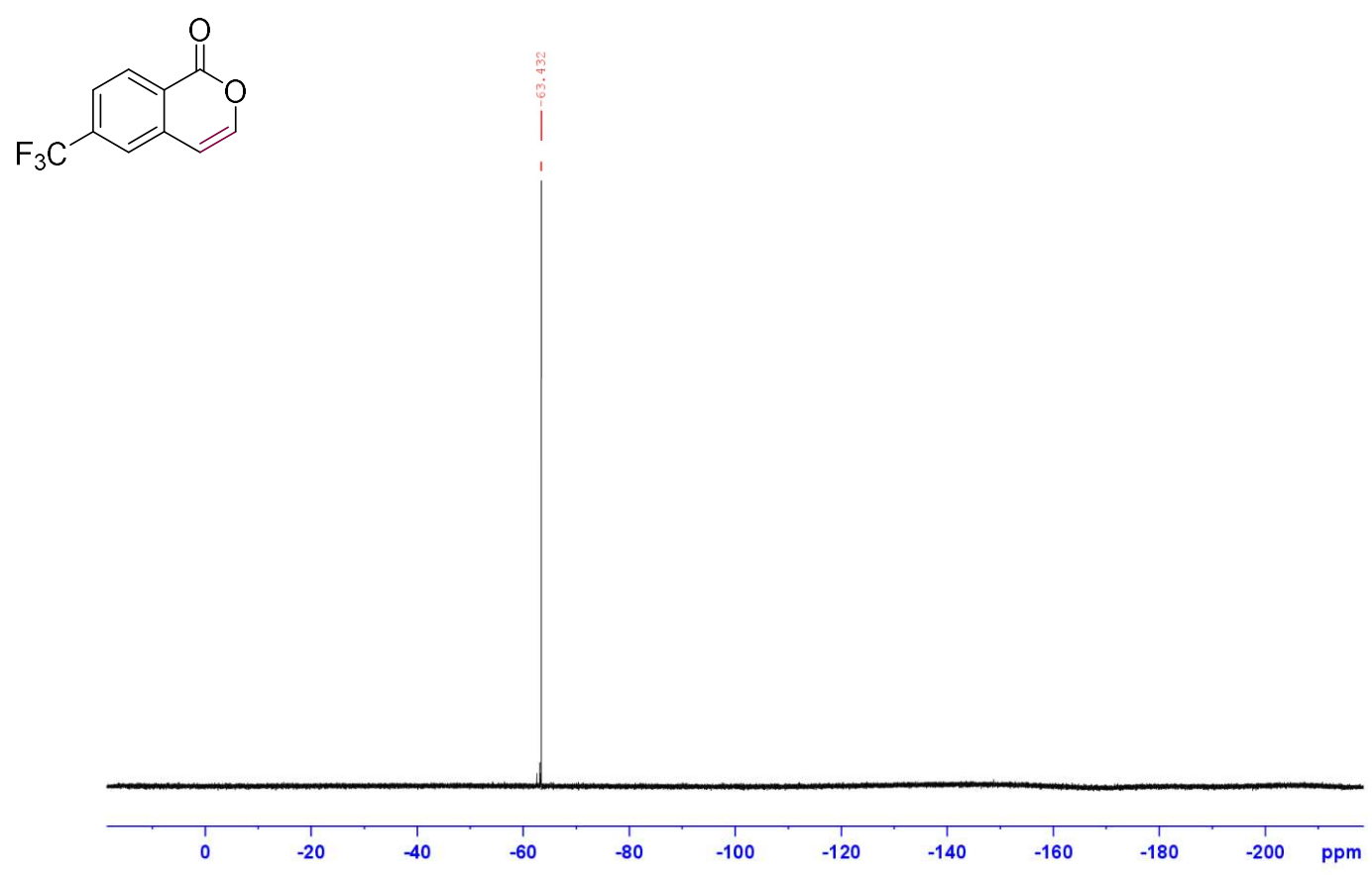


${ }^{1} \mathrm{H}$ NMR of $\mathbf{3 h}\left(400 \mathrm{MHz}, \mathrm{CDCl}_{3}\right)$<smiles>O=Cc1ccc2c(=O)occc2c1</smiles>

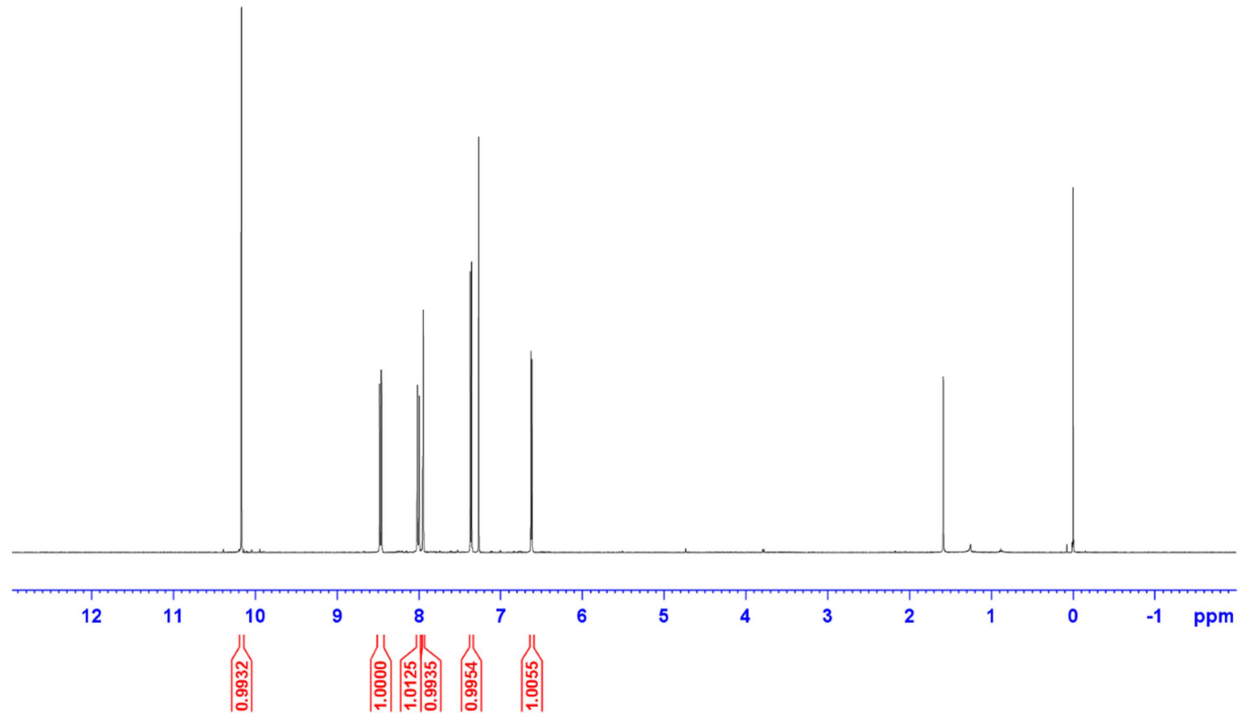

${ }^{13} \mathrm{C}$ NMR of $\mathbf{3 h}\left(100 \mathrm{MHz}, \mathrm{CDCl}_{3}\right)$<smiles>O=Cc1ccc2c(=O)occc2c1</smiles>

$\mathrm{OHC}$

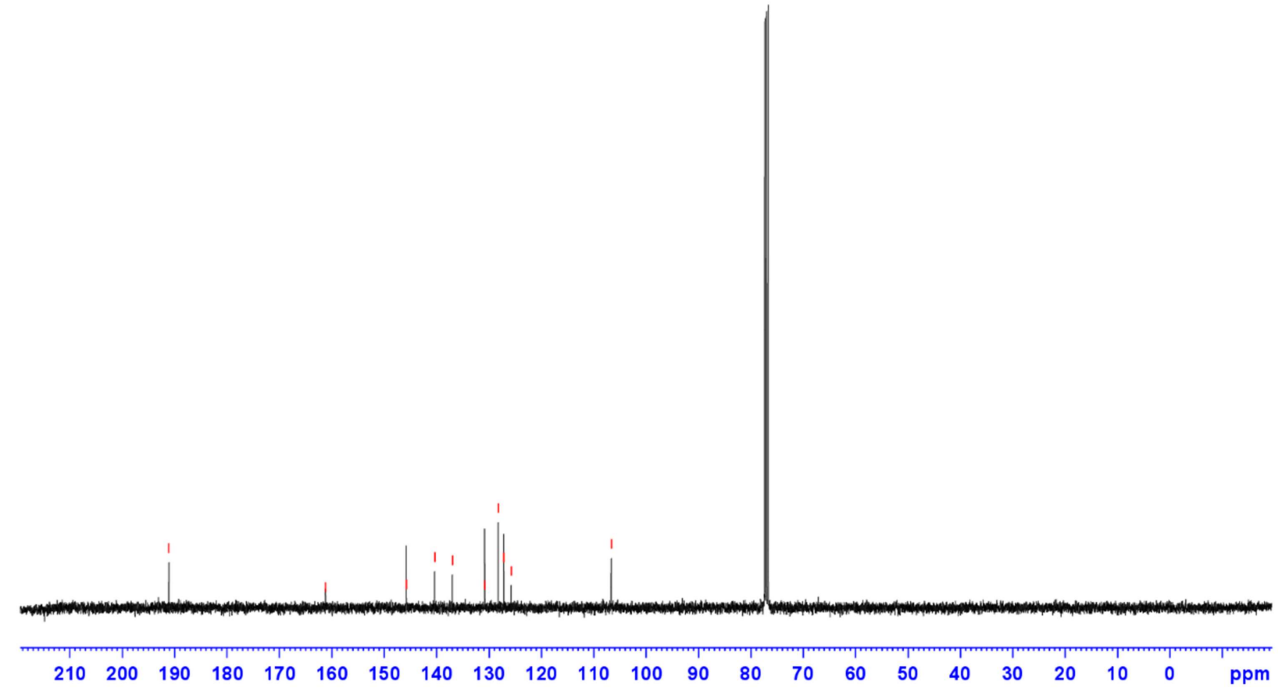


${ }^{1} \mathrm{H}$ NMR of $3 \mathbf{i}\left(400 \mathrm{MHz}, \mathrm{CDCl}_{3}\right)$

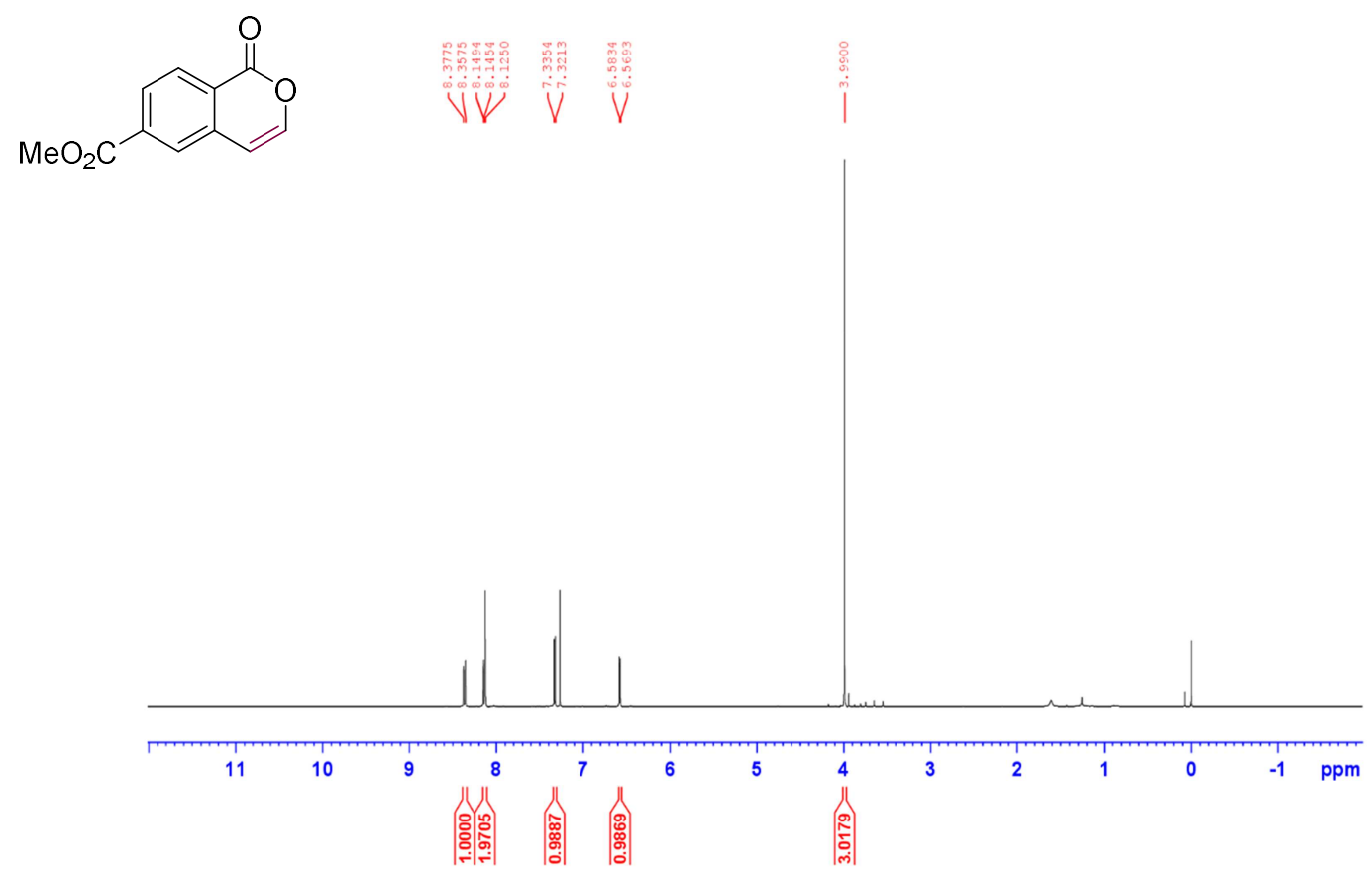

${ }^{13} \mathrm{C}$ NMR of $3 \mathbf{i}\left(100 \mathrm{MHz}, \mathrm{CDCl}_{3}\right)$
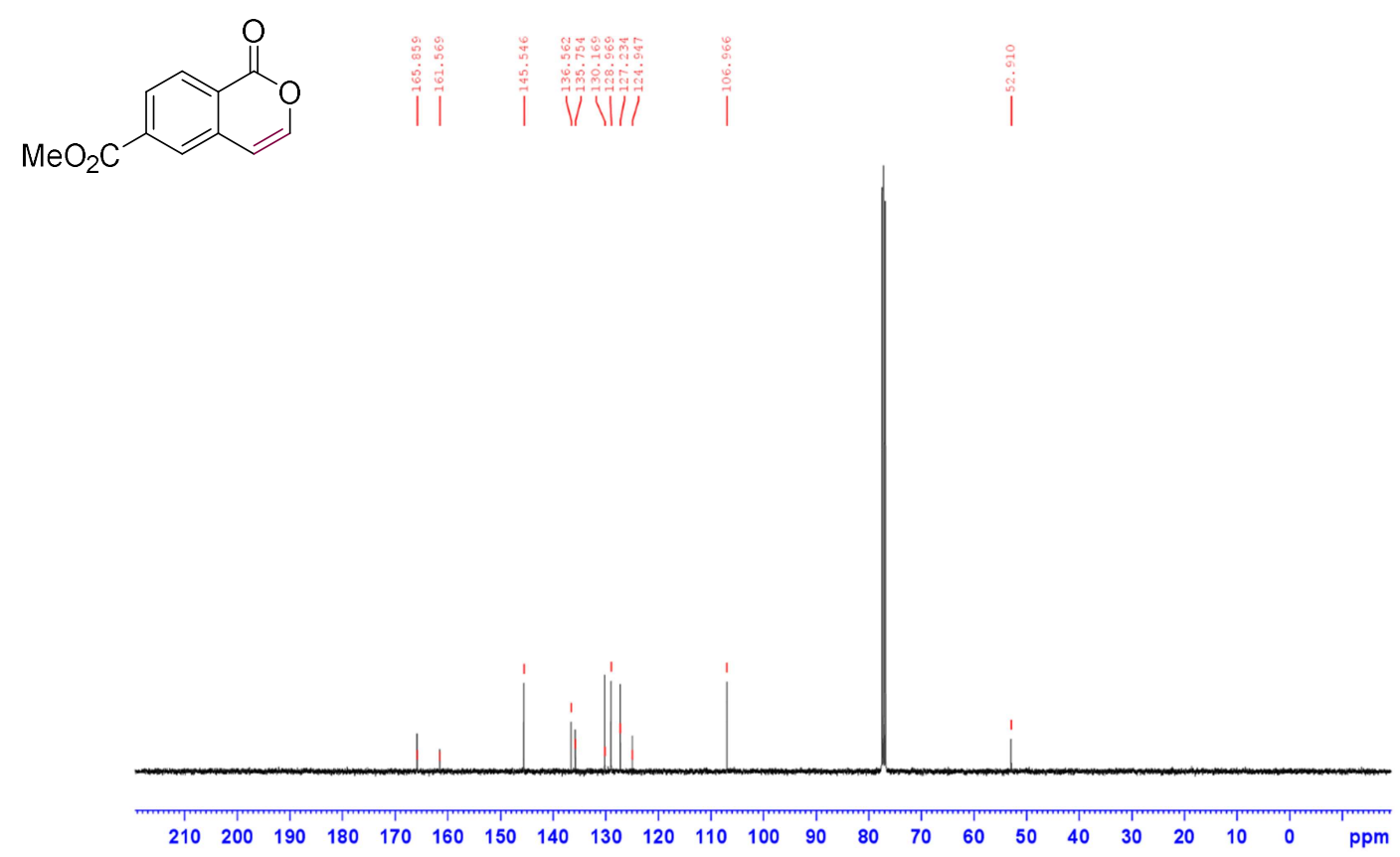
${ }^{1} \mathrm{H}$ NMR of $\mathbf{3 j}\left(400 \mathrm{MHz}, \mathrm{CDCl}_{3}\right.$ )

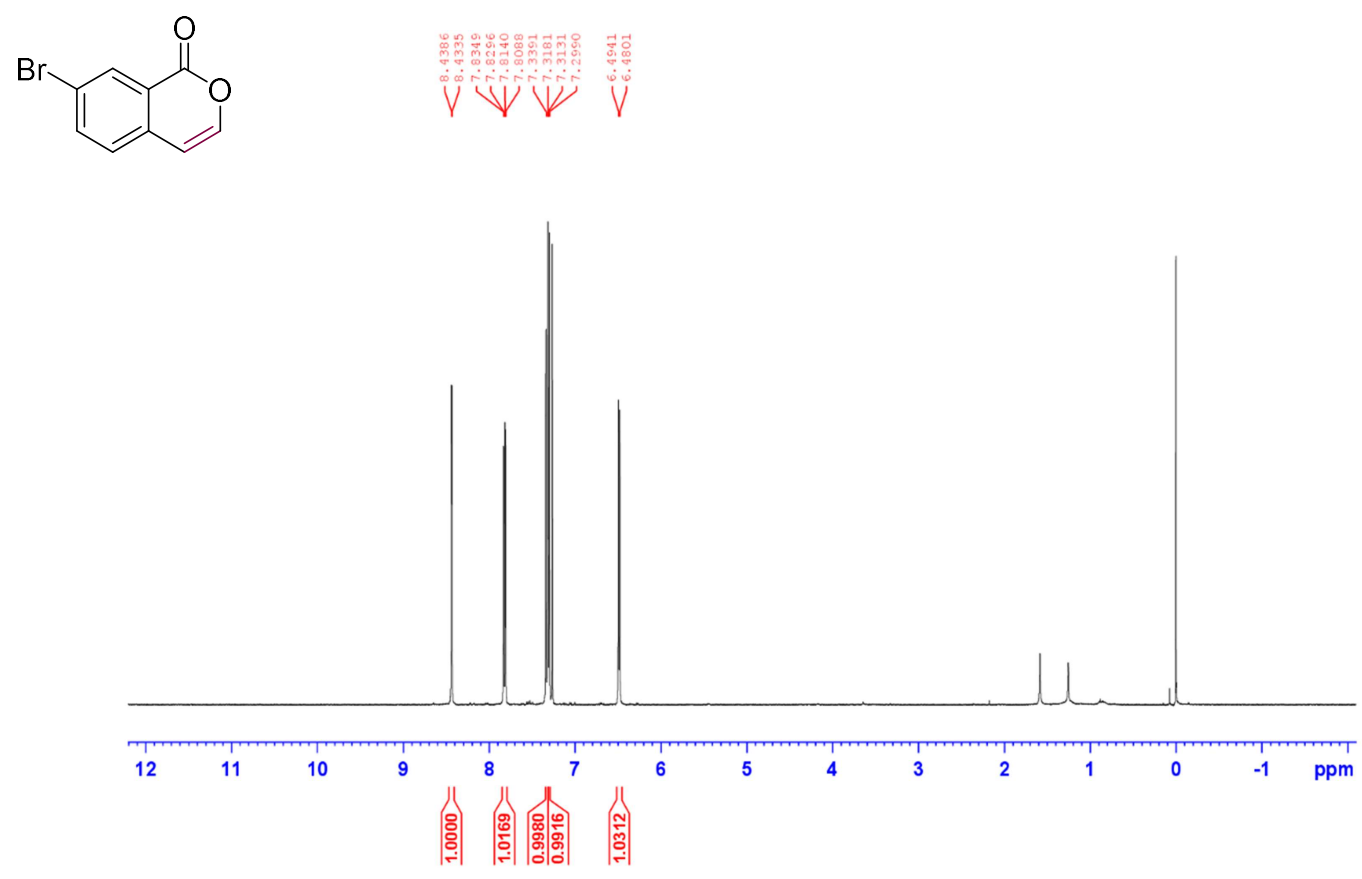

${ }^{13} \mathrm{C} \mathrm{NMR}$ of $\mathbf{3 j}\left(100 \mathrm{MHz}, \mathrm{CDCl}_{3}\right)$
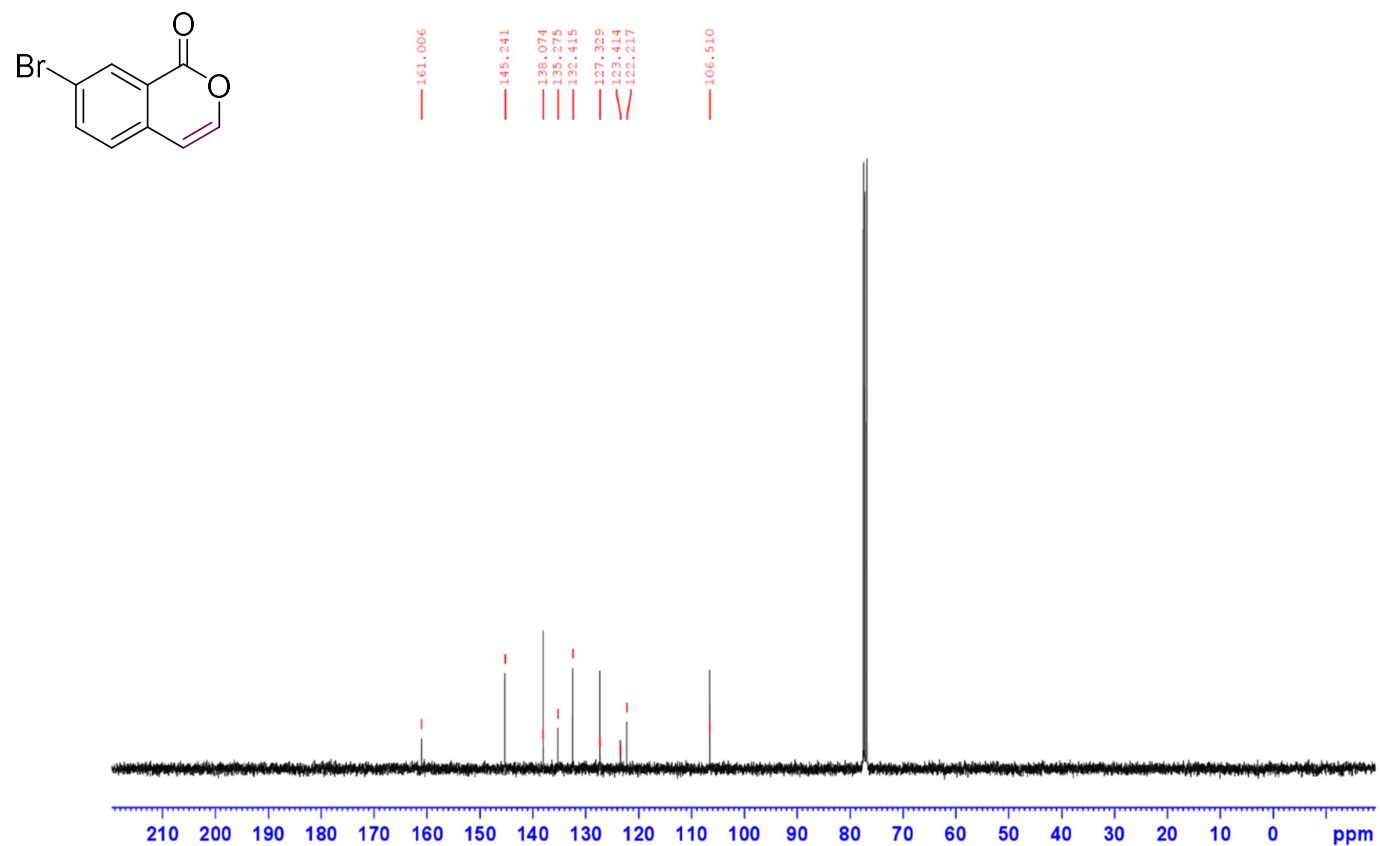
${ }^{1} \mathrm{H}$ NMR of $\mathbf{3 k}\left(400 \mathrm{MHz}, \mathrm{CDCl}_{3}\right)$

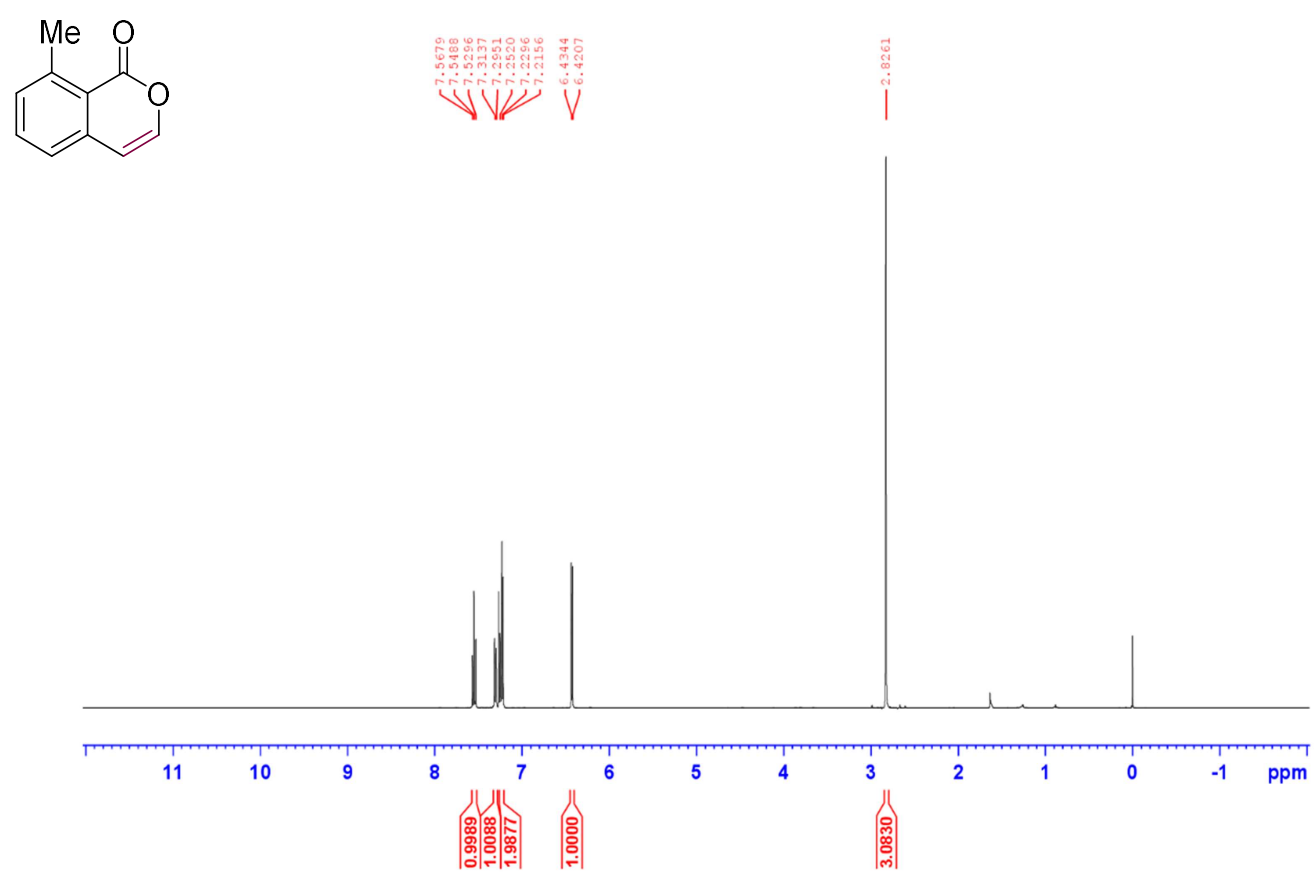

${ }^{13} \mathrm{C}$ NMR of $\mathbf{3 k}\left(100 \mathrm{MHz}, \mathrm{CDCl}_{3}\right)$

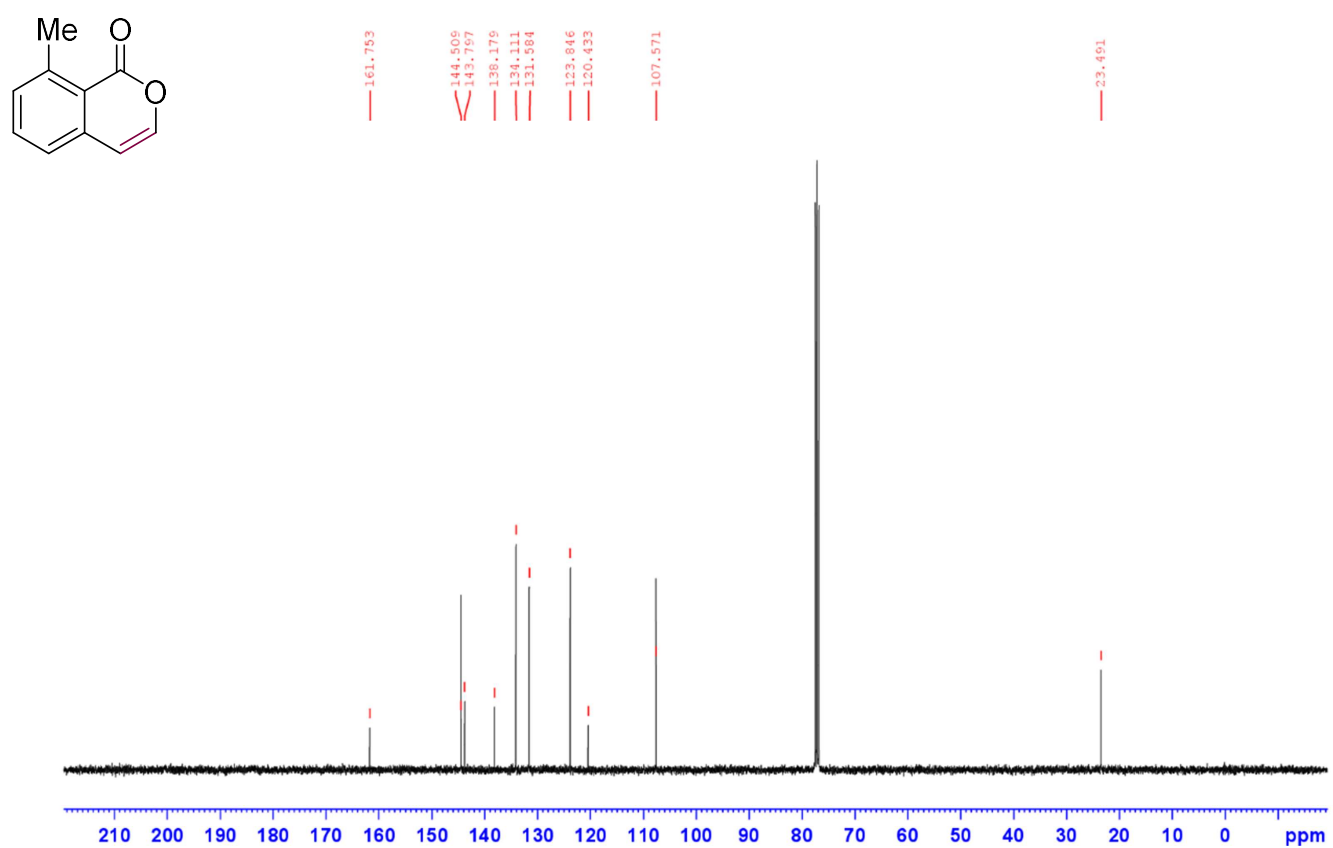


${ }^{1} \mathrm{H}$ NMR of $3 \mathbf{l}\left(400 \mathrm{MHz}, \mathrm{CDCl}_{3}\right)$

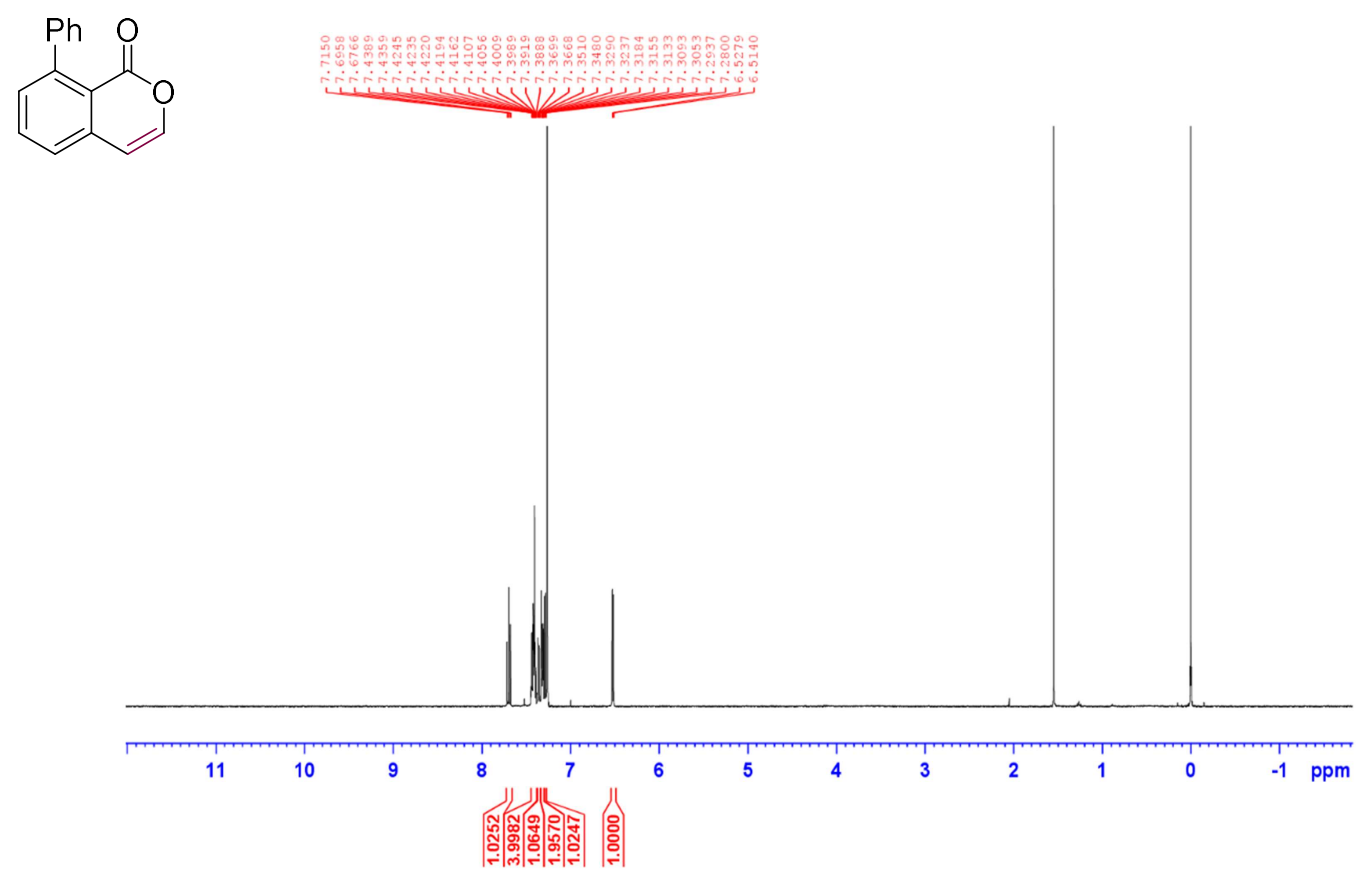

${ }^{13} \mathrm{C}$ NMR of $3 \mathbf{l}\left(100 \mathrm{MHz}, \mathrm{CDCl}_{3}\right)$
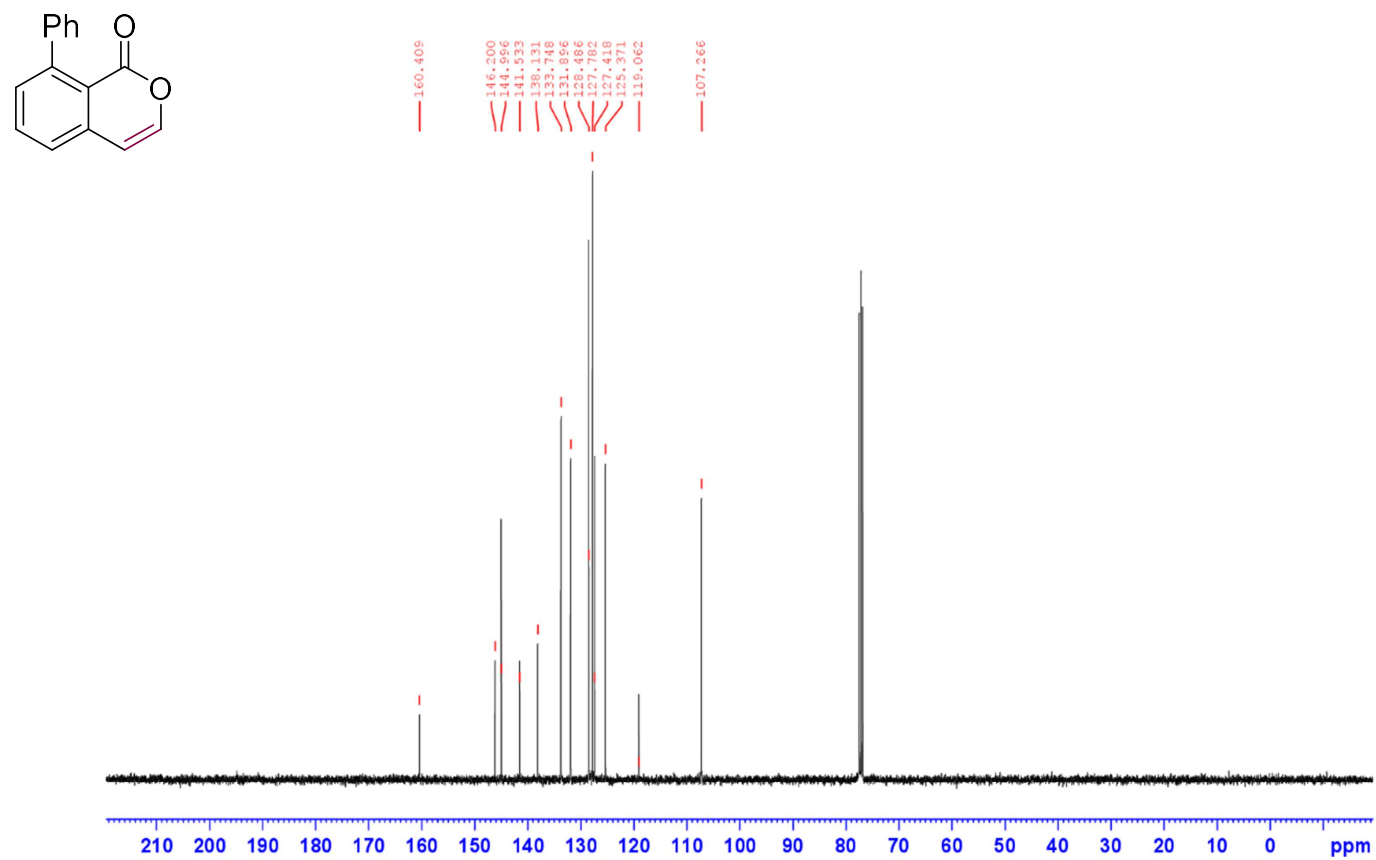
${ }^{1} \mathrm{H}$ NMR of $\mathbf{3 m}\left(400 \mathrm{MHz}, \mathrm{CDCl}_{3}\right)$

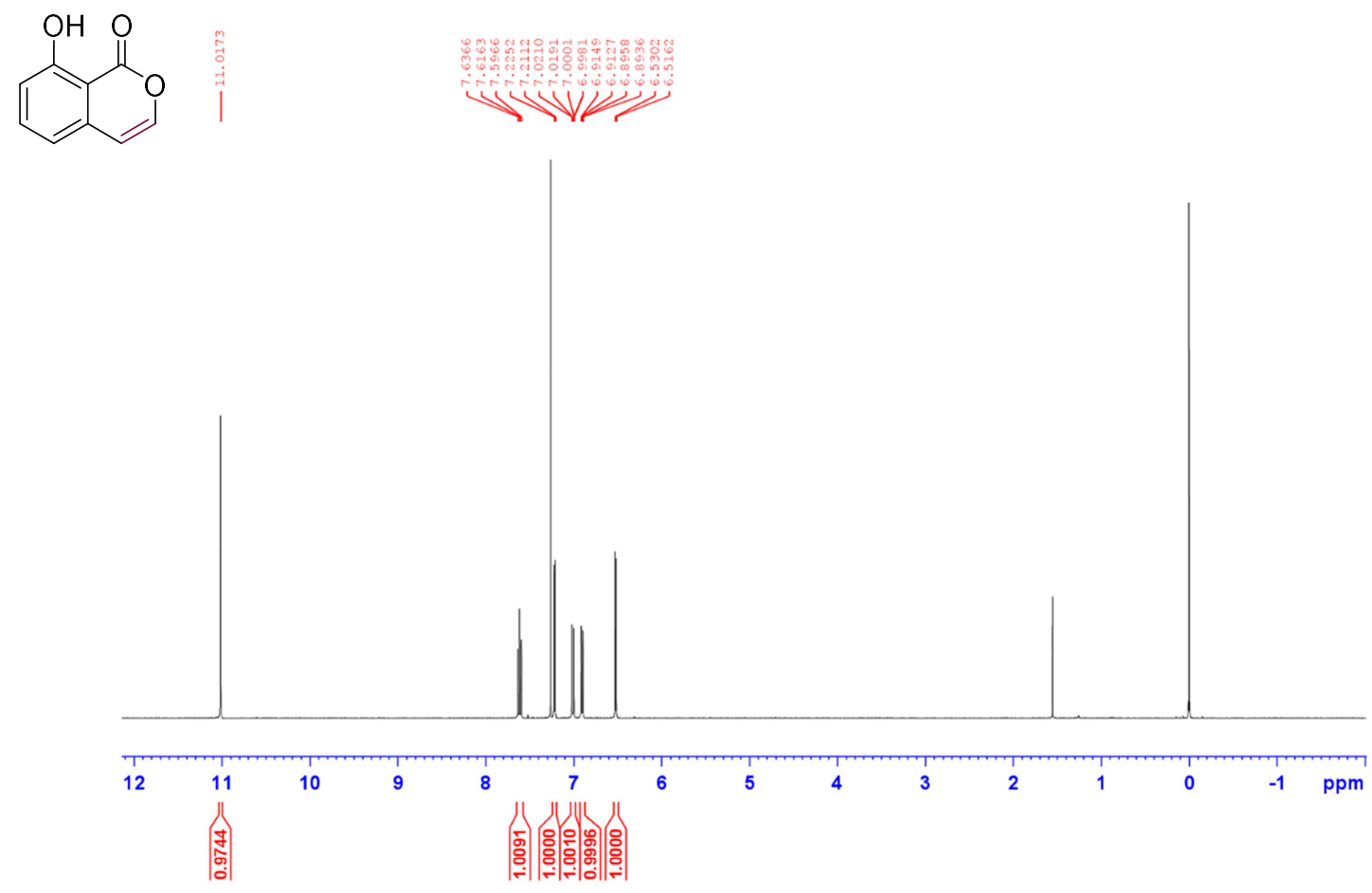

${ }^{13} \mathrm{C}$ NMR of $\mathbf{3 m}\left(100 \mathrm{MHz}, \mathrm{CDCl}_{3}\right)$
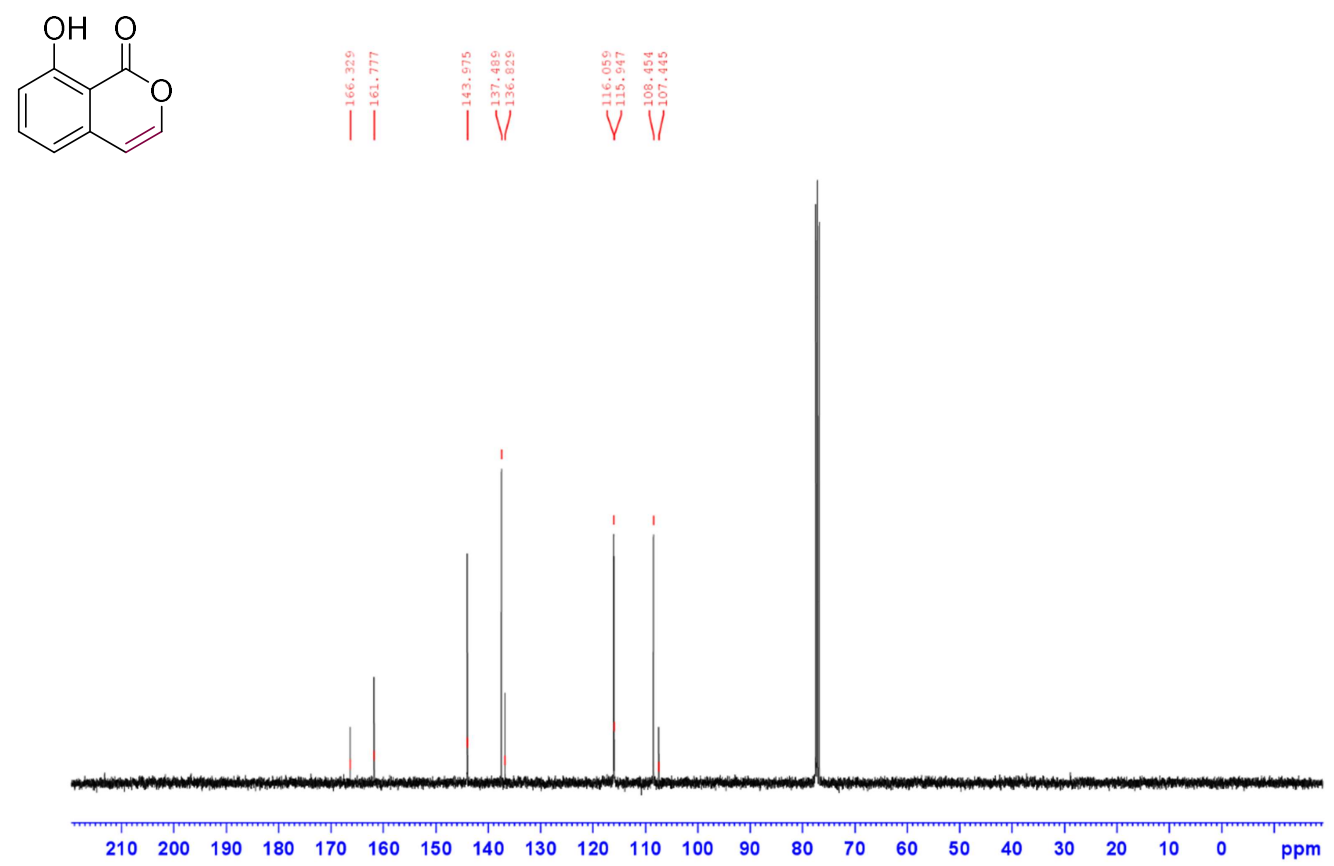
${ }^{1} \mathrm{H}$ NMR of $3 \mathbf{n}\left(400 \mathrm{MHz}, \mathrm{CDCl}_{3}\right)$

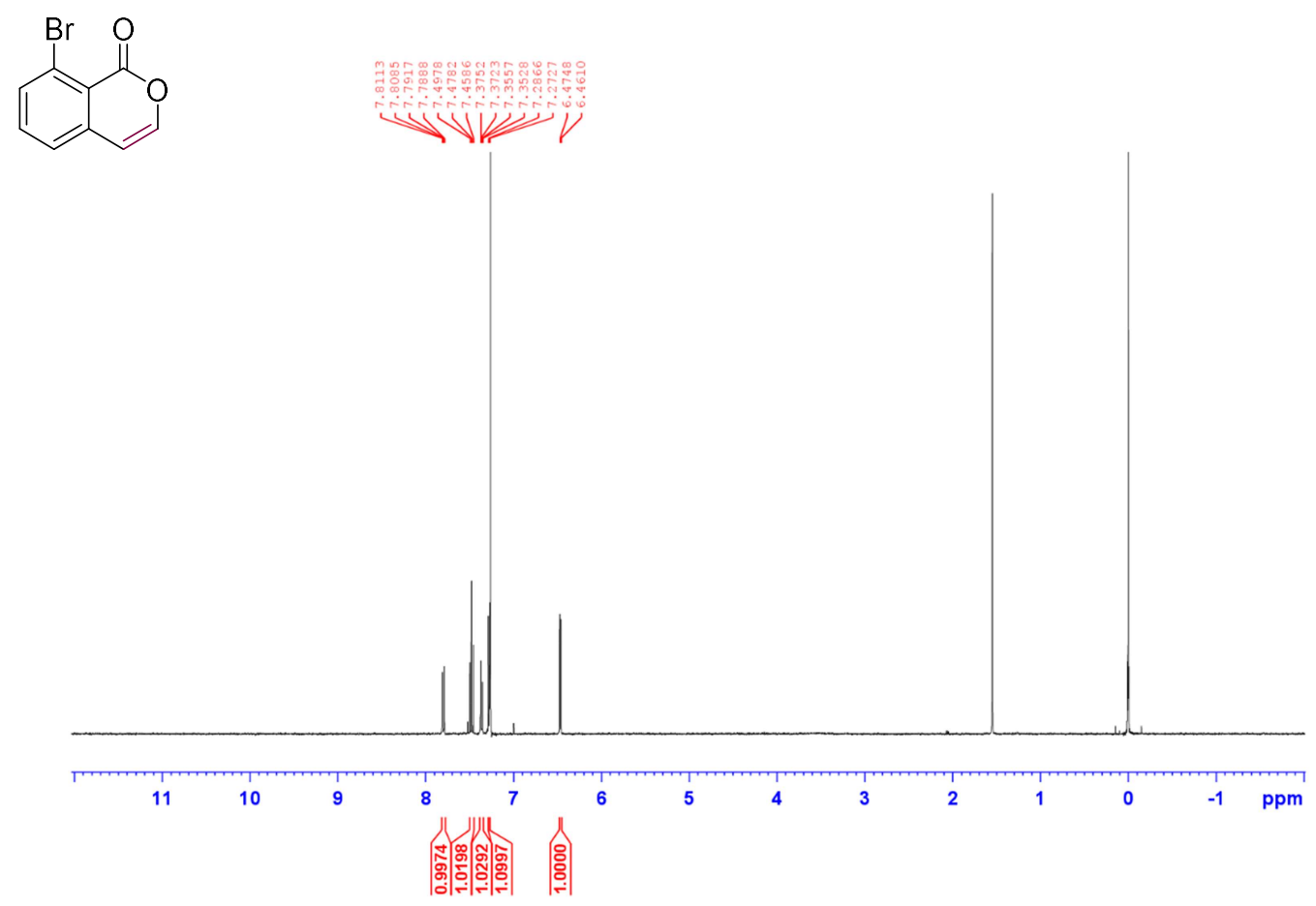

${ }^{13} \mathrm{C}$ NMR of 3 n $\left(100 \mathrm{MHz}, \mathrm{CDCl}_{3}\right)$<smiles>O=c1occc2cccc(Br)c12</smiles>

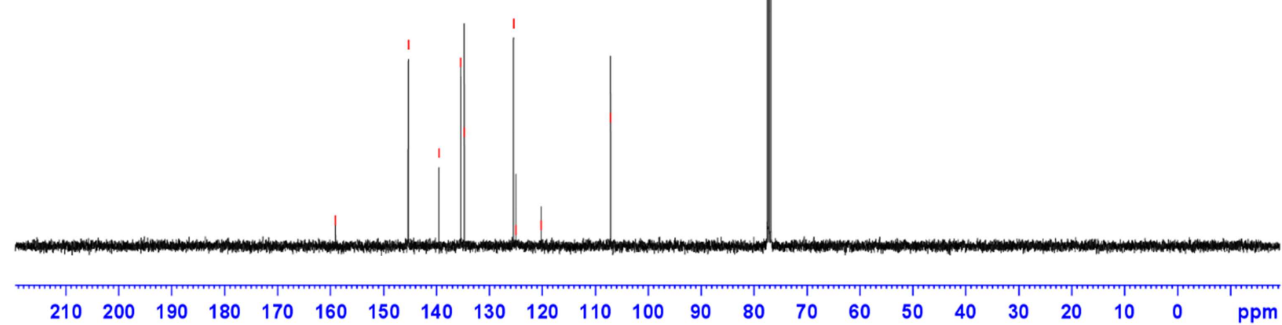


${ }^{1} \mathrm{H} \mathrm{NMR}$ of $3 \mathbf{0}\left(400 \mathrm{MHz}, \mathrm{CDCl}_{3}\right)$

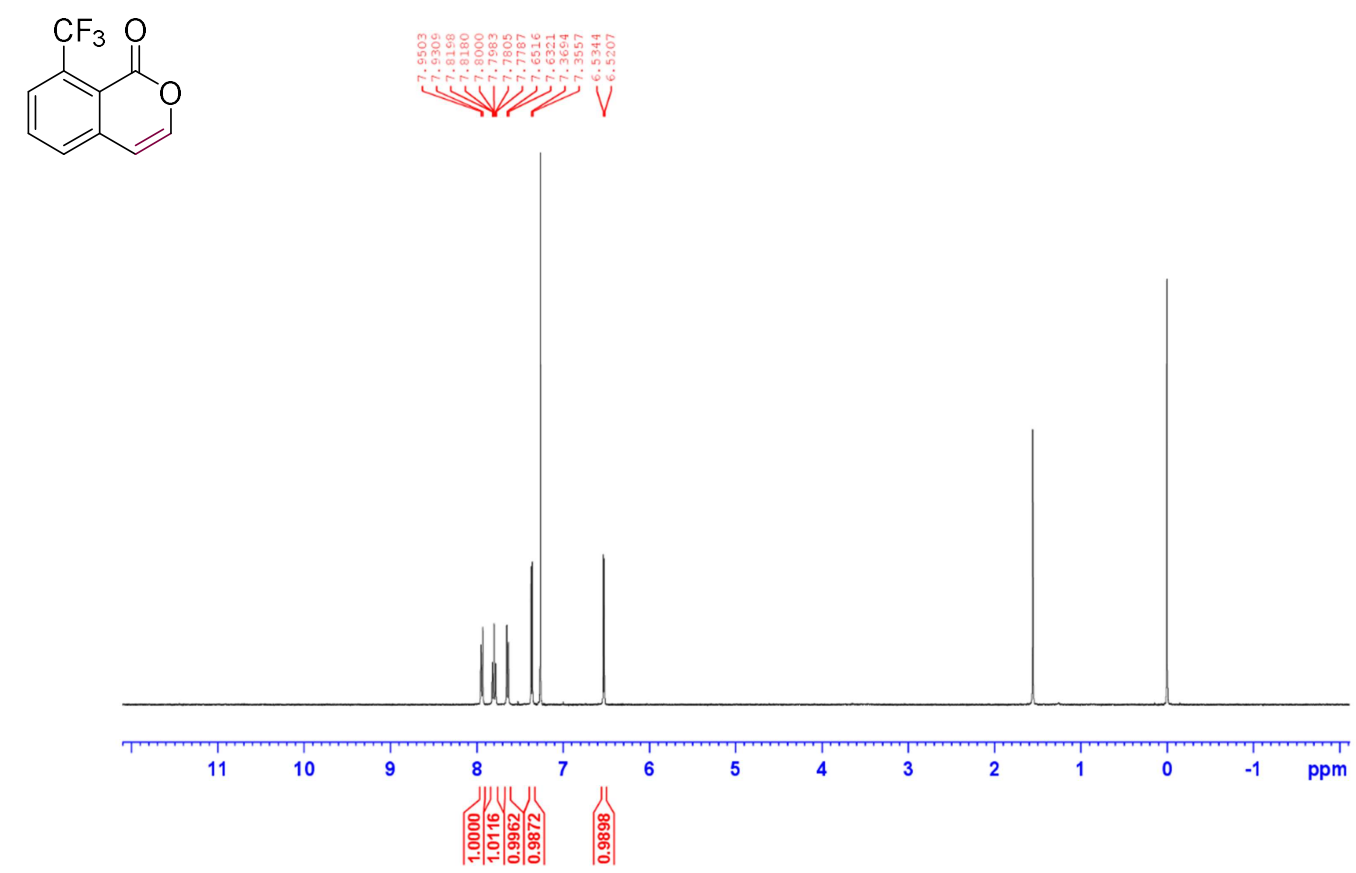

${ }^{13} \mathrm{C}$ NMR of $3 \mathbf{o}\left(100 \mathrm{MHz}, \mathrm{CDCl}_{3}\right)$

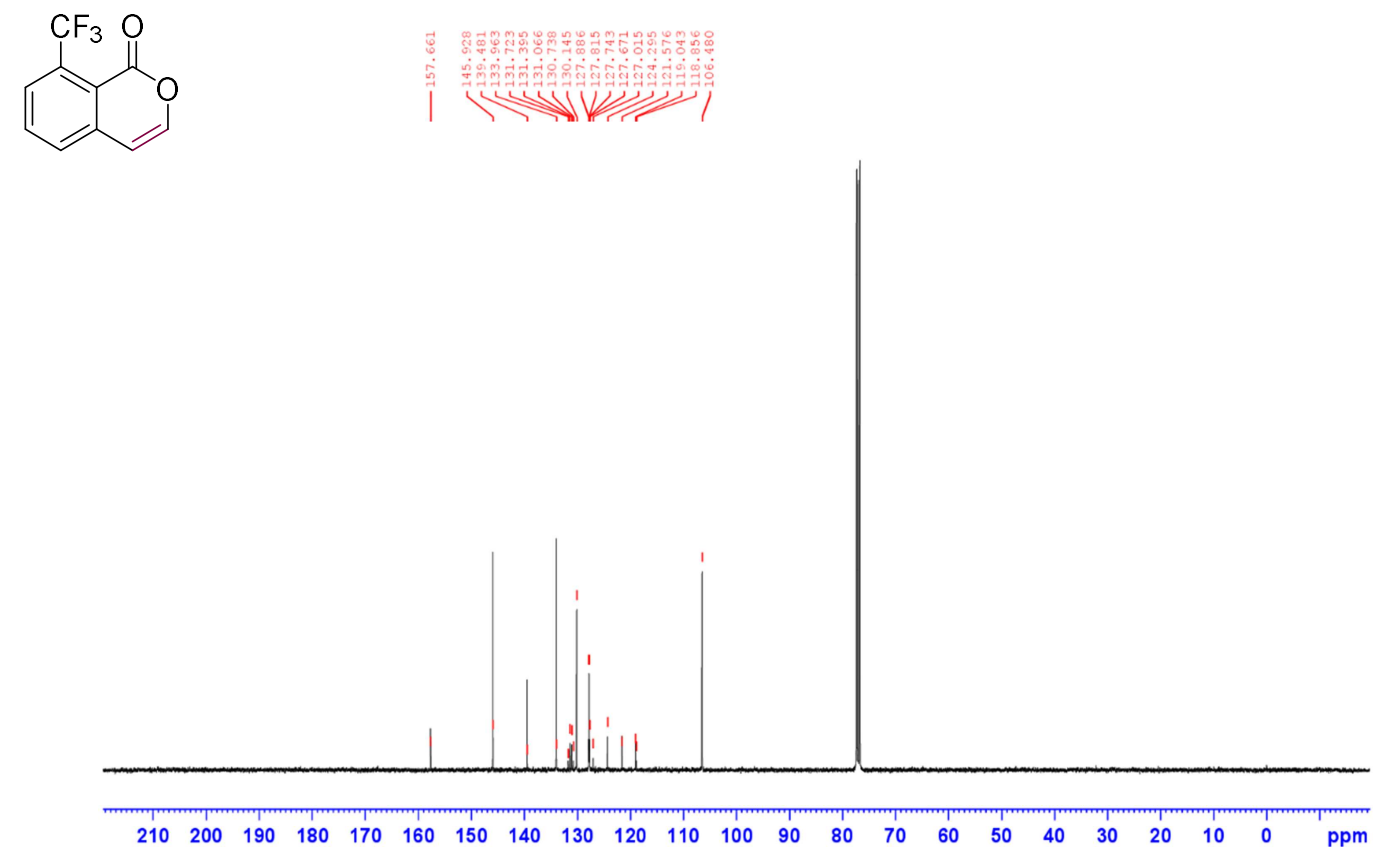


${ }^{19} \mathrm{~F}$ NMR of $30\left(400 \mathrm{MHz}, \mathrm{CDCl}_{3}\right)$

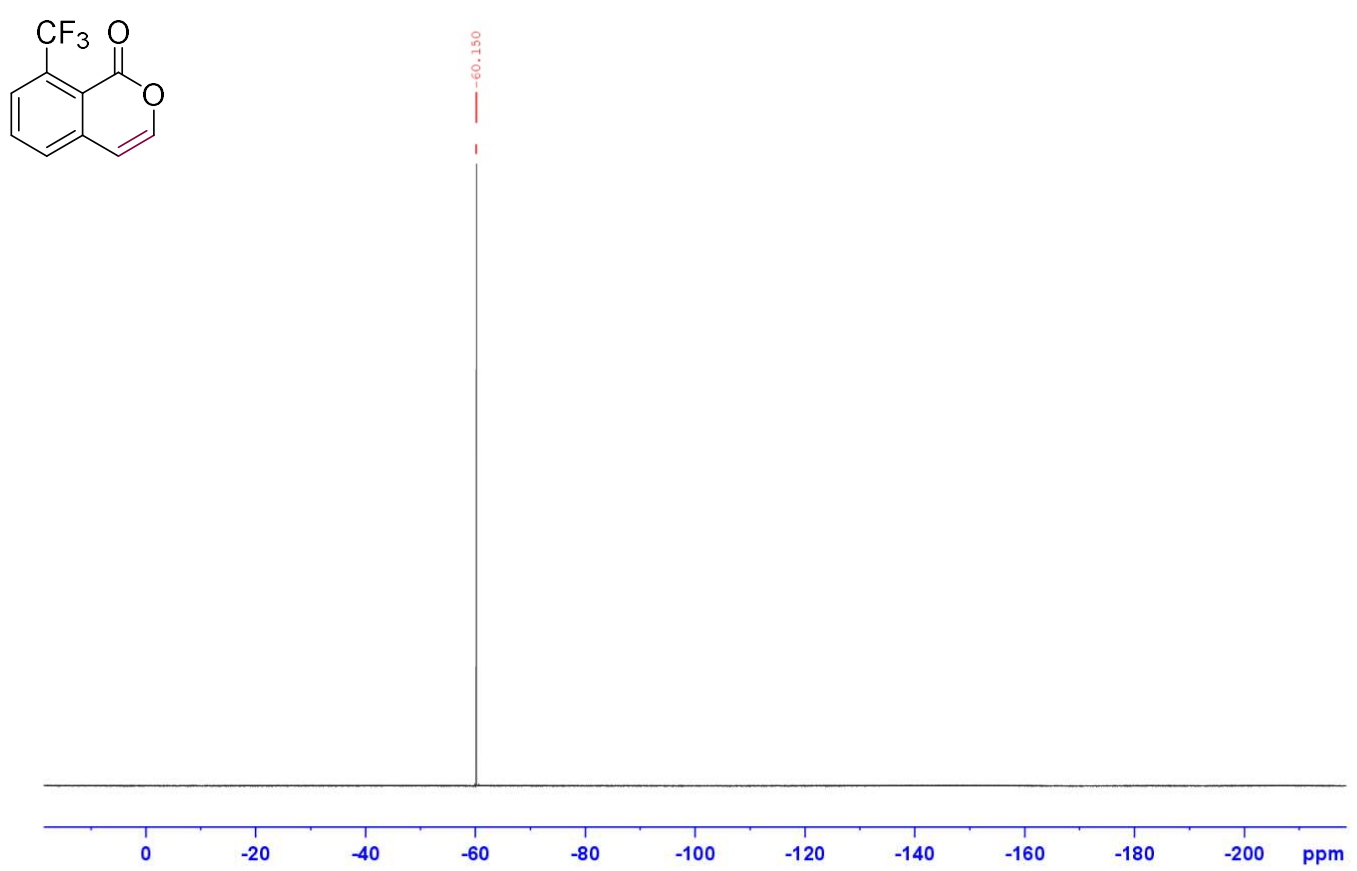


${ }^{1} \mathrm{H}$ NMR of $\mathbf{3 p}\left(400 \mathrm{MHz}, \mathrm{CDCl}_{3}\right)$<smiles>COc1cc2ccoc(=O)c2cc1OC</smiles>

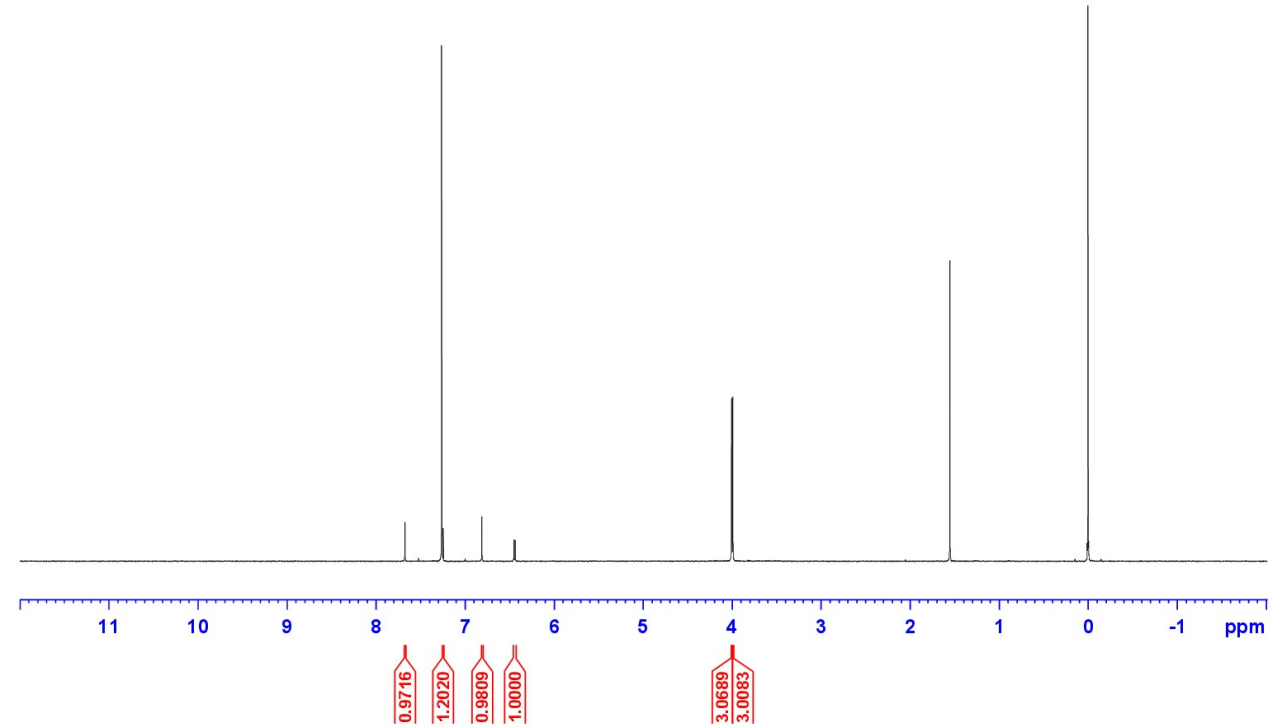

${ }^{13} \mathrm{C}$ NMR of $3 p\left(100 \mathrm{MHz}, \mathrm{CDCl}_{3}\right)$<smiles>COc1cc2ccoc(=O)c2cc1OC</smiles> 
${ }^{1} \mathrm{H} \mathrm{NMR}$ of $\mathbf{3 q}\left(400 \mathrm{MHz}, \mathrm{CDCl}_{3}\right)$

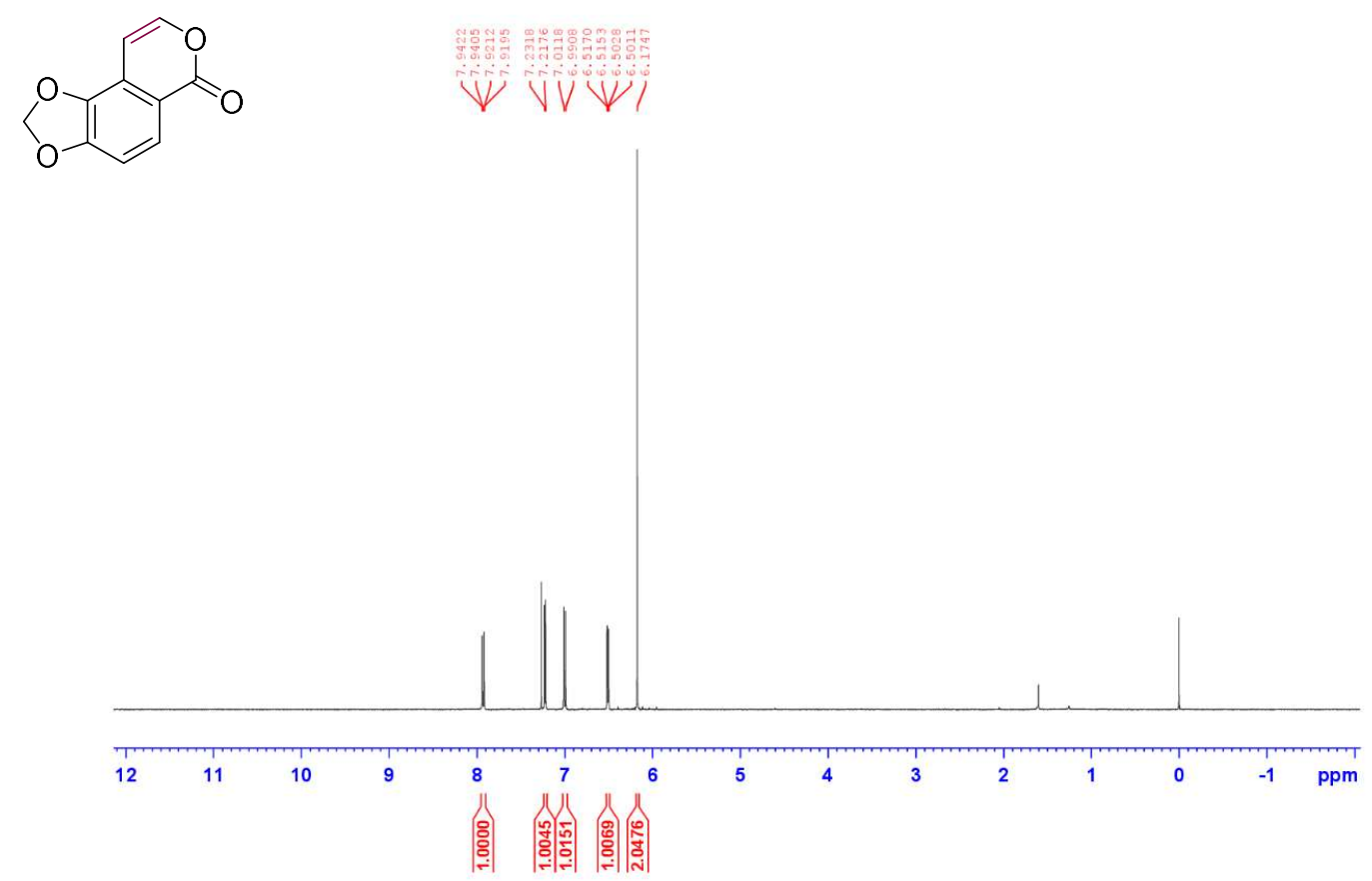

${ }^{13} \mathrm{C}$ NMR of $\mathbf{3 q}\left(100 \mathrm{MHz}, \mathrm{CDCl}_{3}\right)$
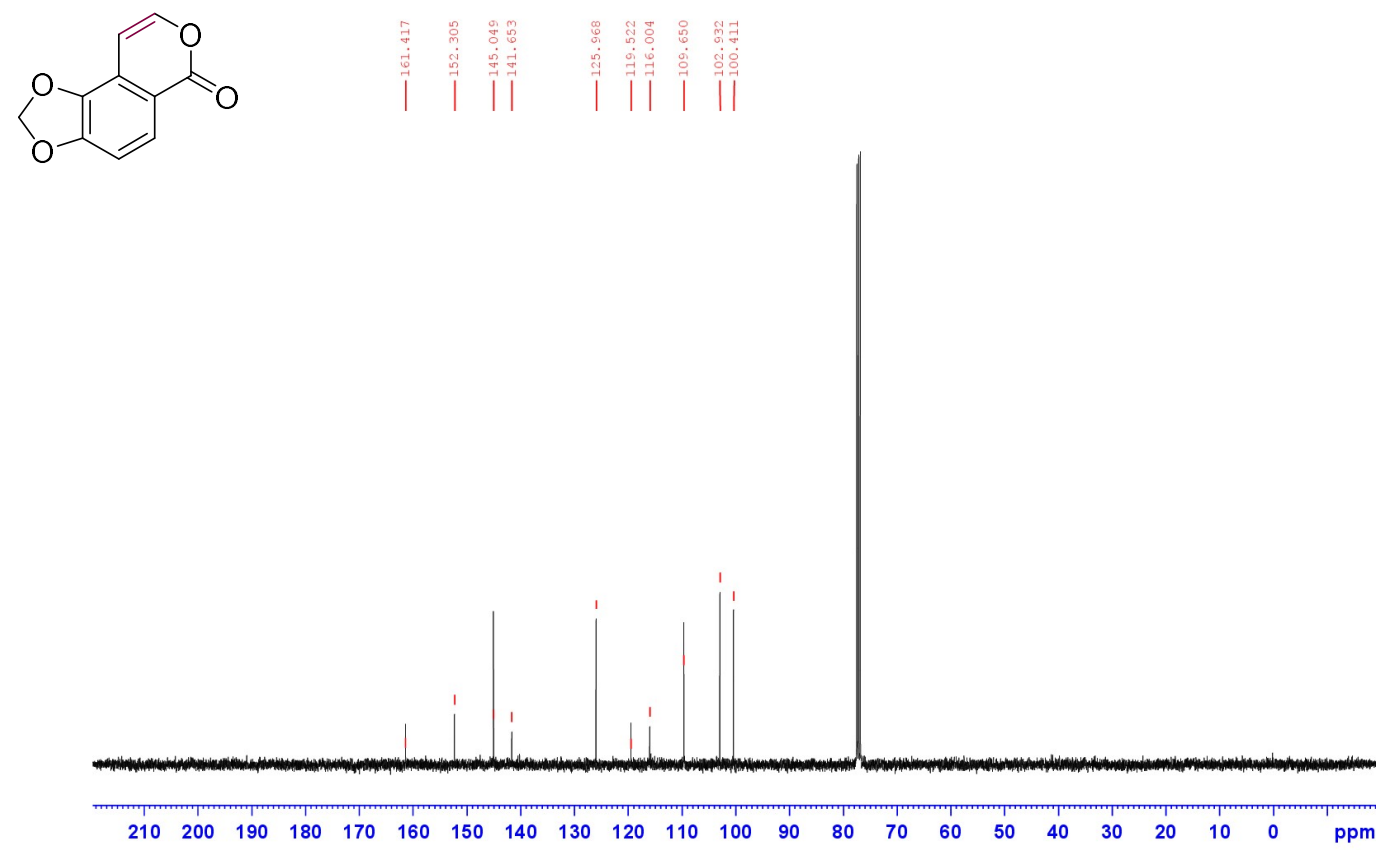
${ }^{1} \mathrm{H}$ NMR of $3 r\left(400 \mathrm{MHz}, \mathrm{CDCl}_{3}\right)$

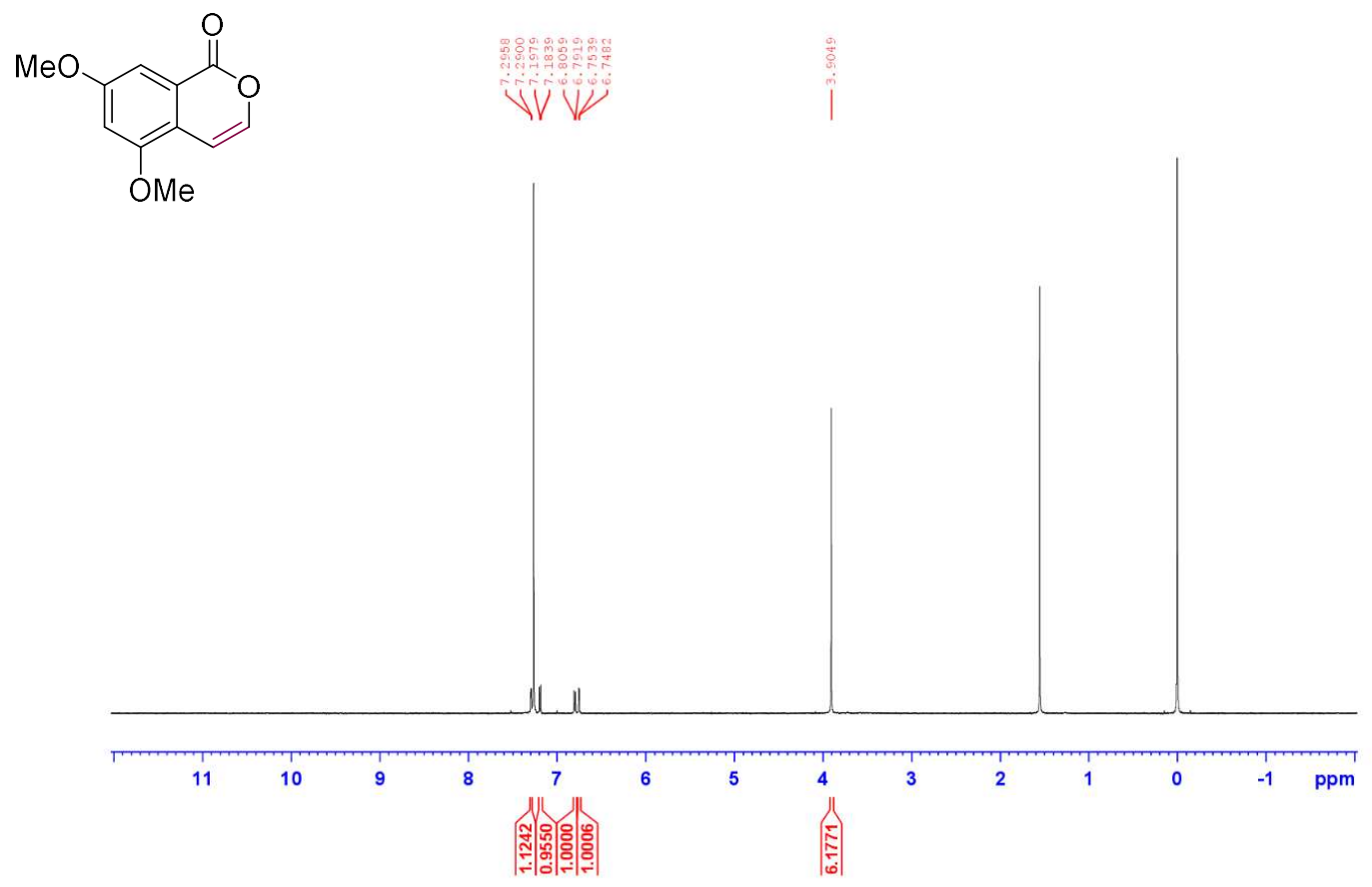

${ }^{13} \mathrm{C}$ NMR of $3 \mathbf{r}\left(100 \mathrm{MHz}, \mathrm{CDCl}_{3}\right)$<smiles></smiles>

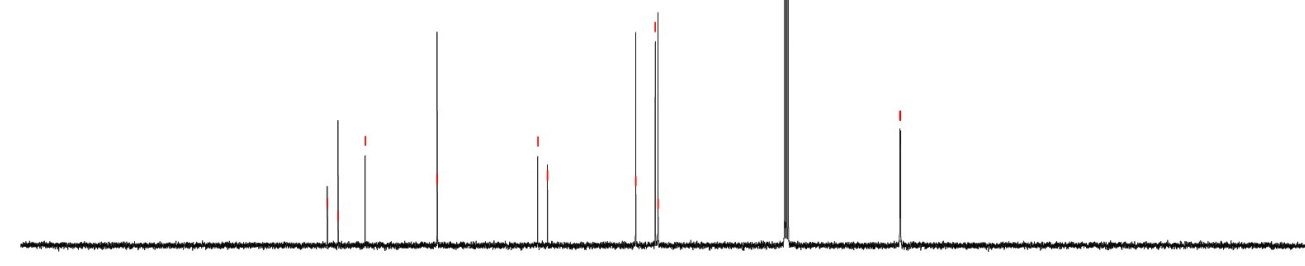

$\begin{array}{lllllllllllllllllllllll}210 & 200 & 190 & 180 & 170 & 160 & 150 & 140 & 130 & 120 & 110 & 100 & 90 & 80 & 70 & 60 & 50 & 40 & 30 & 20 & 10 & 0 & \mathrm{ppm}\end{array}$ 
${ }^{1} \mathrm{H}$ NMR of 3s $\left(400 \mathrm{MHz}, \mathrm{CDCl}_{3}\right)$

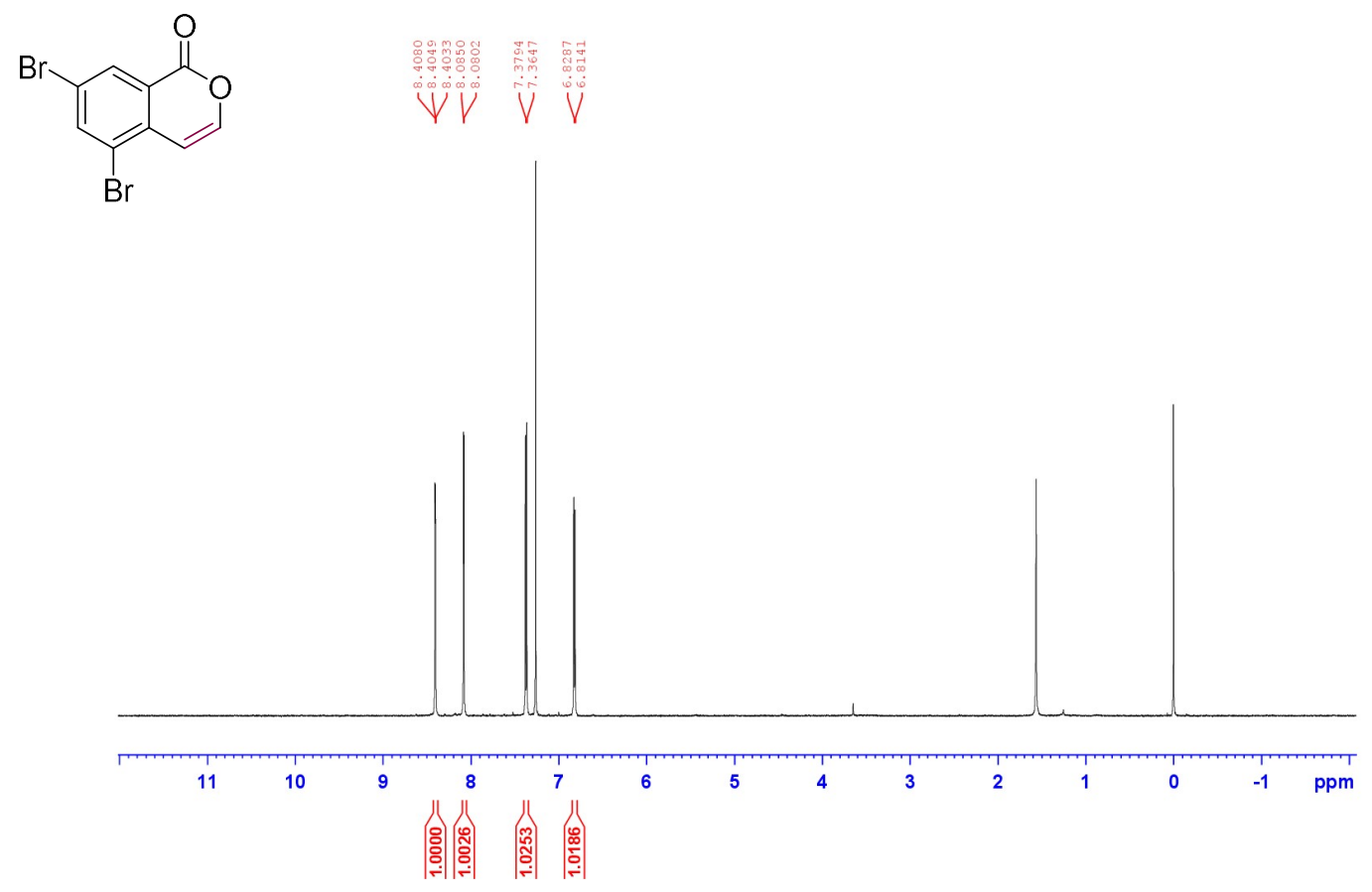

${ }^{13} \mathrm{C}$ NMR of $3 s\left(100 \mathrm{MHz}, \mathrm{CDCl}_{3}\right)$

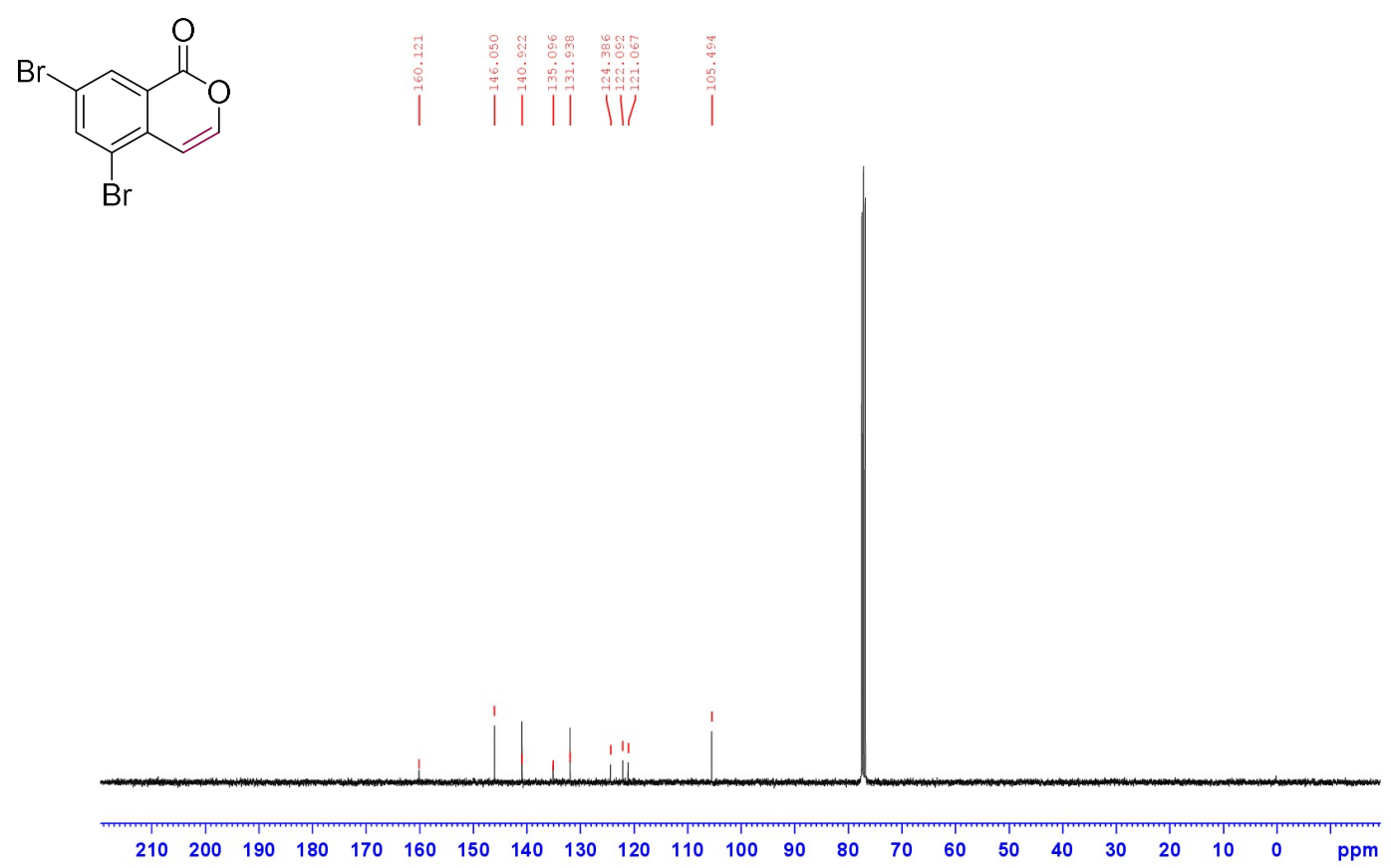


${ }^{1} \mathrm{H}$ NMR of $3 t\left(400 \mathrm{MHz}, \mathrm{CDCl}_{3}\right)$

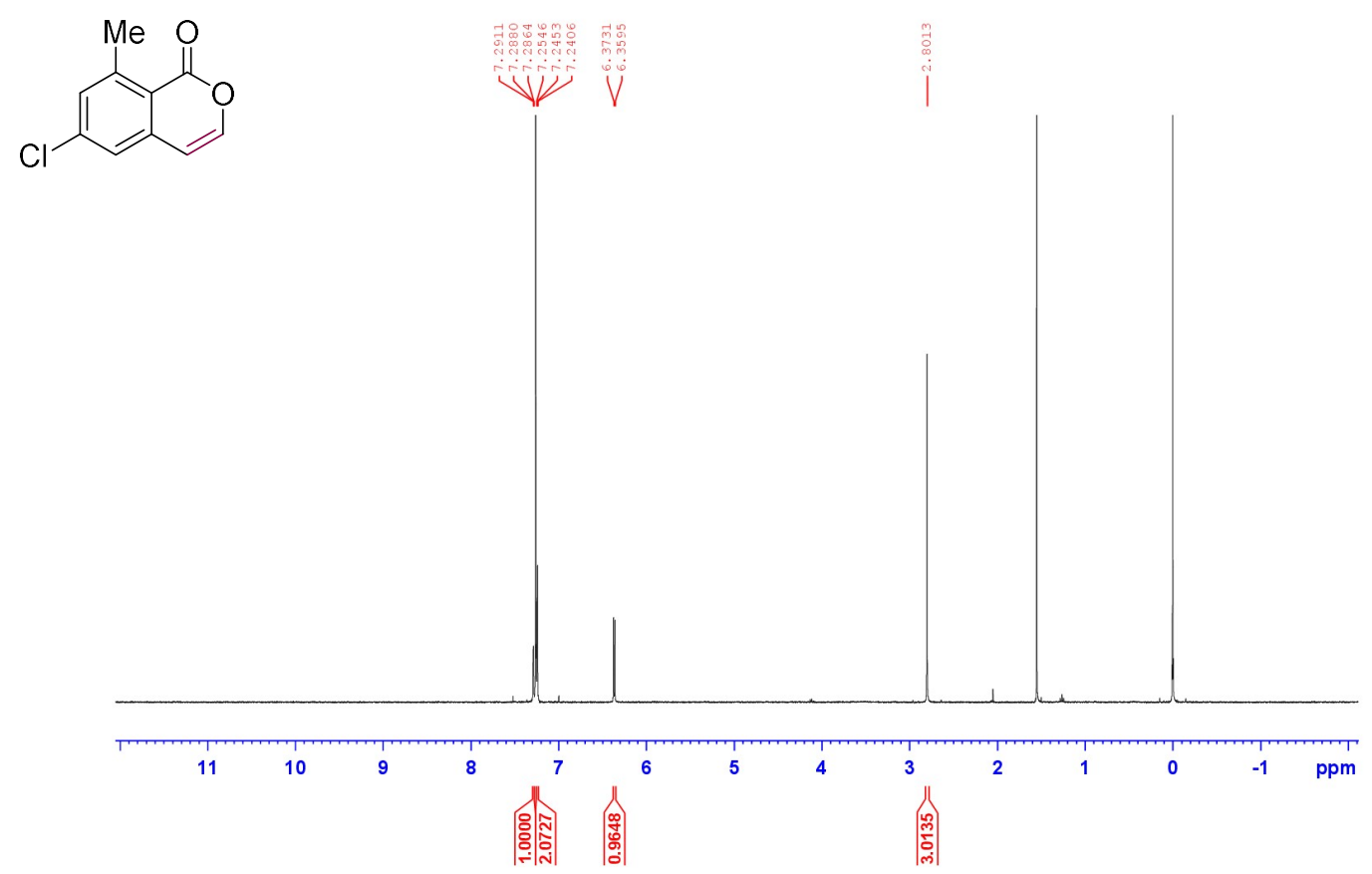

${ }^{13} \mathrm{C}$ NMR of $3 t\left(100 \mathrm{MHz}, \mathrm{CDCl}_{3}\right)$<smiles>Cc1cc(Cl)cc2ccoc(=O)c12</smiles>

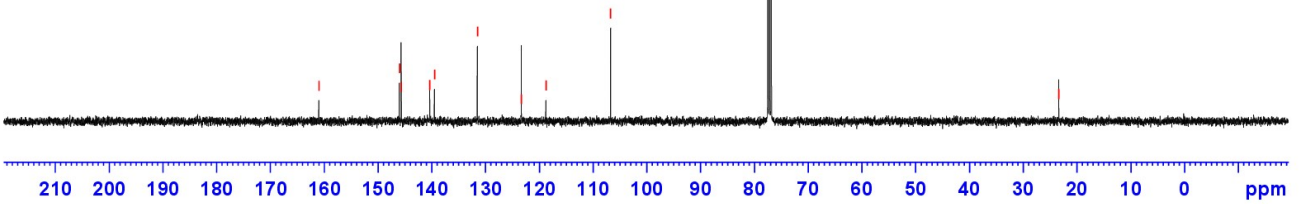


${ }^{1} \mathrm{H}$ NMR of $\mathbf{3 u}\left(400 \mathrm{MHz}, \mathrm{CDCl}_{3}\right)$
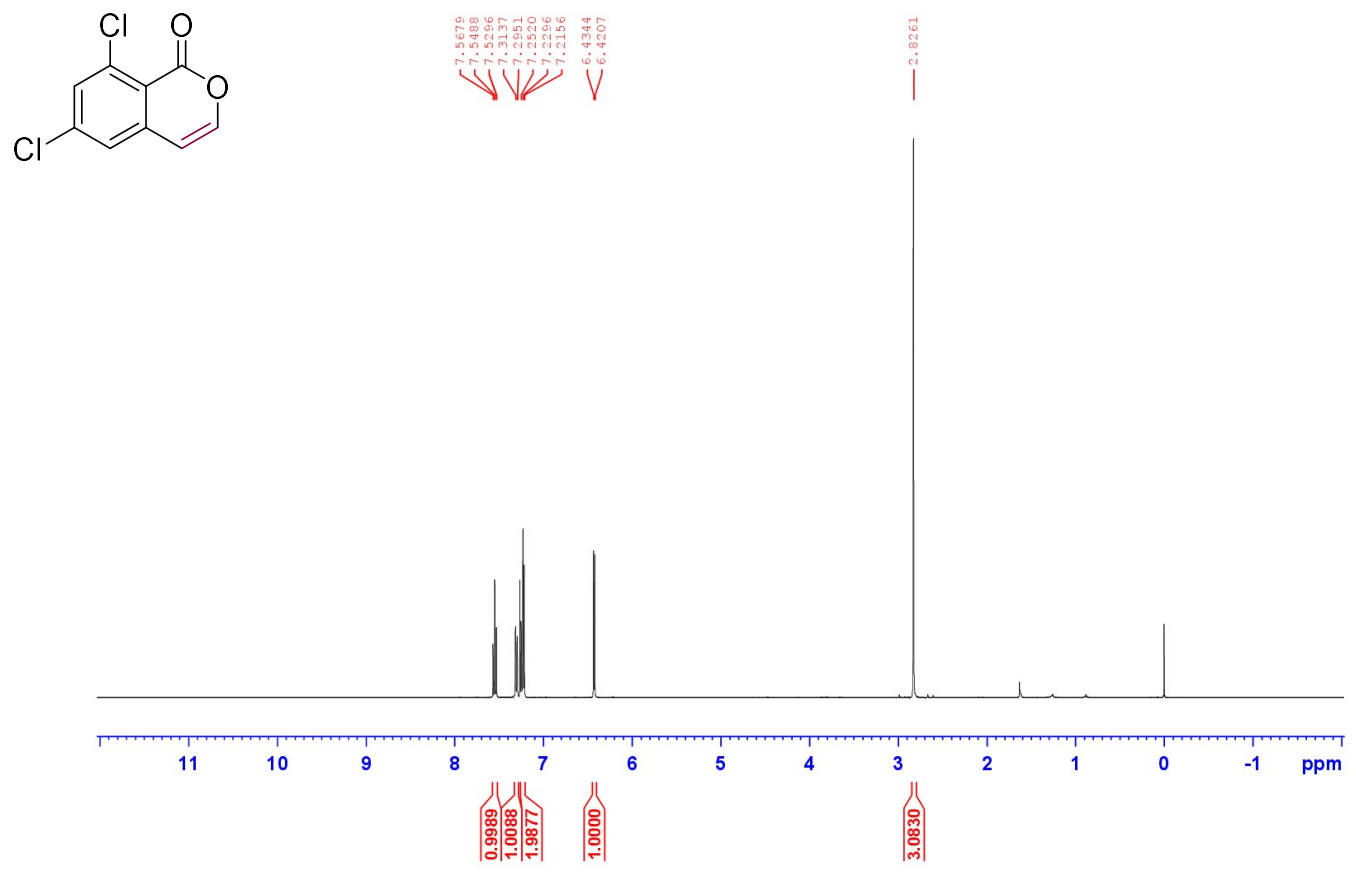

${ }^{13} \mathrm{C}$ NMR of $3 \mathbf{u}\left(100 \mathrm{MHz}, \mathrm{CDCl}_{3}\right)$
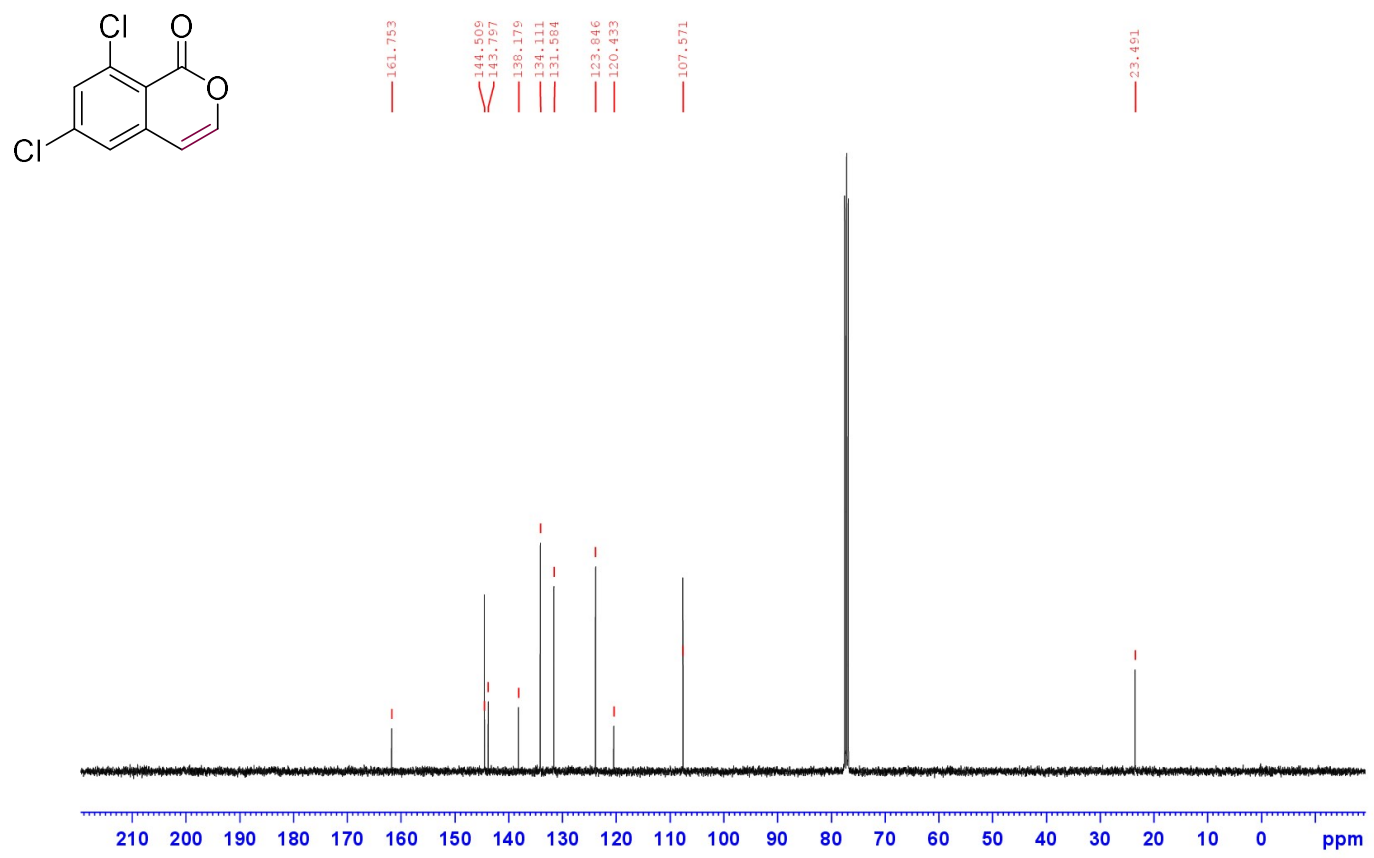

S43 
${ }^{1} \mathrm{H} \mathrm{NMR}$ of $\mathbf{3 v}\left(400 \mathrm{MHz}, \mathrm{CDCl}_{3}\right)$<smiles>O=c1occc2cc3ccccc3cc12</smiles>
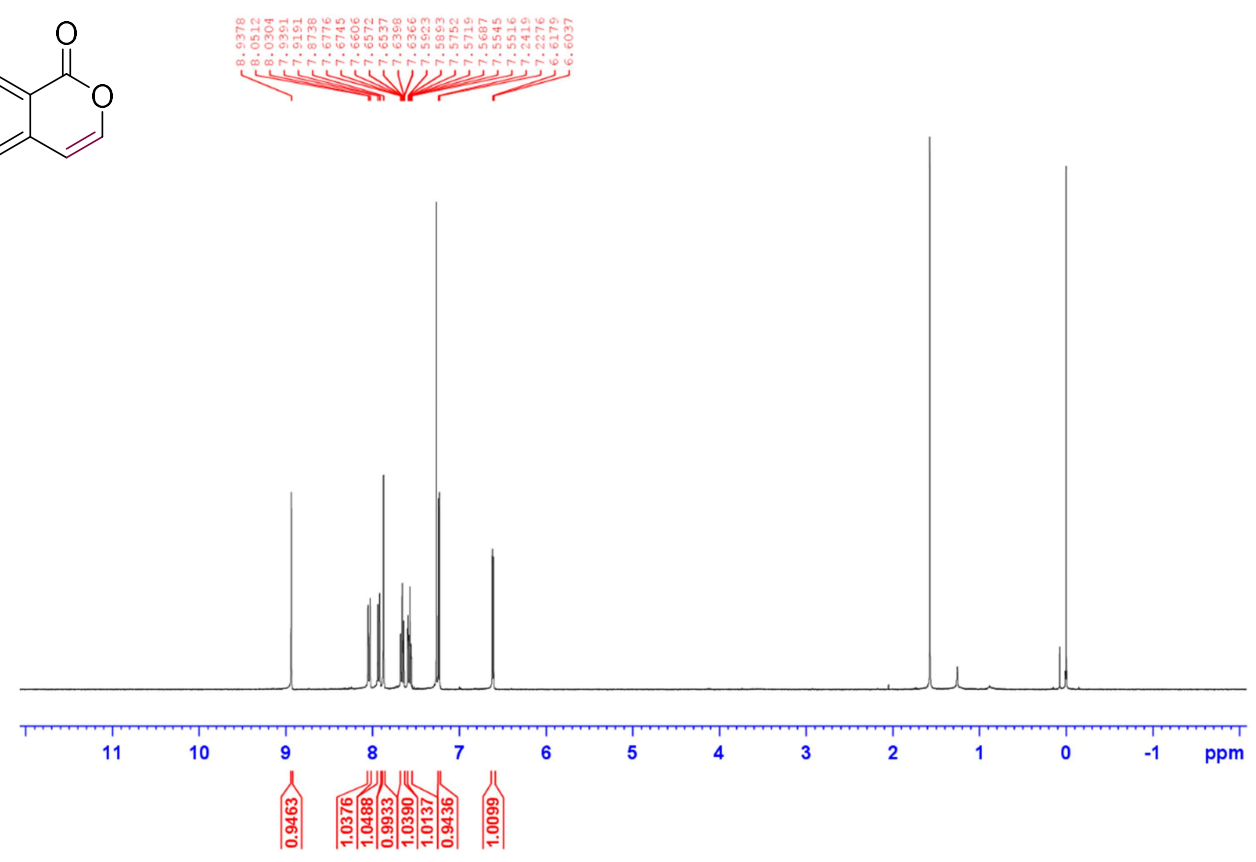

${ }^{13} \mathrm{C}$ NMR of $3 \mathbf{v}\left(100 \mathrm{MHz}, \mathrm{CDCl}_{3}\right)$<smiles>O=c1occc2cc3ccccc3cc12</smiles>

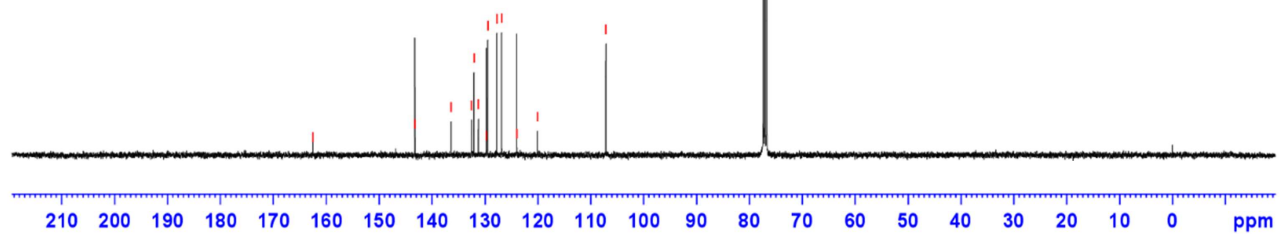


${ }^{1} \mathrm{H}$ NMR of 3w $\left(400 \mathrm{MHz}, \mathrm{CDCl}_{3}\right)$

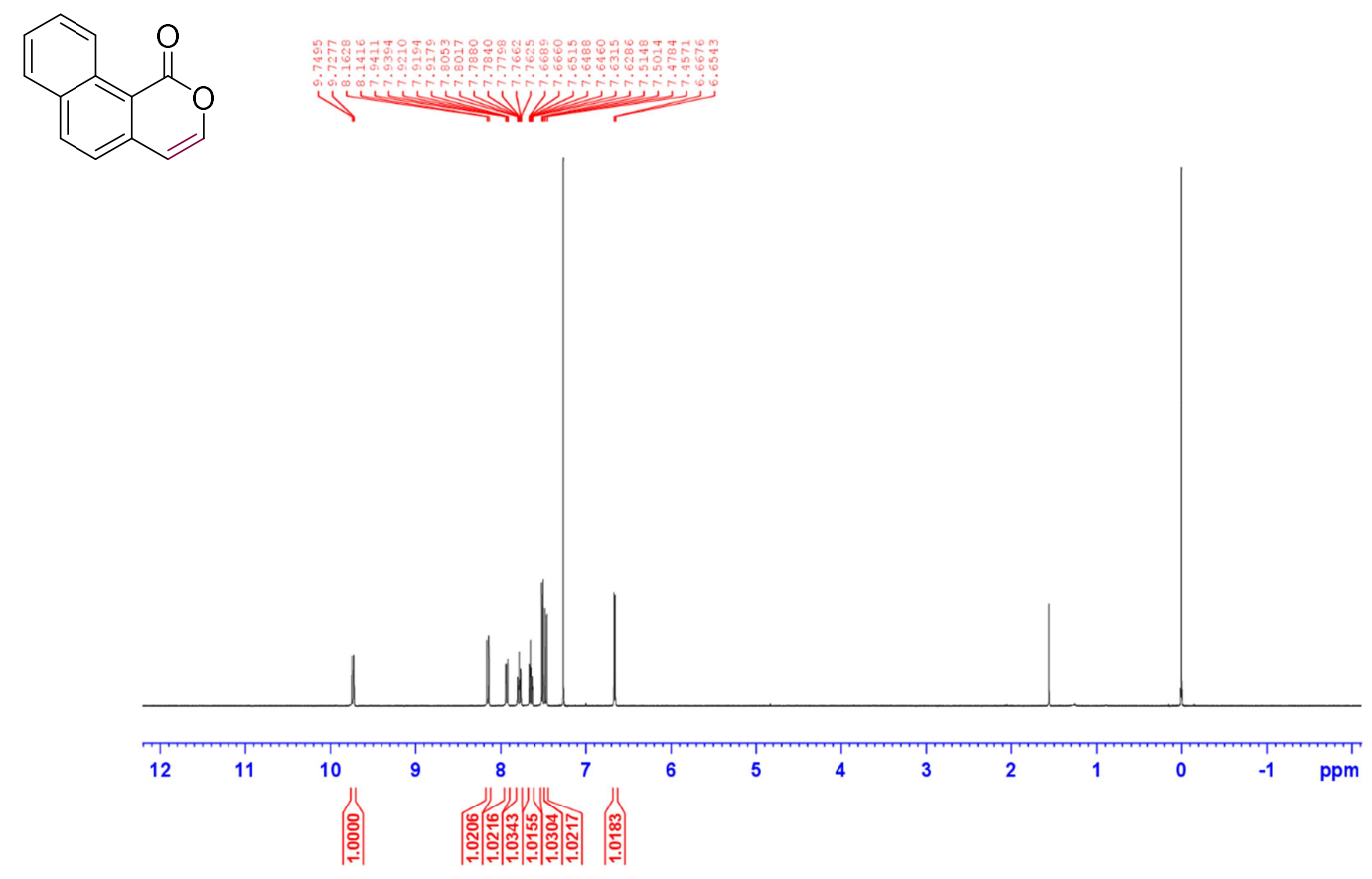

${ }^{13} \mathrm{C}$ NMR of $\mathbf{3 w}\left(100 \mathrm{MHz}, \mathrm{CDCl}_{3}\right)$<smiles>O=c1occc2ccc3ccccc3c12</smiles>

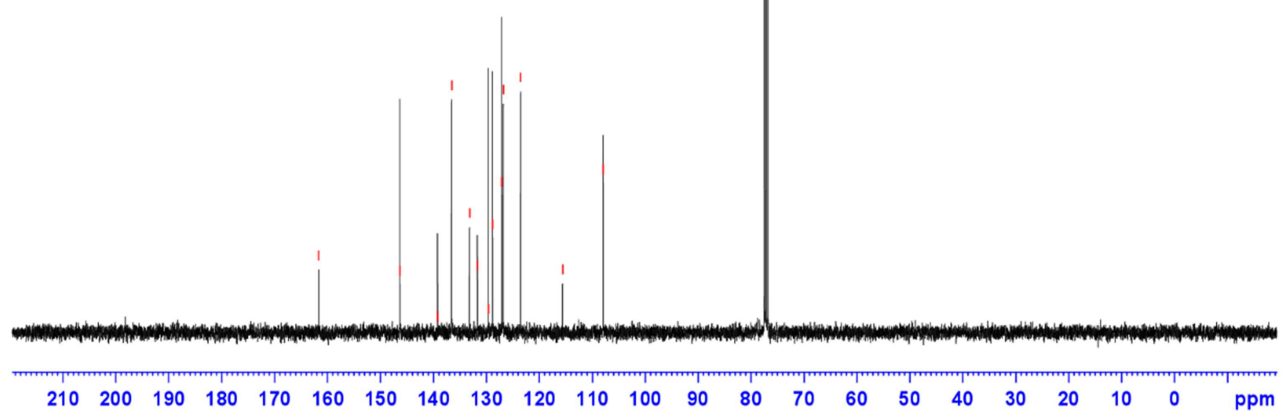


${ }^{1} \mathrm{H}$ NMR of 3x $\left(400 \mathrm{MHz}, \mathrm{CDCl}_{3}\right)$

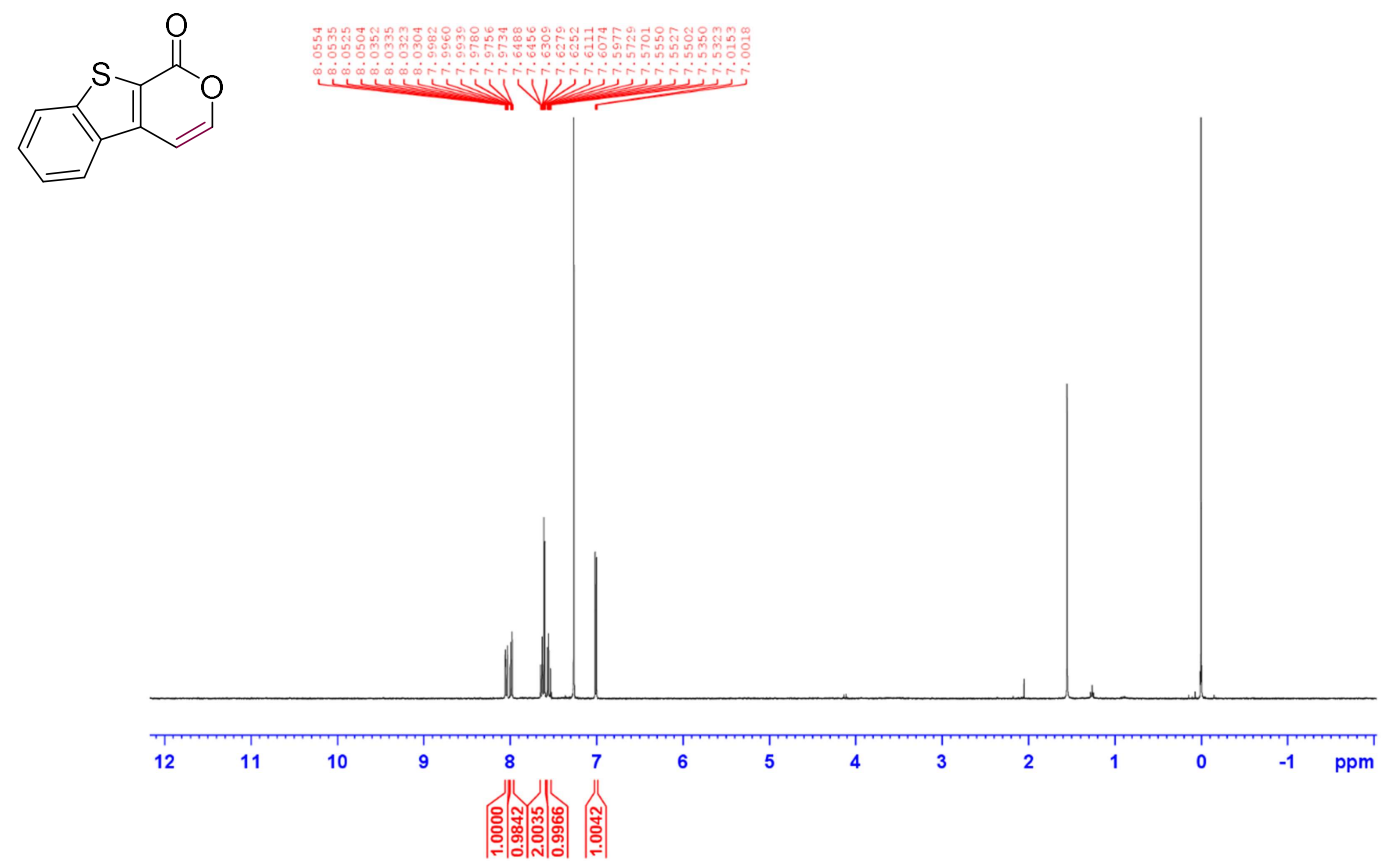

${ }^{13} \mathrm{C}$ NMR of $3 \mathbf{x}\left(100 \mathrm{MHz}, \mathrm{CDCl}_{3}\right)$<smiles>O=c1occc2c1sc1ccccc12</smiles>

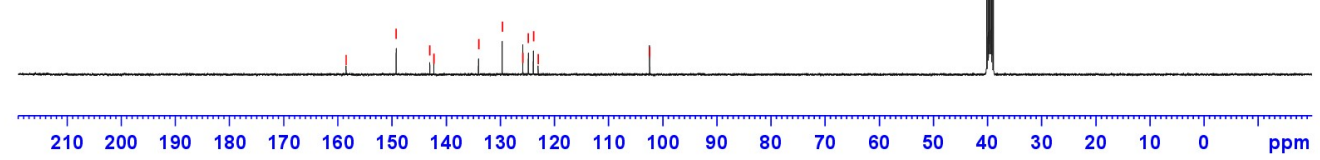


${ }^{1} \mathrm{H}$ NMR of $\mathbf{3 y}\left(400 \mathrm{MHz}, \mathrm{CDCl}_{3}\right)$

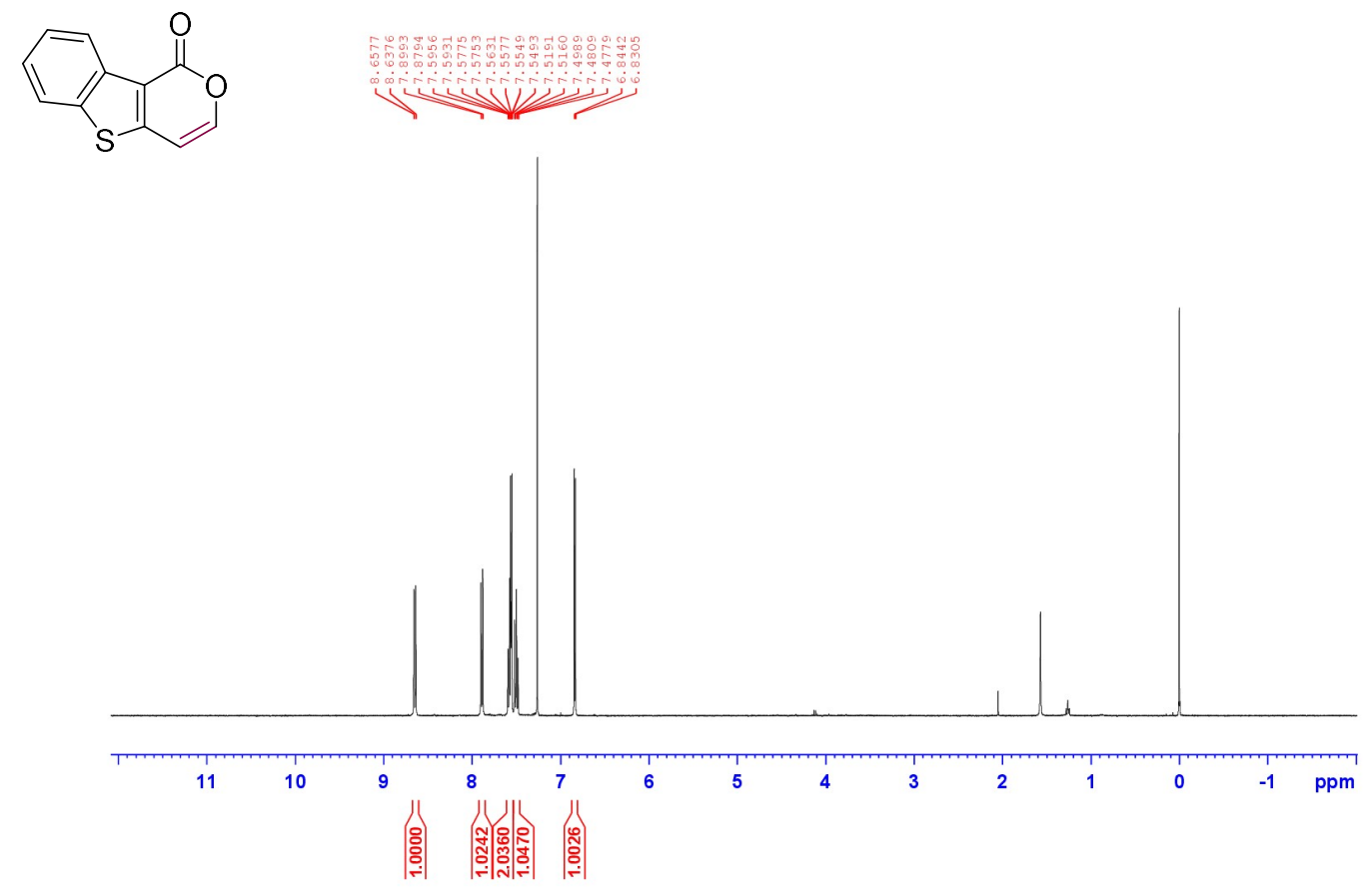

${ }^{13} \mathrm{C}$ NMR of $\mathbf{3 y}\left(100 \mathrm{MHz}, \mathrm{CDCl}_{3}\right)$
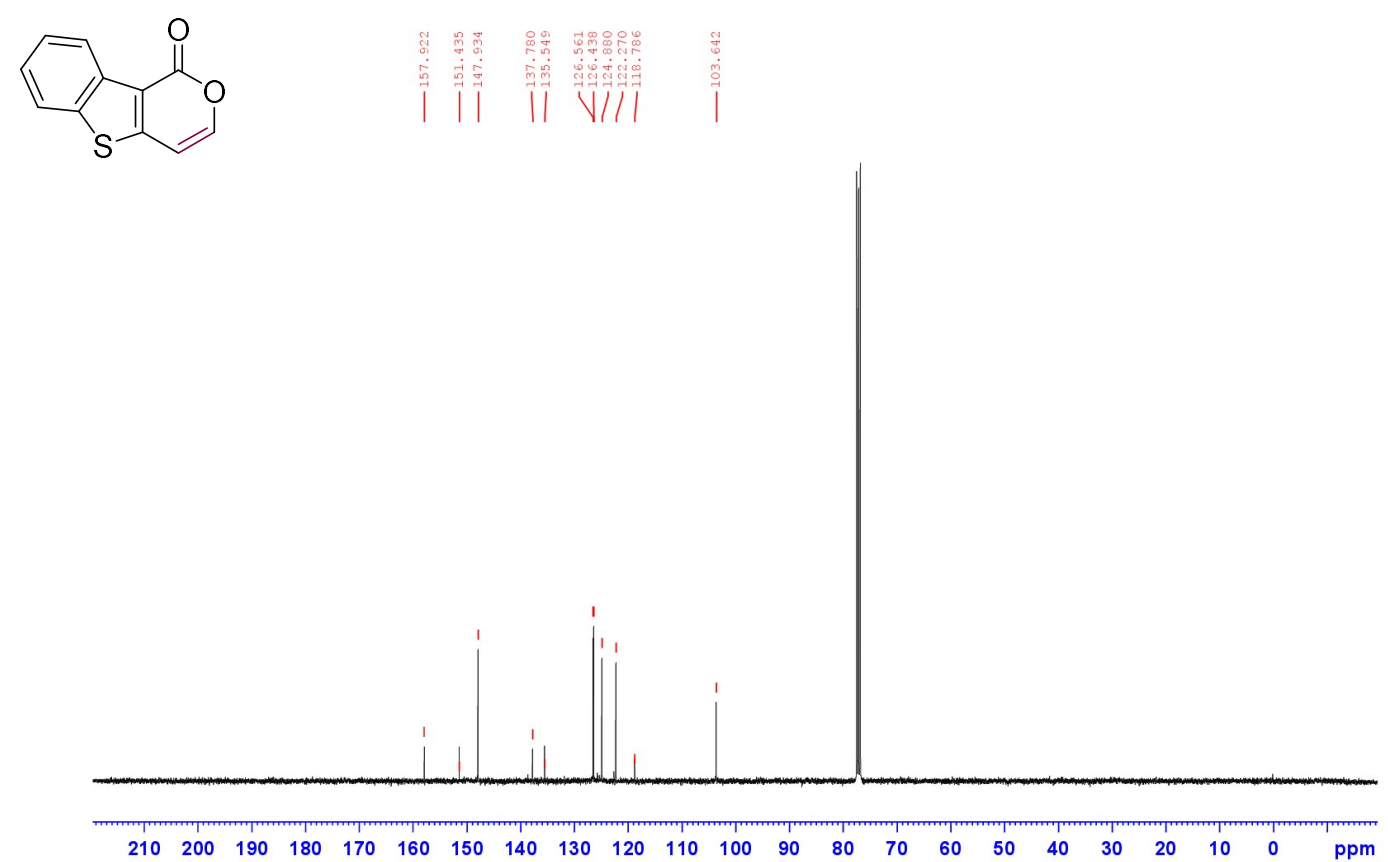
${ }^{1} \mathrm{H}$ NMR of $4\left(400 \mathrm{MHz}, \mathrm{CDCl}_{3}\right)$

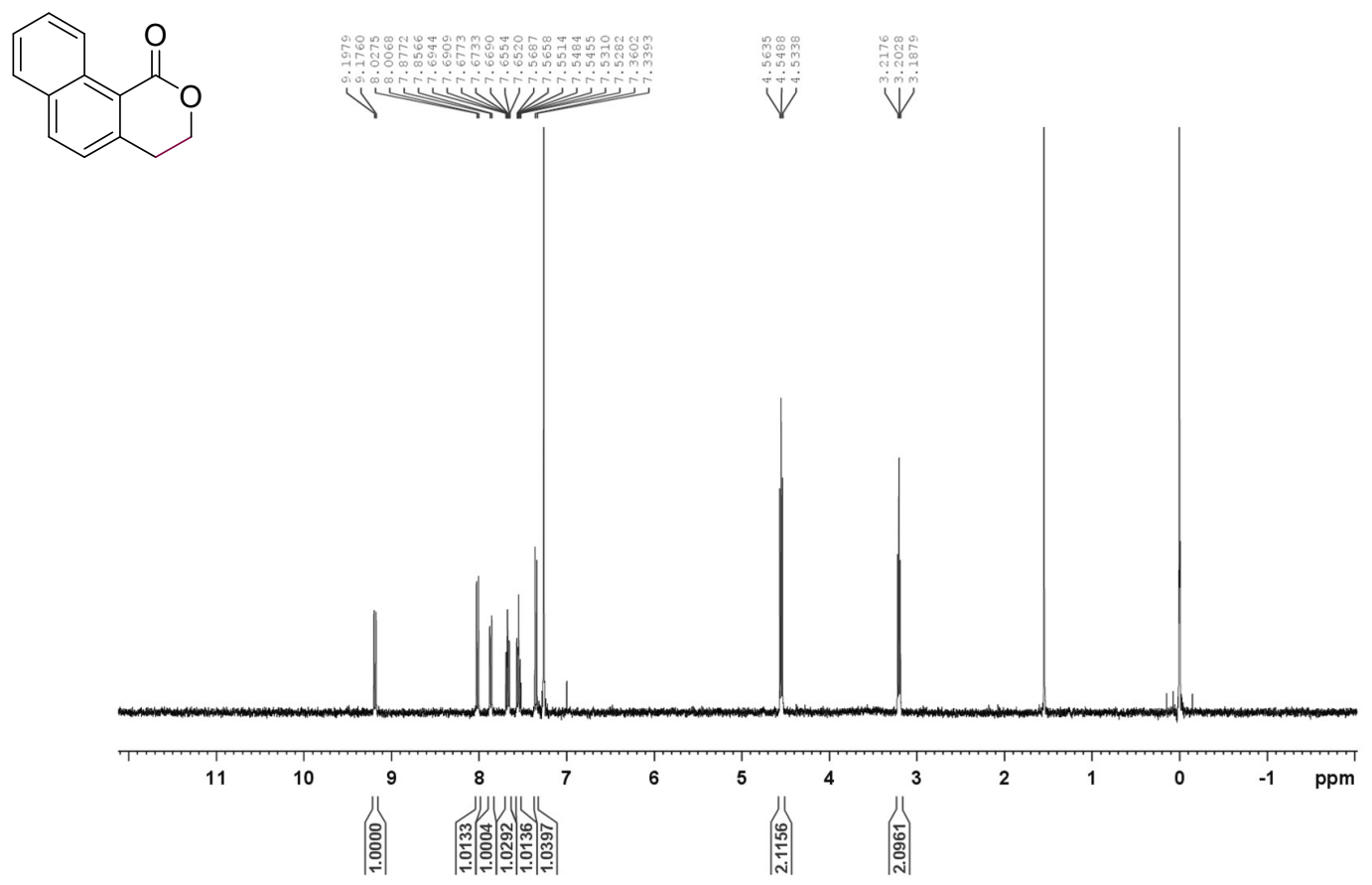

${ }^{13} \mathrm{C}$ NMR of $4\left(100 \mathrm{MHz}, \mathrm{CDCl}_{3}\right)$<smiles>O=C1OCCc2ccc3ccccc3c21</smiles>

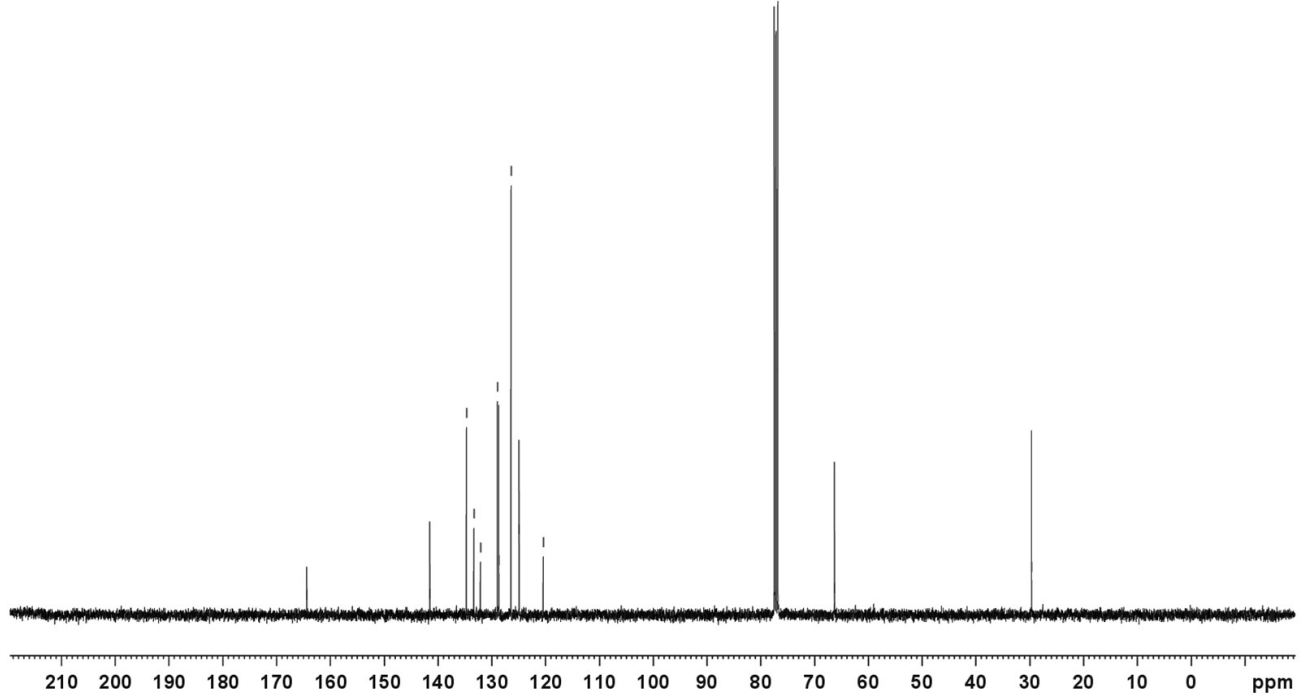


${ }^{1} \mathrm{H}$ NMR of $5\left(400 \mathrm{MHz}, \mathrm{CDCl}_{3}\right)$
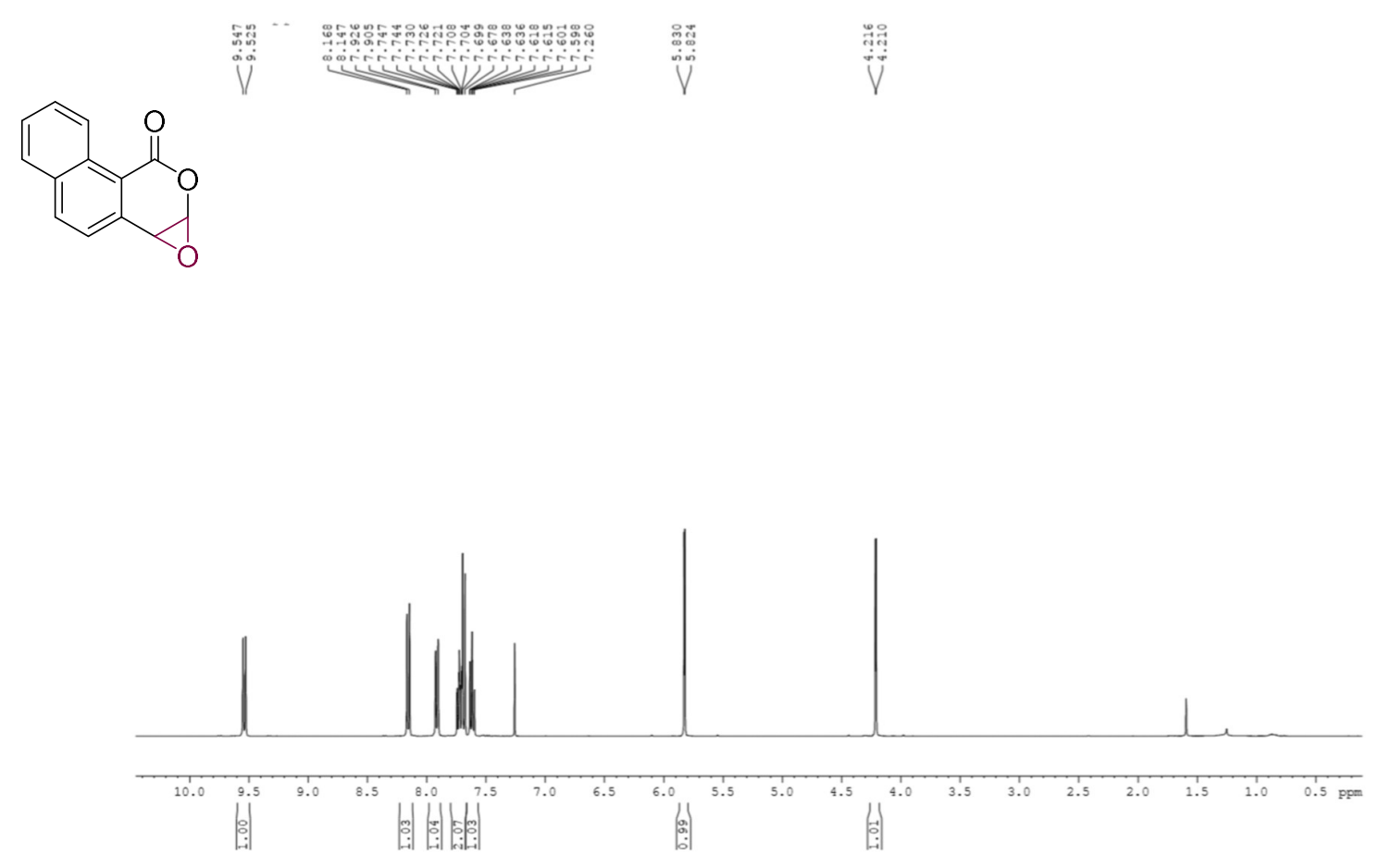

${ }^{13} \mathrm{C} \mathrm{NMR}$ of $5\left(100 \mathrm{MHz}, \mathrm{CDCl}_{3}\right)$
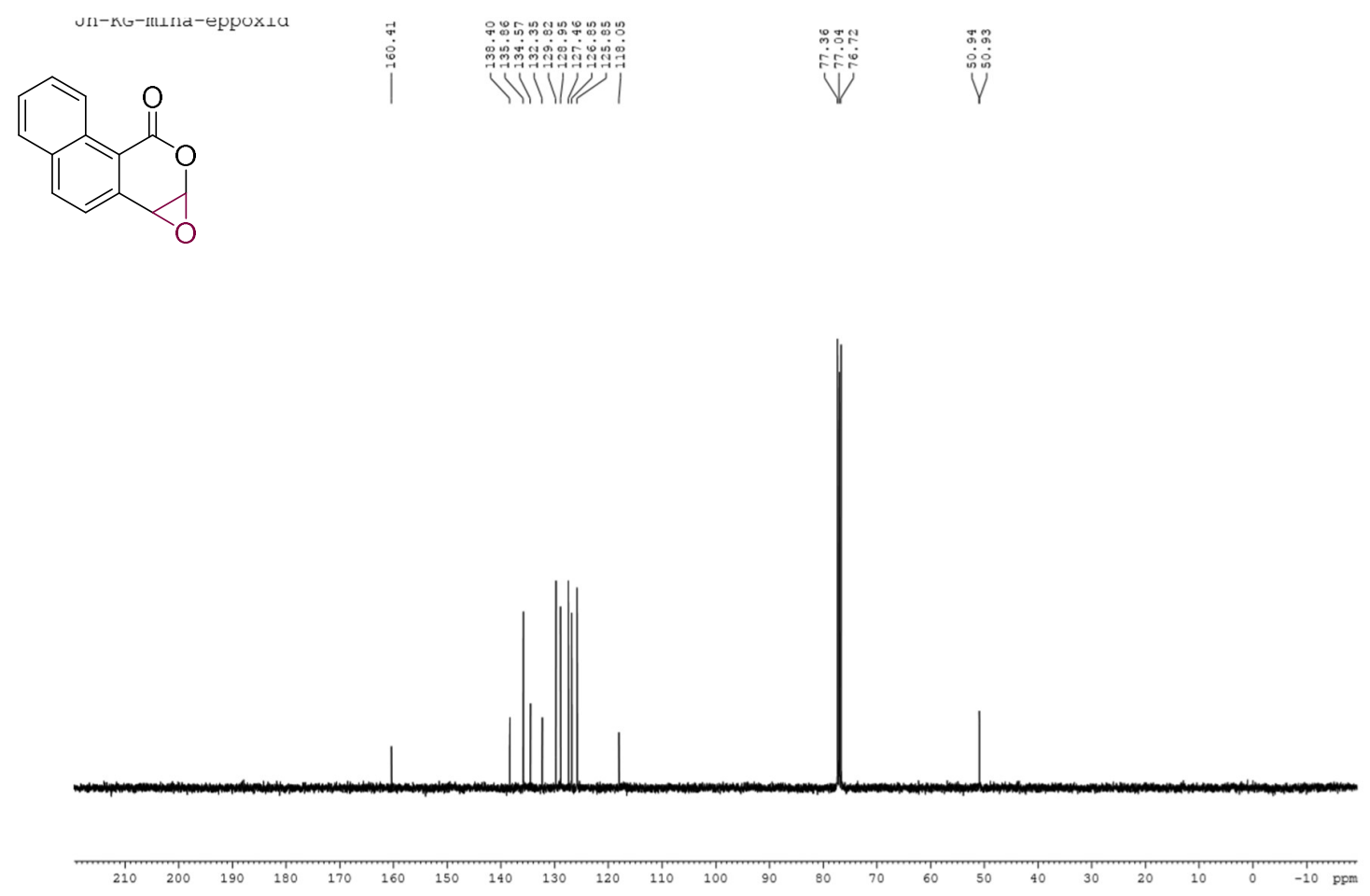
${ }^{1} \mathrm{H}$ NMR of $6\left(400 \mathrm{MHz}, \mathrm{CDCl}_{3}\right.$ )<smiles>O=C1OC(Br)C(Br)c2ccc3ccccc3c21</smiles><smiles>[Al]C(=[W])C=[V]</smiles>

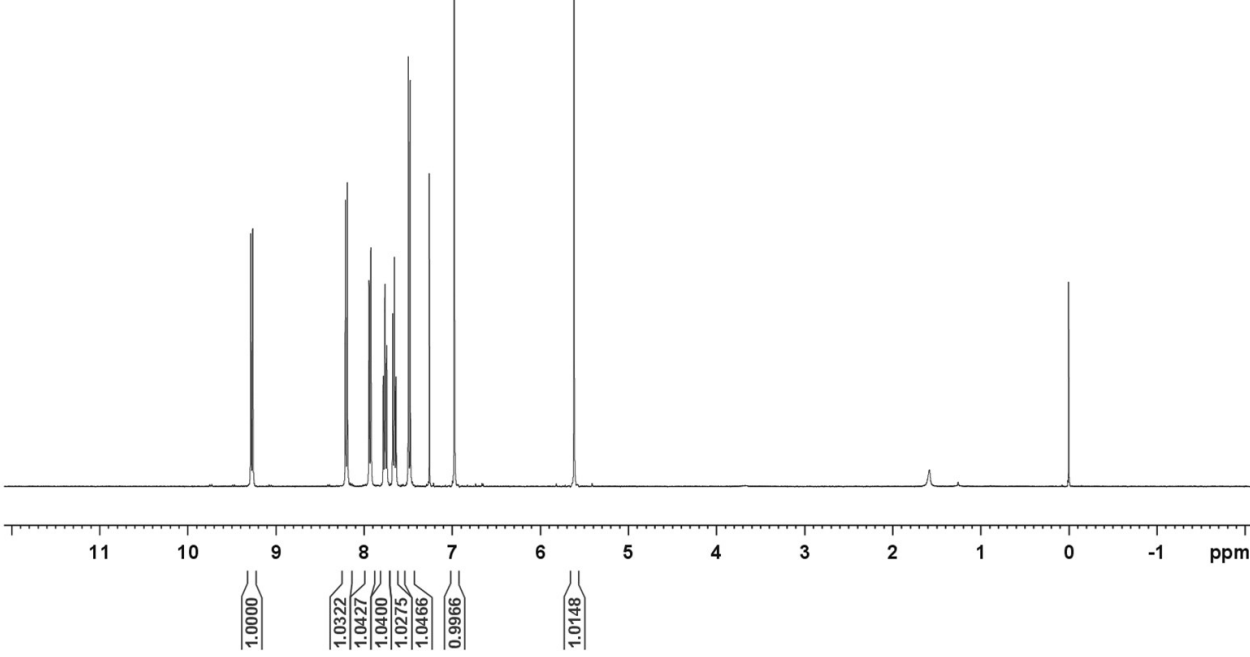

${ }^{13} \mathrm{C}$ NMR of $6\left(100 \mathrm{MHz}, \mathrm{CDCl}_{3}\right)$<smiles>O=C1OC(Br)C(Br)c2ccc3ccccc3c21</smiles>

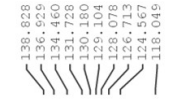

$\mathrm{Br}$

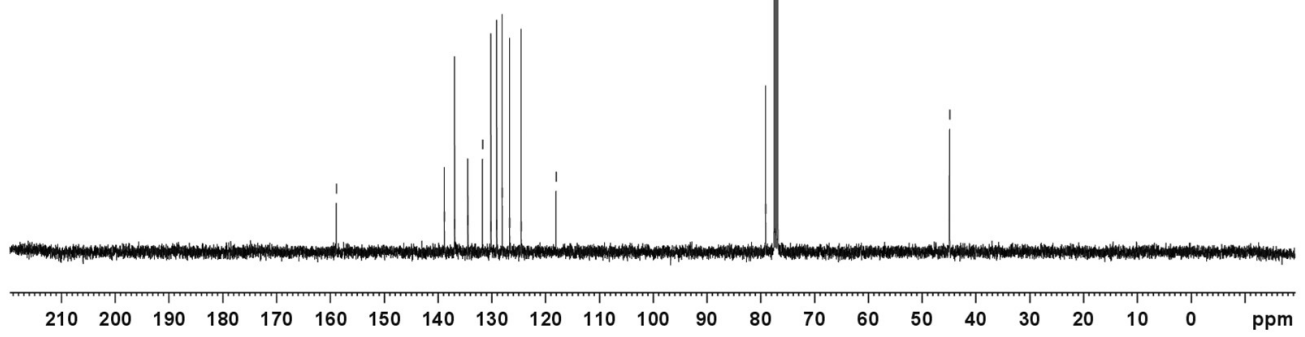


${ }^{1} \mathrm{H}$ NMR of 7 (400 MHz, $\mathrm{CDCl}_{3}$ )

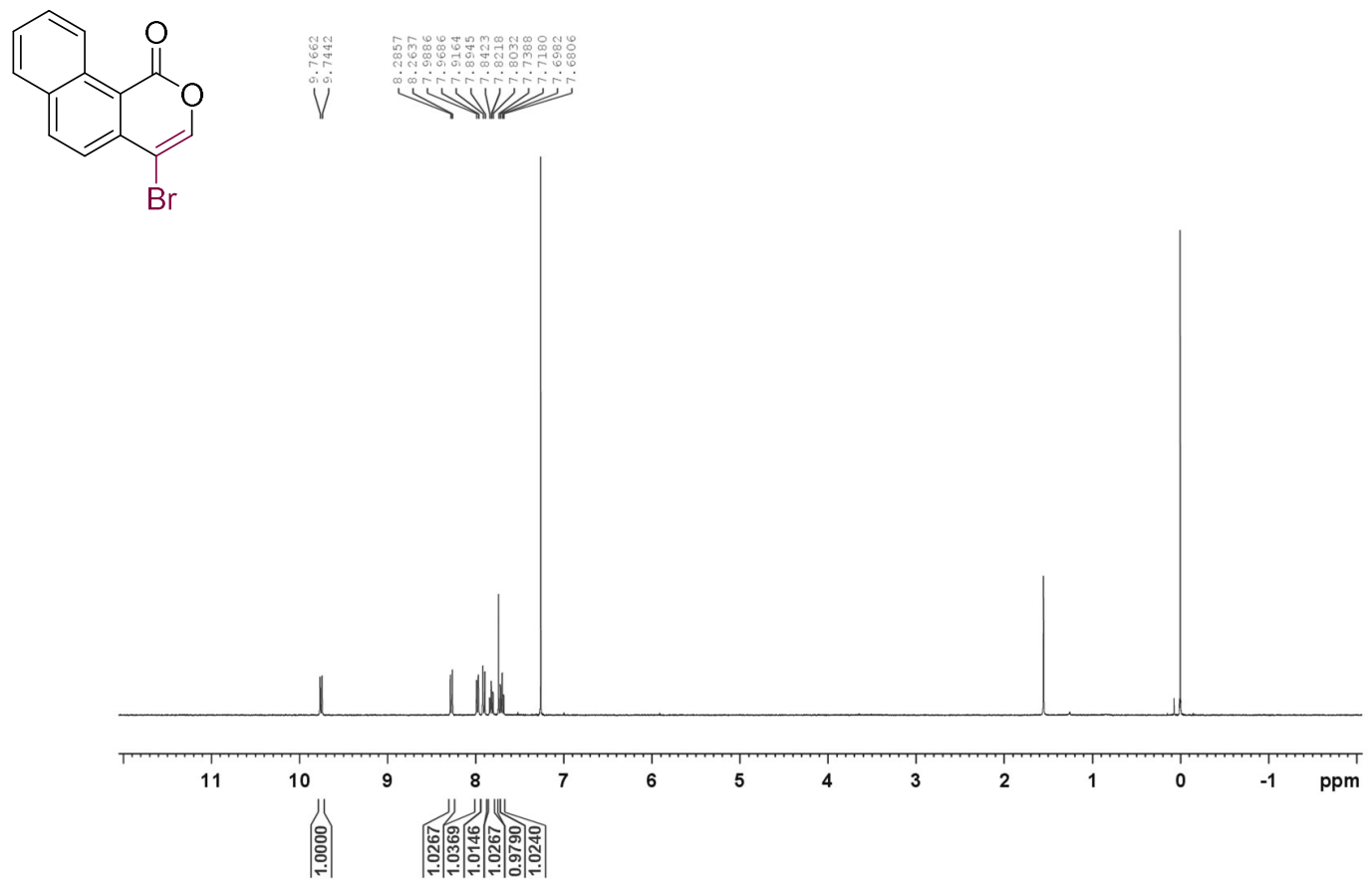

${ }^{13} \mathrm{C}$ NMR of $7\left(100 \mathrm{MHz}, \mathrm{CDCl}_{3}\right)$<smiles>O=c1occ(Br)c2ccc3ccccc3c12</smiles>

$\mathrm{Br}$

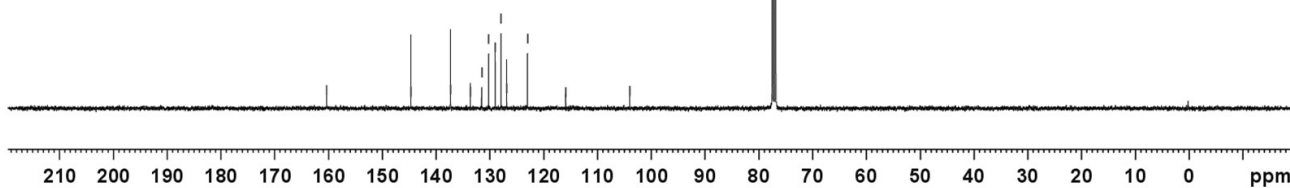


${ }^{1} \mathrm{H}$ NMR of $8\left(400 \mathrm{MHz}, \mathrm{CDCl}_{3}\right)$

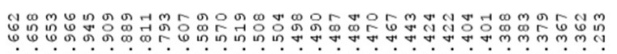

$\sqrt[0]{0}$

Vִ

$\underbrace{P h}_{0}$

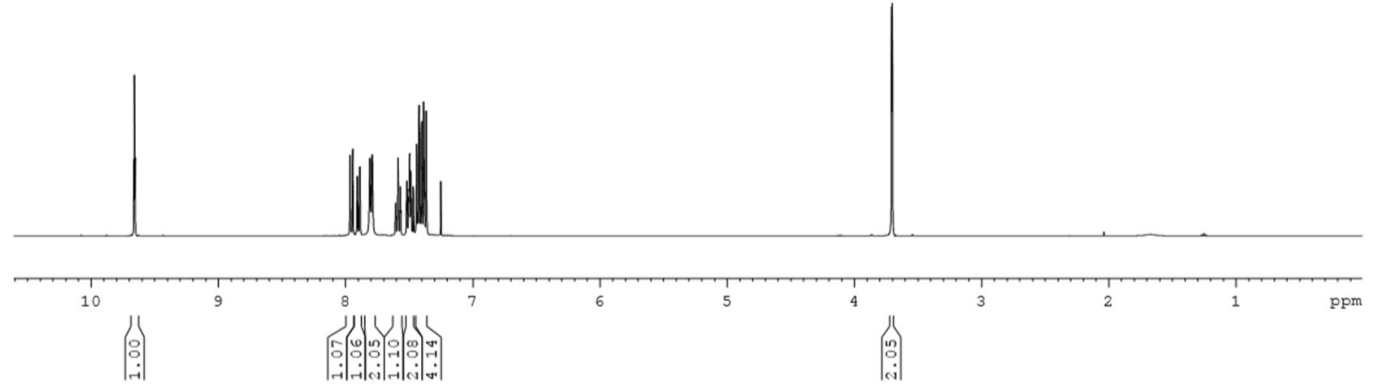

${ }^{13} \mathrm{C} \mathrm{NMR}$ of $8\left(100 \mathrm{MHz}, \mathrm{CDCl}_{3}\right)$

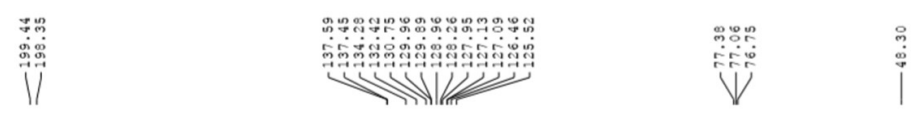

$\underbrace{2}$
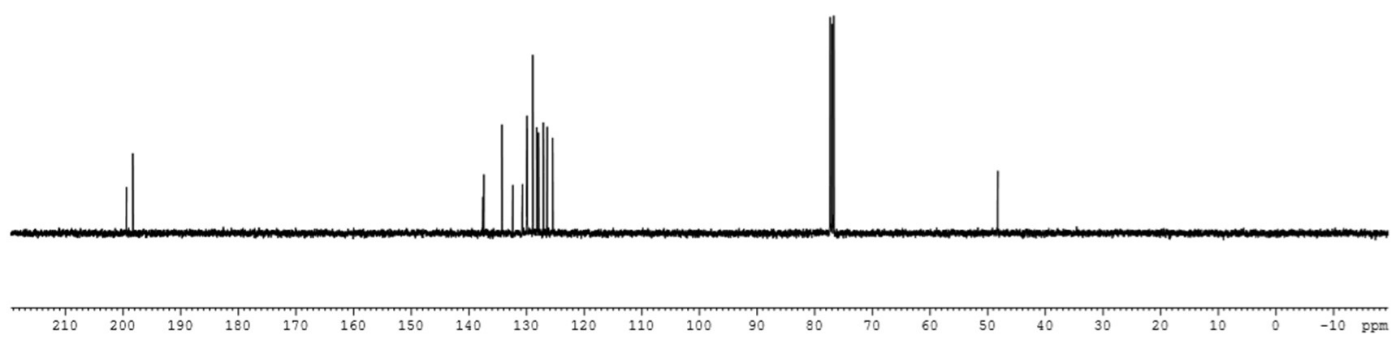
${ }^{1} \mathrm{H}$ NMR of $9\left(400 \mathrm{MHz}, \mathrm{CDCl}_{3}\right)$

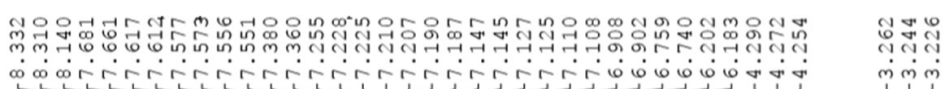

$\underbrace{\infty}$<smiles>O=c1c2ccc(Br)cc2ccn1CCc1c[nH]c2ccccc12</smiles>

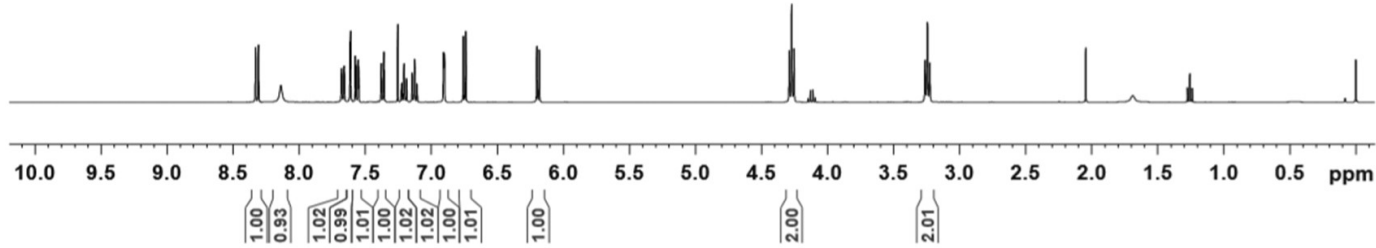

${ }^{13} \mathrm{C}$ NMR of $9\left(100 \mathrm{MHz}, \mathrm{CDCl}_{3}\right)$
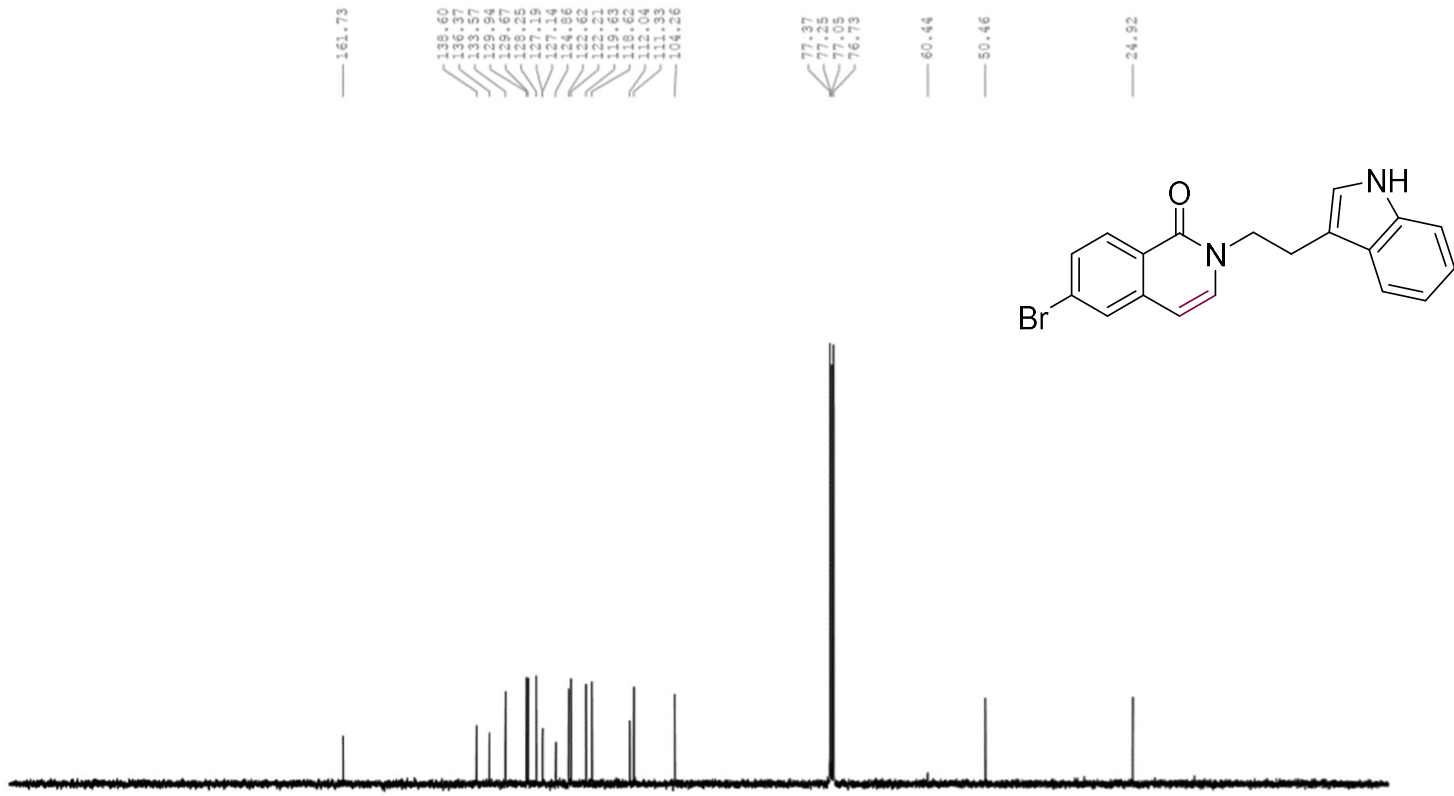

$\begin{array}{lllllllllllllllllllllll}210 & 200 & 190 & 180 & 170 & 160 & 150 & 140 & 130 & 120 & 110 & 100 & 90 & 80 & 70 & 60 & 50 & 40 & 30 & 20 & 10 & 0 & \mathrm{ppm}\end{array}$ 
${ }^{1} \mathrm{H}$ NMR of 10 (400 MHz, DMSO- $d_{6}$ )

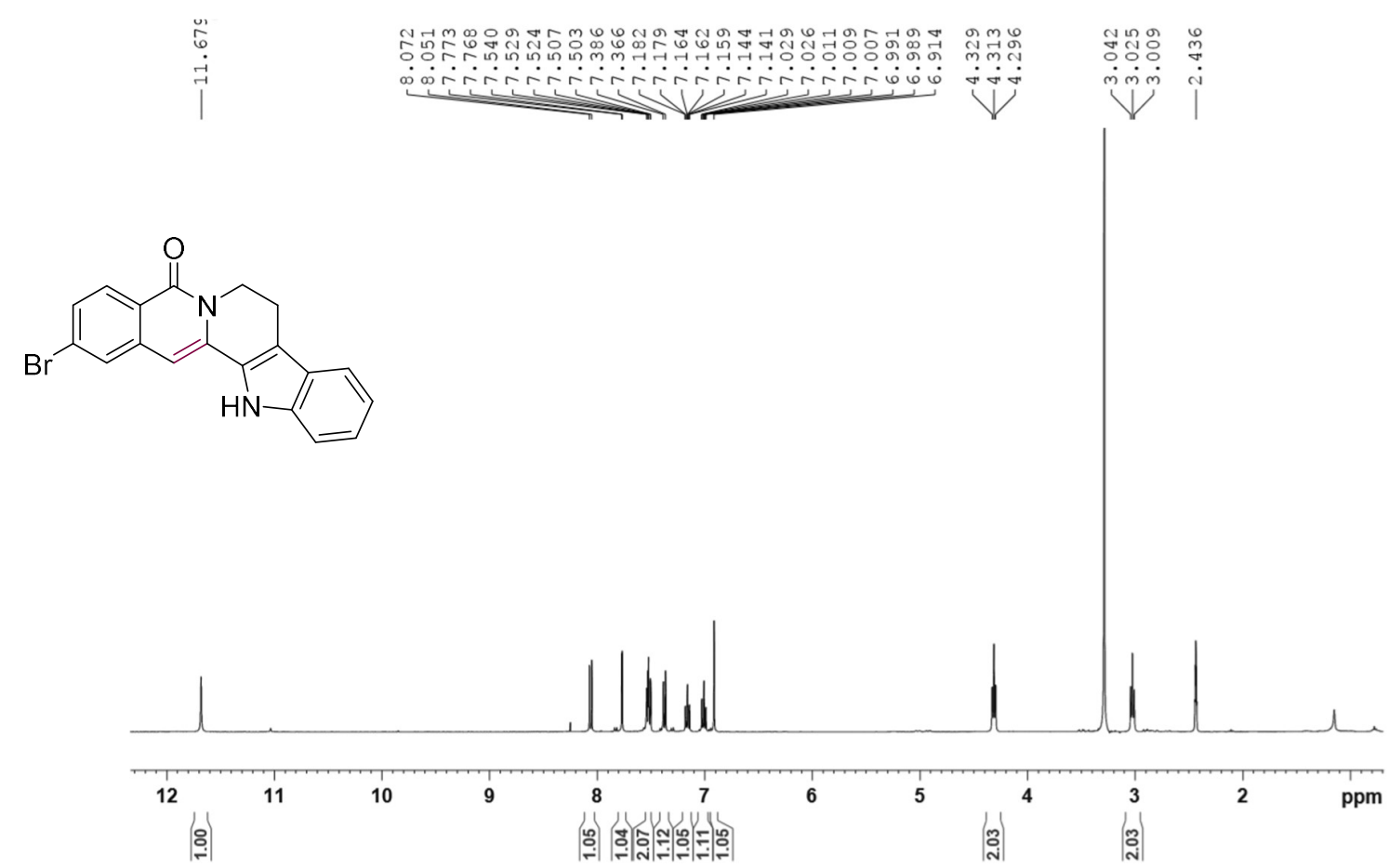

${ }^{13} \mathrm{C}$ NMR of $3 \mathbf{e}\left(100 \mathrm{MHz}, \mathrm{DMSO}-d_{6}\right)$

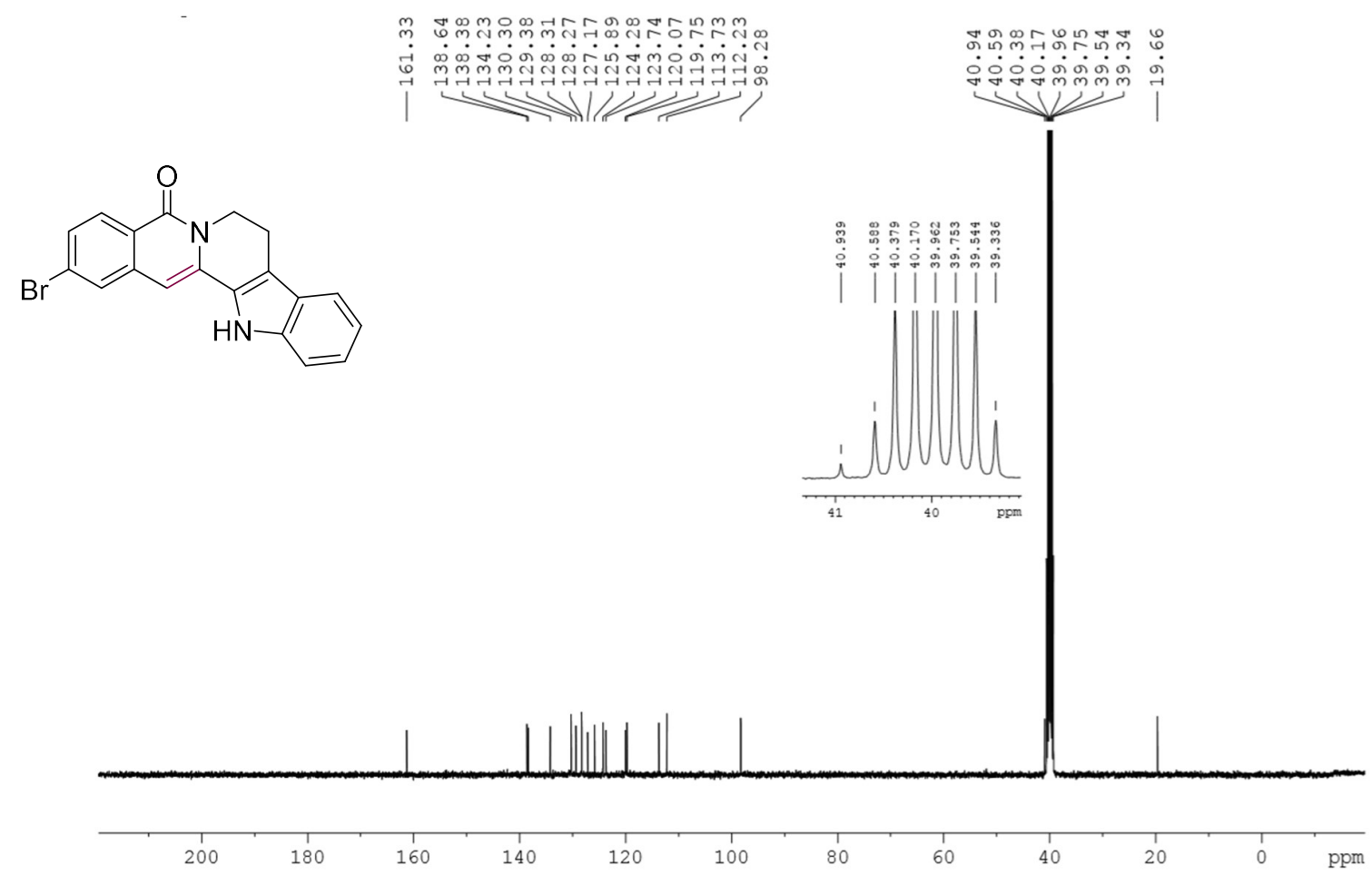




\section{References}

Santhi, J.; Baire, B. Chem. Asian J. 2019, 14, 3161-3165.

Izumi, T.; Nishimoto, Y.; Kohei, K.; Kasahara, A. J. Heterocyclic Chem. 1990, 27, 1419-1424.

3 Mallampudi, N. A.; Reddy, G. S.; Maity, S.; Mohapatra, D. K. Org. Lett. 2017, 19, 8, 2074-2077.

${ }^{4}$ Belgaonkar, V. H.; Usgaonkar, R. N. J. Heterocyclic Chem. 1978, 15, 257-260.

Mal, D.; Bandyopadhyay, M.; Ghorai, S. K.; Datta, K. Tetrahedron Lett. 2000, 41, 3677-3680.

${ }^{6}$ Yang, Y.; Buchwald, S. L. Angew. Chem., Int. Ed. 2014, 53, 8677-8681.

${ }^{7}$ Sheldrick, G. M. Acta Crystallogr. Sect. A 2008, 64, 112-122.

${ }^{8}$ Farrugia, L. J. J. Appl. Cryst. 2012, 45, 849-854.

${ }^{9}$ Frisch, M. J.; Trucks, G. W.; Schlegel, H. B.; Scuseria, G. E.; Robb, M. A.; Cheeseman, J. R.; Scalmani, G.; Barone, V.; Petersson, G. A.; Nakatsuji, H.; Li, X.; Caricato, M.; Marenich, A. V.; Bloino, J.; Janesko, B. G.; Gomperts, R.; Mennucci, B.; Hratchian, H. P.; Ortiz, J. V.; Izmaylov, A. F.; Sonnenberg, J. L.; Williams-Young, D.; Ding, F.; Lipparini, F.; Egidi, F.; Goings, J.; Peng, B.; Petrone, A.; Henderson, T.; Ranasinghe, D.; Zakrzewski, V. G.; Gao, J.; Rega, N.; Zheng, G.; Liang, W.; Hada, M.; Ehara, M.; Toyota, K.; Fukuda, R.; Hasegawa, J.; Ishida, M.; Nakajima, T.; Honda, Y.; Kitao, O.; Nakai, H.; Vreven, T.; Throssell, K.; Montgomery, J. A., Jr.; Peralta, J. E.; Ogliaro, F.; Bearpark, M. J.; Heyd, J. J.; Brothers, E. N.; Kudin, K. N.; Staroverov, V. N.; Keith, T. A.; Kobayashi, R.; Normand, J.; Raghavachari, K.; Rendell, A. P.; Burant, J. C.; Iyengar, S. S.; Tomasi, J.; Cossi, M.; Millam, J. M.; Klene, M.; Adamo, C.; Cammi, R.; Ochterski, J. W.; Martin, R. L.; Morokuma, K.; Farkas, O.; Foresman, J. B.; Fox, D. J. Gaussian, Inc., Wallingford CT, 2016.

${ }^{10}$ Chai, J.-D.; Head-Gordon, M. Phys. Chem. Chem. Phys. 2008, 10, 6615-6620.

${ }^{11}$ Ehlers, A. W.; Böhme, M.; Dapprich, S.; Gobbi, A.; Höllwarth, A.; Jonas, V.; Köhler, K. F.; Stegmann, R.; Veldkamp, A.; Frenking, G. Chem. Phys. Lett. 1993, 208, 111-114. 